\title{
GRENZEN UND CHANCEN BEI DER ERSCHLIESSUNG DES ÄLTEREN ÄGYPTISCH
}

\section{WOLFGANG SCHENKEL}

\section{OFFENE FRAGEN}

UNSERE KENNTNIS DES ÄGYPTISCHEN, auch und gerade des älteren Ägyptisch, ist unsicher. Sie ist unsicherer noch, als dies die andauernden Kontroversen unter den Ägyptologen erkennen lassen. Auch vieles könnte man wissen wollen, was noch gar nicht als Frage aufgeworfen ist. Wer oder was aber ist schuld an unseren problematischen Kenntnissen und an unserer eingeschränkten Sicht auf die in tiefer Vergangenheit liegenden Realitäten?

\subsection{Die Alten Ägypter sind schuld}

Erstens: Die Menge der überlieferten Sprachzeugnisse steht in einem ausgesprochen schlechten Verhältnis zur Menge der schriftsprachlichen Verlautbarungen der Alten Ägypter, nicht zu reden von der gesprochenen Sprache. Fast alles, was je geschrieben wurde, ist verlorengegangen, auf den Müllhaufen geworfen oder, ohne besondere Veranlassung, auf die eine oder andere Weise im Fruchtland verlorengegangen. Erhalten blieb nur einiges, was aus unterschiedlichen Gründen in die Wüste gelangte oder in der Wüste angefertigt wurde oder, in der älteren Zeit recht wenig, im Fruchtland in feuchtigkeitsresistenten Stein gemeißelt wurde. Davon, welche Unmengen schriftlicher Verlautbarungen verlorengegangen sein müssen, zeugen nur zu deutlich die Hinterlassenschaften der Verwaltung in der Wüste, namentlich die Abüșìr-Papyri und das alLāhün-Archiv, die eine Vorstellung davon geben, in welchem Umfang aktenmäßig registriert und per schriftlicher Korrespondenz verhandelt wurde. Eine Vorstellung davon, welche Unmengen an Verlautbarungen auf Papyrus selbst hier verlorengegangen sind, geben Berechnungen Georges Poseners, die Joachim Quack zitiert, der sich selbst vor allem mit der Lückenhaftigkeit der Überlieferung literarischer Texte beschäftigt. ${ }^{1} \mathrm{Zu}$ den großen religiösen Textkorpora fehlen die Schreibstuben und Archive, in denen die Vorlagen der überlieferten Pyramiden- und Sargtexte und die auf Vorrat geschriebenen, nie jedoch für den vorgesehenen Zweck genutzten Texte erstellt und abgelegt wurden. Es fehlen die ersten Niederschriften wissenschaftlicher Texte, so eines Teils der medizinischen Texte, die im Alten Reich verfasst worden sein müssen, namentlich die des medizinischen Papyrus Smith. Sehr vieles ist in den überlieferten Texten zu schwach belegt, als dass man sich ein fundiertes Urteil erlauben könnte, z.B. über die einzelnen Lexeme und ihre Formenbildung. Das alles ist dem Ägyptologen geläufig. Man hat aber eine Tendenz dazu, das Überlieferte als Informationsquelle einfach hinzunehmen, ohne allzu sehr darüber nachzudenken, was einem fehlen könnte. Etwas mehr Zurückhaltung sollte nicht schaden!

Zweitens: Die Qualität der überlieferten Sprachzeugnisse ist teilweise recht mangelhaft. Die Texte sind nicht immer mit der uns wünschenswerten Sorgfalt verfasst oder abgeschrieben worden, im Allgemeinen nicht „korrekturgelesen“. Sie sind also nicht selten unklar und fehlerhaft, was beides den Philologen oder Linguisten auf die falsche Fährte bringen kann. Es ist bei Weitem nicht alles, was geschrieben steht, korrektes Ägyptisch. Es sei beispielsweise erinnert an Fehler beim Zeilenwechsel, vor allem aber an fehlerhafte Auflösungen gespaltener Kolumnenschreibungen. Recht gut lässt sich das an Texten ablesen, die mehrfach überliefert sind. Abweichungen zwischen den Versionen beruhen gewiss zum Teil auf sinnvoller Variation eines Grundtextes, zum Teil aber auch auf Gedankenlosigkeit und mangelnder 
Konzentration beim Abschreiben einer Vorlage, wenn nicht schon bei der Niederschrift des Urtextes. Leicht zu erkennen sind Fehler auch in formelhaften Wendungen, wie sie etwa in Briefen auftreten. Überfordert ist hier nicht selten der Linguist, es bedarf des Einsatzes des Philologen.

Drittens: Ein Teil der Texte ist schwer verständlich, weil nicht-realweltliche Dinge behandelnd, die uns nicht unmittelbar aus eigener Erfahrung zugänglich sind, so allzu oft die Pyramiden- und Sargtexte, ein anderer Teil deshalb, weil er zu anspruchsvoll formuliert ist, die Kenntnis eines Wortschatzes und einer Idiomatik voraussetzt, die uns nicht ohne Weiteres zu Gebote steht, so z.B. bei den rhetorisch abgehobenen Reden im Beredten Bauern.

Viertens: Die Hieroglyphenschrift, wunderbar geeignet für den, der Ägyptisch kann, unterschlägt einen guten Teil der lautlichen Realität, die der Philologe und noch mehr der Linguist benötigt. Es fehlen weitestgehend die Vokale oder auch schon Indizien für die Position der Vokale innerhalb der Konsonantenfolge. Infolgedessen streitet man sich heute um die Formenbildung. Die unglücklichste Entscheidung, die man hier treffen kann und die tatsächlich von manchen fallweise auch explizit getroffen wurde, ist die positivistische Annahme, dass, was in der Schrift nicht unterschieden wird, auch in der Realität nicht unterschieden war. Es sei beispielsweise auf die unterschiedlichen Antworten auf die Frage hingewiesen, wie viele Verbalformen, ,śç $m=f$ "voneinander zu unterscheiden sind, eine Frage, auf die unten noch einzugehen sein wird. Gelegentlich kann zweifelhaft sein, ob ein Phonogramm als Notation eines Konsonanten oder als Komplement zu einer vorangehenden Notation dient. So kann, um ein folgenreiches Beispiel zu zitieren, zweifelhaft sein, ob nachfolgendem Einkonsonantenzeichen $n$ geschrieben ist, als $m=k$ in.tw „Sieh/Also, es wird gebracht“ mit Komplement $n$ zu lesen ist oder als $m=k$ in.n.tw „Sieh/Also, es wurde gebracht“, ,Sieh/Also, dass gebracht wurde" mit einem zweiten $n .^{2}$ Es stellt sich hier nämlich die generelle Frage, ob oder in welchen Fällen nach $m=k$,sieh/also" eine prädikative Verbalform stehen kann oder eine abstrakt-relativische stehen muss, eine Frage, die in der Abrechnung der neueren Linguistik mit der an Polotsky anschließenden Standardtheorie übersehen wurde. ${ }^{3}$ Ein anderes Problem: Es fehlt, von Verspunkten einmal abgesehen, eine Interpunktion, an der man die syntaktische Struktur ablesen könnte. Wie sehr man hier heute in die Irre gehen kann, zeigt der Vergleich der ägyptologischen Übersetzungen untereinander, die sich im Einzelnen nicht selten widersprechen. Was der eine gedanklich miteinander verbindet, kann ein anderer voneinander trennen wollen. Dazu später Beispiele.

Natürlich waren die Ägypter nicht verpflichtet, dem heutigen Philologen oder Linguisten zuzuarbeiten. Von Schuld kann also allenfalls im Sinne von Ursache die Rede sein. Dann also:

\subsection{Die Ägyptologen sind schuld}

Erstens: Die ägyptologische Sprachforschung bewegt sich zwischen Philologie und Linguistik. Keiner der Zugänge, weder der philologische noch der linguistische, ist für sich allein ausreichend. Der Philologie bedarf man, um einen sachgemäßen Zugang zu den Textquellen zu gewinnen, der Linguistik, um die richtigen Fragen zu stellen. Keiner der Zugänge, weder die philologische Aufbereitung des Materials noch die linguistische Einordnung der Befunde, ist zu einem zufriedenstellenden Abschluss gekommen. Im

2 Schenkel 2012c. (Die Entscheidung für die abstrakt-relativische Verbalform ist im gegebenen Fall korrekt, die Regel dagegen, derzufolge nach $m=k$ ausschließlich eine abstrakt-relativische Verbalform stehen kann, ist, worauf Helmut Satzinger (2013, 308) hinweist, zu modifizieren; dem trägt im gegenwärtigen Zusammenhang behelfsmäßig die Erweiterung von „ob“ durch „oder in welchen Fällen“ Rechnung.

3 Aufschlussreich die Diskussion in Collier 1992. 
Übrigen fehlt bei den Ägyptologen nicht selten die Balance zwischen den beiden Polen. Mancher klebt an den Texten, ohne sich allzu sehr um eine linguistische Basis für die Beurteilung der Befunde zu kümmern; mancher projiziert allgemein-linguistische Fragestellungen in das Textmaterial hinein, ohne sich der Tücken der Materialbasis bewusst zu sein.

Zu letzterem Fall ein kleines Beispiel: a Arme ( $\Omega$ ) kann die Negation $n$ geschrieben sein, aber auch die Fragepartikel (i) $n$, was letztlich mit der banalen Ähnlichkeit der Hieroglyphe mit dem Einkonsonantenzeichen $n(-m)$ im Hieratischen zusammenhängt. An der folgenden Stelle der Sinuhe-Erzählung kann man schwanken, ob in der Handschrift B die Hieroglyphe als die Negation oder als die Fragepartikel zu lesen ist: ${ }^{4}$

nink $\operatorname{tr} \operatorname{sm} 3(. w)=f \ldots$

I am not his ally, $\ldots{ }^{5}$

oder

(i)n ink tr $\operatorname{sm} 3(. w)=f \ldots$

So am I some ally of his, .... $?^{6}$

Hier hilft ein Blick auf die andere Handschrift, AOS, und auf das allgemeine Verhalten der Handschriften der Sinuhe-Erzählung weiter: Handschrift AOS hat in partikel, nämlich in-iw, und auch an einer anderen Textstelle findet sich dem entsprechend in der Handschrift B (i)n, in der Handschrift AOS $i n-i w^{7}$ Die unreflektierte Lesung der Hieroglyphe in Handschrift B als Negation datiert offenkundig in eine Zeit zurück, als die Handschrift AOS noch nicht bekannt war.

Zweitens: Die Ägyptologie, selbst die Linguistik-ferne, nutzt hochentwickelte Instrumente, die ältere und andere Philologien entwickelt haben, nur partiell oder erst neuerdings ansatzweise. Das jedenfalls gilt für die Textkritik, namentlich für die stemmatische Einordnung der mehrfach überlieferten Texte. Von speziellem Interesse für den Ägyptologen, zumal den linguistisch orientierten, ist dabei nicht die Rekonstruktion eines - „fehlerfreien“ - Urtexts, sondern die Möglichkeit, aus dem wechselseitigen Verhältnis der Textzeugen auf deren Qualität zu schließen. Es lassen sich Irrtümer der Schreiber erkennen, die den Linguisten auf eine falsche Fährte führen können. Z.B. wurden die Texte für bestimmte Anwendungen durch mechanische Austauschoperationen adaptiert, die fallweise zu sprachlich fehlerhaften Sequenzen führten, so bei der Ersetzung eines auf den Grabinhaber zu beziehenden Personalpronomens durch Titel und Eigennamen desselben. Der Ägyptologe erkennt den Fehler ohne weiteres, wenn dadurch eine syntaktisch falsche Wortfolge entstanden ist, wenn etwa das Subjekt einer Verbalform der Suffixkonjugation an die falsche Stelle geraten ist, wenn z.B. aus einer Sequenz Verbalform der Suffixkonjugation + Präposition $n+$ Suffixpronomen + Objekt die falsche Sequenz Verbalform der Suffixkonjugation + Präposition $n+$ Substantiv + Objekt geworden ist. Er kann aber auch auf die falsche Spur geleitet werden, wenn die Wortfolge syntaktisch einwandfrei bleibt, aber der Sinn des Textes beeinträchtigt ist, so, wenn gedankenlos ein satzinitiales Personalpronomen durch Titel und Eigenname ersetzt wird, ohne zu bedenken, dass fallweise diese Ersetzung korrekt ist, fallweise jedoch vor Titel und 
Eigenname die Partikel in zuzufügen gewesen wäre. Handelte es sich um einen Substantivalsatz, war die einfache Ersetzung korrekt; handelte es sich um das Satzmuster in-Konstruktion, wäre selbstverständlich in zuzufügen gewesen. ${ }^{8}$

Drittens: Bei manchen Entscheidungen spielen grammatische Vorurteile eine Rolle und Erwartungen zur Gewichtung der Teilaussagen im Zusammenhang der Textentwicklung. Das Fehlen einer Interpunktion erschwert nicht selten die Unterscheidung zwischen Hauptsatz und Nebensatz. Man kann sich darüber streiten, ob ein Adverbialsatz mit der Partikel $i w$ als Hauptsatz oder als einem vorangehenden Hauptsatz untergeordneter Nebensatz zu interpretieren ist. ${ }^{9} \mathrm{Z}$. B. gilt dies für eine oft übersetzte und auch von Grammatikern besprochene Satzfolge der Sinuhe-Erzählung wie die folgende: ${ }^{10}$

$n t f t$ ţr h $3 \hat{s} s .(w) t$ (Komma? Punkt?)

iw $i t=f m-\underline{h} n \cdot w r h=f$

śmi $=f \check{s} 3 . t n=f h p r(. w)$

Arbeitsübersetzung:

Er (Sesostris I., der Sohn) ist es, der die Fremdländer unterwirft.

Sein Vater (Amenemhet I.) ist in seiner Residenz.

Er (der Sohn) meldet, dass, was er (der Vater) angeordnet hat, ausgeführt ist.

Die Interpreten gliedern fast alle die Satzfolge so, als ob nach dem ersten der drei Sätze ein Komma stünde: ${ }^{11}$

Er (Sesostris I., der Sohn) ist es, der die Fremdländer unterwirft, (Komma)

während sein Vater (Amenemhet I.) in seiner Residenz ist,

und (wörtl.: ,indem“) er (der Sohn) meldet, dass, was er (der Vater) angeordnet hat, ausgeführt ist.

Einzelne aber gliedern so, als ob ein Punkt stünde: ${ }^{12}$

Er (Sesostris I., der Sohn) ist es, der die Fremdländer unterwirft. (Punkt)

Sein Vater (Amenemhet I.) (aber) ist in seiner Residenz,

und (wörtl.: ,indem“) er (der Sohn) meldet, dass, was er (der Vater) angeordnet hat, ausgeführt ist.

Die Frage ist von sprachgeschichtlichem Interesse. ${ }^{13}$ Ohne jeden Zweifel handelt es sich bei solchen Satzfolgen im Alten-Reichs-Ägyptisch um zwei aufeinander folgende Hauptsätze, im jüngeren Ägyptisch dagegen um einen Hauptsatz und einen Nebensatz. Wie aber sind Satzfolgen zu verstehen, die in Texten einer mutmaßlichen Übergangszeit stehen, also z.B. in der Sinuhe-Erzählung? U.a. stellt sich hier die Frage, wie die Textstellen im weiteren Zusammenhang eines Textes zu interpretieren sind. Wird mit einem $i w$ Hauptsatz ein Begleitumstand herausgehoben, oder wird mit einem $i w$-Nebensatz beiläufig ein Nebenumstand benannt? Hier ist der Philologe gefordert.

Wie unsicher aber auch Philologen sein können, lässt sich an Übersetzungen altbekannter literarischer Texte ablesen, z.B., willkürlich herausgegriffen, an der Übersetzung einer Stelle der Sinuhe-Erzählung, an der vom Passieren der Grenzfestung Sile berichtet wird. ${ }^{14}$ Ein guter Teil der Übersetzer versteht den

8 S. die Diskussion bei Schenkel 2012a, 143 (§ 6.1.1.2).

9 Loprieno 2006, Schenkel 2007a.

10 Sin. B 50-51.

11 Eine kleine Auswahl der einschlägigen Übersetzungen ist zitiert bei Schenkel 2007a, 171-73.

12 So jedenfalls Junge 1979, 268, Parkinson 1997, 31, und diesen zustimmend Schenkel 2007a, 171-73. S. auch in diesem Sinne, Stauder 2013,459 u. 502 fn. 219.

13 Ausführlicher hierzu Schenkel 2007a.

14 Sin. B 15-19. 
Erzählgang als eine etappenweise Beschreibung der Flucht des Sinuhe. Berichtet würde hier also, dass Sinuhe die Grenzfestung Sile erreicht. Um mich hier nicht in Übersetzungsvarianten zu verlieren und auch nicht einen bestimmten Übersetzer an den Pranger zu stellen, biete ich den Text in Anlehnung an die Handschrift B in eigener Verantwortung:

$(r c ̧ .,\{t\}<n>=i$ w.t $n$ rt. $. y(i)=i m h t)$

tmi. $n=i$ inb(.w) hlk ir. iry $r$ hśf śt. (i)w (Punkt)

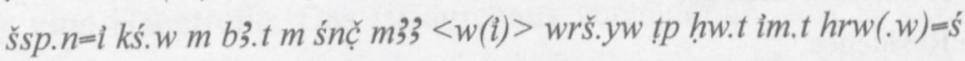

(Nachdem ich den Weg nach Norden eingeschlagen hatte,)

erreichte ich die Festung des Herrschers, die gemacht war, um die Syrer abzuhalten. (Punkt)

Ich kauerte mich in ein Gebüsch aus Furcht, es könnte mich entdecken die Wachmannschaft auf der Mauer, die Tagesdienst hatte.

Für andere Übersetzer handelt es sich bei der Aussage über die Festung um einen Umstand, der Sinuhe dazu zwang, sich bis zum Eintritt der Dunkelheit zu verstecken, um im Schutze der Nacht die Grenze unbeobachtet überschreiten zu können:

$($ rç.t $t=i$ w3.t $n$ rț.y(i)=i $m$ ht $)$

tmi.n=i inb(.w) hlk ir.y $r$ hśf śt. (i) $w$ (kein Punkt)

šsp.n=i kś. $w$ m b3.t $m$ śnç $m 3 \xi<w(i)>$ wrš. yw tp ḥw.t im.t hrw(.w)=ś

(Ich schlug den Weg nach Norden ein.)

Als ich die Festung des Herrschers erreicht hatte, die gemacht war, um die Syrer abzuhalten, (kein Punkt)

kauerte ich mich in ein Gebüsch aus Furcht, es könnte mich entdecken die Wachmannschaft auf der Mauer, die Tagesdienst hatte.

In diesem Fall läge, den Übersetzern als linguistisches Faktum kaum bewusst, das von Pascal Vernus entdeckte zweite Schema der Emphatischen Konstruktion, meine Rang-V-Erweiterung, vor (oder wie immer man den grammatischen Sachverhalt formulieren mag). Was an der Textstelle gemeint sein könnte, lässt sich entscheiden, wenn man die Textstelle im weiteren Zusammenhang der Erzählung sieht: Anders als ein guter Teil der Philologen, die sich besonders für die Fluchtroute interessieren, interessieren den Erzähler eher die Gefahren, denen der Held ausgeliefert ist. Wie dem auch sei: Es sollte abschließend der Philologe dann doch explizit überprüfen, ob seine Interpretation auch unter grammatischen Gesichtspunkten vertretbar ist. Im gegebenen Fall steht zu vermuten, dass die Übersetzer, die die m.E. richtige Lösung gefunden haben, keine rechte Vorstellung von der dazu passenden syntaktischen Struktur hatten, dass diese Lösung unter grammatischem Gesichtspunkt überhaupt möglich ist. Ältere Übersetzer hätten in keiner Grammatik die passende Erklärung finden können, jüngere aber hätten Vernus gelesen haben sollen. ${ }^{15}$ Es ist dies übrigens nicht das erste Mal, dass Gebüsch als Ziel einer Absetzbewegung für Sinuhe von essentieller Bedeutung ist. Bereits bei der panikartigen Flucht aus der Truppe endet die Suche nach einer Stelle, an der er sich verbergen könnte, zwischen zwei Büschen. ${ }^{16}$

Als eine weitere Textstelle der Sinuhe-Erzählung, deren Diskussion möglicherweise zu weit in Details gehen würde, sei noch die folgende erwähnt: ${ }^{17}$ 


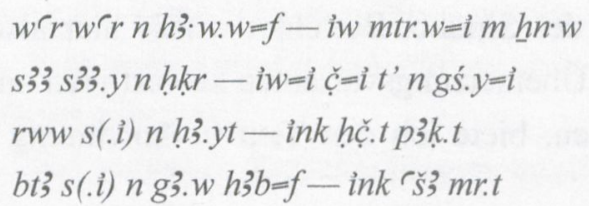

Ein Literaturwissenschaftler fügt, inhaltlich nachvollziehbar, vor dem jeweils zweiten Satz ein „but“" ein: ${ }^{18}$

A fugitive takes flight because of his surroundings;

but my reputation is in the Residence.

A creeping man creeps off because of hunger;

but I give bread to my neighbour.

A man leaves his land because of nakedness;

but I am plentiful of serfs.

Ein Linguist dagegen setzt „,but“ nur, wenn der zweite Satz nicht mit iw, sondern mit ink beginnt: ${ }^{19}$

Due to his situation, a fugitive flees; my reputation is in the residence.

Due to hunger, a wanderer lingers; I give bread to my neighbour.

Due to nakedness, a man leaves his land; but mine is the fine linen.

[Das vierte Satzpaar, das auf der nächsten Seite der Edition folgt, ist übersehen worden.]

Erkennbar hat der Linguist die syntaktische Struktur im Auge: Der jeweils erste Satz ist als Emphatische Konstruktion eingeschätzt. Aber wie verhält sich der jeweils zweite Satz zum jeweils ersten?

Ein weiterer, willkürlich herausgegriffener Übersetzer macht aus dem jeweils ersten Satz einen Nebensatz: $:^{20}$

alors que c'est à cause de son entourage qu'un fugitif fuit, je suis illustre au pays.

Alors que c'est à cause de la faim qu'un vagabond vagabonde, je donne du pain à mon voisin.

Alors que c'est par dénuement qu'un homme fuit son pays, à moi du linge blanc et le tissu fin !

Alors que c'est faute de quelqu'un à envoyer à sa place qu'un homme court, j'ai de nombreux serviteurs !

Man kann den Eindruck haben, dass hier der jeweils erste Satz als Emphatische Konstruktion verstanden ist, der Satz aber in Verbindung mit dem jeweils zweiten Satz als eine Rang-V-Erweiterung des letzteren fungiert. Eine solche Kombination hat $\mathrm{m}$.W. noch niemand für möglich gehalten. Vielleicht handelt es sich bei dem ,alors que“ lediglich um eine elegantere Verbindung der beiden Sätze, die andere Übersetzungen mit einem „but" vor dem jeweils zweiten Satz realisieren. Übrigens habe ich selbst, zu Recht oder Unrecht, den jeweils ersten Satz zu einer Rang-V-Erweiterung erklärt, dabei aber auf die Emphatische Konstruktion als eine - im Prinzip denkbare - Erklärung des jeweils ersten Satzes verzichtet. ${ }^{21}$

Viertens: Die Linguistiken kommen und gehen, sie kommen und gehen zu schnell, als dass die Ägyptologie folgen könnte. Die besten heute verfügbaren Grammatiken stehen noch weitgehend in der Tradition der Philologie und der Sprachwissenschaft des 19. Jahrhunderts. Alan H. Gardiner, der mit seiner

18 Parkinson 1997, 34.

19 Uljas 2008, 207.

20 Grandet 1998, 25.

21 Schenkel 2012a, $313(\S 8.3 .4 .2 .1), 315-16(\S 8.3 .4 .2 .2)$. 
Egyptian Grammar den größten Erfolg hatte, nahm den Strukturalismus, die zu seinen Lebzeiten einflussreichste zeitgenössische Linguistik, erst richtig wahr, als seine Grammatik bereits geschrieben war. Er hat sich dann auch, nebenbei bemerkt, selbst an der zeitgenössischen Linguistik-Diskussion beteiligt. ${ }^{22}$ Bemerkenswert ist dann aber noch, dass Gardiners Grammatik ihre größte Wirkung erst nach der 2. Auflage von 1950 entfaltete, zu einer Zeit also, in der der Strukturalismus sozusagen die Standardtheorie war.

In der neueren und auch noch in der aktuellen Diskussion spielt die Auseinandersetzung mit Hans Jakob Polotsky eine Hauptrolle. Das ist durchaus verständlich, war es doch Polotsky, der die Diskussion der ägyptischen Grammatik erst wieder richtig in Gang brachte, nachdem die Ägyptologenschaft größtenteils der Vorstellung huldigte, die Berliner Schule und ihre Anhänger anderswo hätten mit Grammatiken und Wörterbuch eine solide Basis geschaffen, auf der man sich jetzt sorglos höheren Gegenständen der altägyptischen Kultur zuwenden könne. Eine vergleichbare Situation lässt sich heute erneut beobachten: Ist nun abermals mit der Polotsky zugeschriebenen "Standardtheorie" ein gewisser Abschluss erreicht oder beginnt nicht jetzt erst eine neue Phase der linguistischen Arbeit? Polotsky selbst war nicht davon überzeugt, eine "Standardtheorie" entwickelt zu haben. Er sah Lücken und weiteren Forschungsbedarf.

Wer Polotsky bzw. die Standardtheorie überwinden will, will damit auch den Strukturalismus überwinden, die Syntaxorientierung der Polotskyschen Arbeiten. Polotsky selbst war kein enger Strukturalist. Er beherrschte auch die älteren Strömungen der allgemeinen Sprachwissenschaft, die historischvergleichende Sprachwissenschaft des 19. Jahrhunderts, und, vor allem: Er war auch Philologe. Er kannte seine Textquellen und konnte selbst daraus aussagekräftiges Belegmaterial gewinnen, verließ sich also, anders als so manch anderer, nicht auf die ägyptologische Sekundärliteratur oder auf Schulkenntnisse, was die Texte angeht. Wenn man Polotsky Strukturalismus vorwirft, sollte man mehr noch den zeitgenössischen allgemein-linguistischen Strukturalisten der 1940er und 1950er Jahre Schuld zuweisen. Denn diese waren es doch, die sich einseitig auf die Syntax konzentrierten und die Beschäftigung mit der heutzutage so dominierenden Semantik mit Entschiedenheit ablehnten.

Eine umfassende Darstellung der ägyptischen Grammatik unter strukturalistischen Vorzeichen kam nie zustande, obwohl doch der Strukturalismus Jahrzehnte lang weite Bereiche der Linguistik beherrschte. Mir fällt als Anlauf zu einer solchen Darstellung nur ein Beitrag von Carlton T. Hodge ein, der ein Gerippe der Grammatik nach Gardiners Egyptian Grammar in strukturalistische Terminologie übersetzt hat. ${ }^{23}$ Ganz anders übrigens die zeitgenössische Altorientalistik, die, näher am Puls der Zeit, noch in der Hochzeit des Strukturalismus eine strukturalistische Darstellung des Akkadischen zustande brachte. ${ }^{24}$ Ich will nicht näher eingehen auf Richtungen der Linguistik, die sich zu einer Zeit, als besonders die nordamerikanischen Strukturalisten die Semantik zum Tabu erklärten, mit Semantik beschäftigten, die Sapir-Whorf-Richtung in Nordamerika und die inhaltbezogene Sprachwissenschaft Leo Weisgerbers in Deutschland, der ich selbst einmal einige Anregungen verdankte.

In den 1960er Jahren machte die Generative Transformationsgrammatik Furore. Welche Spuren hat Noam Chomskys innovatives Konzept in der Ägyptologie hinterlassen? Mir fällt da am ehesten noch Friedrich Junges Skizze einer „Darstellung der Ägyptischen Grammatik als Satzgenerator“ im Anhang seiner „Syntax 
der mittelägyptischen Literatursprache“ von 1978 ein. ${ }^{25}$ Meinen eigenen Beitrag von 1968 zu den „Syntagmen mit infiniten Verbalformen als Transformate von Sätzen“ möchte ich nur der Ehrlichkeit halber erwähnen. ${ }^{26}$ Letzten Endes verlief die Beschäftigung mit der Generativen Transformationsgrammatik im Sande.

Seither ist in der Linguistik sehr viel passiert. Die neuere Linguistik hat bereits tiefe Spuren in der Ägyptologie hinterlassen. Anders als früher haben sich auch eine ganze Reihe von Ägyptologen auf das engere Gebiet einer ägyptologischen Linguistik spezialisiert. Es steht also zu erwarten, dass man besser als früher Konzepte der allgemeinen Linguistik aufgreifen und substantielle Ergebnisse erzielen kann. Dennoch bleibt Skepsis. Auch die Linguistik hat größeres Potential gewonnen und entwickelt sich heute sehr viel schneller als dies etwa in der Blütezeit des Strukturalismus oder gar der Blütezeit der historischvergleichenden Sprachwissenschaft der Fall war. Aber vor allem dies: Die ägyptischen Textquellen werden nicht besser und nicht viel ergiebiger, auch wenn man bessere Fragen an das Material richtet. Dass dies so ist, sieht man an dem derzeitigen Chaos - ich erinnere mit diesem Wort an Crossroad (I)! $!^{27}$ - in der ägyptologischen linguistischen Sekundärliteratur.

\subsection{Traditionalismus und Fortschrittsglaube}

Die Ägyptologen sind teils fortschrittlich gesonnen, teils traditionalistisch eingestellt, schließlich und das vor allem können Traditionalismus und Fortschrittsglaube unvermittelt nebeneinander stehen. Manche Differenzen sind ohne großen Belang. Aber könnte man sich nicht besser auf die großen Fragen konzentrieren, wenn man zuerst einmal die kleinen Fragen aus der Welt schaffen würde?

Erstens: Ein Bereich, in dem man sich eigentlich auf gemeinsame Lösungen verständigen könnte, sind Transkription und Transliteration („Transliteration“ im Sinne der linguistischen Terminologie, nicht im Sinne des anglophonen ägyptologischen Sprachgebrauchs). Unbedachter Traditionalismus bei ansonsten fortschrittlicher Grundeinstellung lässt sich namentlich bei der Transkription beobachten, ein Beharren auf Lautformen, die längst überholt sind. Z.B. sollte heutzutage klar sein, dass nach der Transkriptionsnorm,

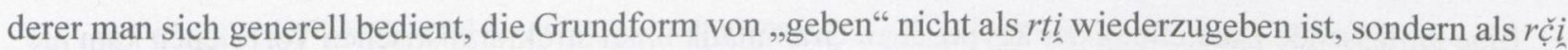
(NB: Es geht hier nicht um die Wahl der Transkriptionszeichen, sondern um den damit notierten Lautwert). „Fest“ ist nicht $h b$, sondern $h \xi b$, „,knüpfen“ ist nicht $\check{c} s$, sondern $c ̌ s s$. Grundsätzlich wird das ältere Ägyptisch entsprechend den älteren (nicht gerade den allerältesten) Graphien transkribiert, es sei denn eine Graphie lässt unbezweifelbar eine jüngere Lautform erkennen. Bleibt ein Konsonant ungeschrieben, wird er in Klammern ergänzt, z.B. in $h(3) b$. $h b$ wäre als Transliteration der altmodischen, aber visuell befriedigenden Zeichenanordnung in einem Schrift-"Quadrat" denkbar, ist als Transkription jedoch nicht akzeptabel. Die

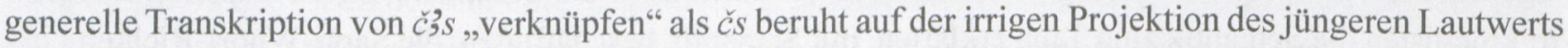
von $₫ \triangleleft$ in Graphien von $\check{c} s i$, ,erheben“ in das von diesem strikt zu unterscheidende $\check{c}$ ’s, erst jünger $\check{c} s$, „knüpfen“. Nur wenn an einer Flexionsform ablesbar ist, dass das Verb 2-radikalig geworden ist, sollte man diese Flexionsform selbst und evtl. andere Flexionsformen im weiteren Textzusammenhang als čs trans-

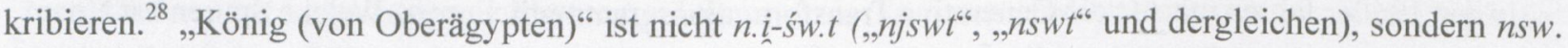
„Stadt" ist nicht niw.t, sondern $n$ '.t (man könnte sich, um ein eigenes Transkriptionszeichen für einen nicht

26 Schenkel 1968.

27 Englund und Frandsen 1986.

28 Andréas Stauder macht mich auf das reduplizierende passivische Partizip čss.t aufmerksam, das nur von einem 2-rad. Verb gebildet werden kann (zu Belegen bereits aus dem Mittleren Reichs s. Gardiner 1927/1950/1957, 277 n. 10). 
sicher $\mathrm{zu}$ bestimmenden Konsonanten $\mathrm{zu}$ umgehen, auf $n w . t$ einigen). ${ }^{29}$ Die paradigmatisch verankerte reduplizierte Form von rç̌i ,geben" ist nicht tiți, sondern ç̣̆ bzw. tț. Es gibt einen Konsens, dass es dreiund-mehr-konsonantige Phonogramme gibt, es gibt aber keinen Konsens darin, wieviele und welche Schriftzeichen als solche anzusetzen sind. ${ }^{30}$ Einige wenige Schriftzeichen lassen sich zwingend als solche Phonogramme bestimmen. ${ }^{31}$ Man sollte jedoch die Existenz der raren Dreikonsonantenzeichen nicht, der Communis opinio folgend, ausgerechnet mit exemplifizieren und nicht diesen Ansatz mit der Schreibung einer allenfalls hypothetischen drei-konsonantigen Endung begründen. ${ }^{32}$ - Solcherlei Notationen mögen aus der Perspektive eines auf andere Bereiche der Sprache konzentrierten Ägyptologen belanglos sein. Sie sind aber von Belang für Fragen der (etwa hamitosemitischen) Etymologie, der Kulturgeschichte und für die Rekonstruktion der verbalen bzw. nominalen Formenbildung.

Zweitens: Transkription und Transliteration (im Sinne der linguistischen Terminologie, nicht im Sinne des anglophonen ägyptologischen Sprachgebrauchs) stehen in einem Konkurrenzverhältnis: Transliteriert man hieroglyphische Schriftzeichen oder notiert man Lautformen, die der Ägypter mutmaßlich mit den hieroglyphischen Schriftzeichenfolgen notieren wollte? Im Allgemeinen wird transkribiert. Es gibt aber Fälle, in denen kurioserweise transliteriert wird, so im Fall der Notation eines mit $\langle\vec{c}\rangle$ geschriebenen $t$, z.B. des Passiv- $t w$ oder des Demonstrativums $t n$, die zu keiner Zeit anstelle des [t] ein [t] hatten. Die Sachlage ist genau umgekehrt: Das Schriftzeichen $\langle\vec{c}\rangle$ hat im Laufe der Zeit einen zweiten Lautwert $t$ angenommen, nachdem in einem Teil der mit $\langle\vec{c}\rangle$ geschriebenen Wortformen das ursprüngliche $[\mathrm{g}] \mathrm{zu}$ [t] geworden war. Sollte es im Einzelfall erforderlich sein, den Gebrauch von $\langle\vec{c}\rangle$ für $t$ zu notieren, darf man das nicht auf Transkriptionsebene tun. Man muss auf die Transliterationsebene wechseln, was man durchaus auch innerhalb einer Transkription tun könnte. Z.B. könnte man ein für $t$ gebrauchtes $\langle\vec{c}\rangle$ als $t_{2}$ notieren, das sich durch den Index $/ 2$ von einem als $t_{/ /}$zu verstehenden indexlos geschriebenen $t$ unterschiede. ${ }^{33}$ Es gibt Fälle, in denen transliterative Maßnahmen wichtiger sein könnten als in den banalen, gerade veranschaulichten: Relevant für die linguistische Interpretation können Semogramme sein, namentlich Determinative, die in der Standard-Transkription nicht erkennbar oder nicht notiert sind.

Drittens: Bei der Transkription des Ägyptischen bemühen sich die einen darum, möglichst bei einer der tradierten Lösungen zu bleiben, die anderen möchten der linguistischen Realität nahe kommen. Beides ist bedenklich. Traditionelle Lösungen gibt es mindestens zwei: die Transkription der Berliner Schule mit ihren „Dialekten“, die besonders in der Version der Gardiner-Grammatik verbreitet ist, und die Transkription nach Elmar Edels Altägyptischer Grammatik, die linguistisch orientierte Ansätze Fritz Hintzes aufgreift. ${ }^{34}$ Keines der Transkriptionssysteme ist ideal, aber es gibt bessere und schlechtere. Für besser halte ich die

30 S. Schenkel 2012b, 27-29.

31 Zum Verfahren eines Nachweises Schenkel 2012a, 34 (§ 3.1.1.2, Anm. 1 und Diskussion zu Anm. 1).

32 „Un des rares trilitères à fonctionner véritablement comme phonogramme est le signe $\mathbb{N}$, qui se lit tjw (tiw), notamment dans certaines terminaisons grammaticales" (Winand 2013, 38, Fußnote 1); zur Lesung speziell der hauptsächlich gemeinten (Nisba-)Endung s. Müller 2012, 307, mit Verweis auf Edel. NB: Angesichts der Unklarheiten der Pluralbildung transkribiert man den Plural in der Regel mit dem Singular abzüglich - falls vorhanden - einer Femininendung $\boldsymbol{t}$ plus einer - meist fiktiven - Pluralendung $w$. Selbstverständlich sollte man alle nur fiktiven Konsonanten in runde Klammern einschließen. Z.B. Nisbe im Singular mit Endung $t i$ oder $t i$, deren $i$ oder $i$ real existiert, folglich auch geschrieben werden kann, im Plural dagegen schematisch aus dem Singular übernommen wird, folglich in runde Klammern zu setzen ist: $t(i) w$, denkbar auch $t(i) w$.

$33 \mathrm{Zu}$ einem solchen Verfahren s. Schenkel 1983a, 1-170 (NB: terminologisch hier noch nicht zwischen Transkription und Transliteration unterschieden).

34 M.W. auf persönlichen Mitteilungen beruhend. 
älteren, da sie zum einen der graphematischen Realität näher stehen (erinnert sei zumal an Ermans Unterscheidung von $j(\neg \backslash)$ und $\ddot{i}$ (II) und an die Erklärung von $\underline{t}$ und $\underline{d}$ als typographische Notbehelfe), zum anderen der phonetischen Realität näher stehen dürften (erinnert sei an die Wiedergabe der $s$-Laute als $s$ und $s$ und an die Unentschiedenheit - Aleph oder Jod - bei der Wiedergabe des einfachen Schilfblatts $Q$ mit $i$ ). Noch problematischer ist die Aufbereitung der Transkription mit zusätzlichen Maßnahmen für die Linguisten (ich erinnere an die Verwendung von Transkriptionszeichen des internationalen phonetischen Alphabets oder die Markierung von Transkriptionszeichenfolgen mit ,[“" und ,,]", was suggeriert, dass man durchgängig die phonetische Realisierung der Phoneme kennte. Selbstverständlich kann man die phonetische Realisierung im Einzelfall diskutieren und sich dabei der linguistischen Notation bedienen, man sollte aber nicht schematisch Wortformen mit solchen Kennzeichnungen anreichern, da man schlicht und einfach die phonetische Realisierung nicht durchgängig kennt.

Viertens: Am heikelsten ist die Terminologie im Bereich der verbalen Morphologie, zumal beim Verbum (Tempus, Aspekt, Modus, ...). Hier sind sich weder die Traditionalisten untereinander einig noch die linguistisch orientierten Neuerer. ${ }^{35}$ Wenigstens sollte man sich in diesem Bereich weitgehend darauf einigen können, wieviele und welche Formenbildungen unter paradigmatischen Gesichtspunkten voneinander zu unterscheiden sind (namentlich bei den śç̌m $(. w / y)=f$ 's, wozu unten mehr), oder man sollte sagen, dass dies nicht in vollem Umfang möglich ist, und explizit begründen, warum dies nicht möglich ist.

\subsection{Vorausschau}

Im folgenden soll an ausgewählten Beispielen punktuell vor Augen geführt werden, wie kompliziert die sprachliche Realität war ( $(2)$ und wie wenig man in anderen ausgewählten Fällen mangels geeigneterer Indizien auf Zugänge verzichten kann, deren sich der heute ungeliebte Strukturalismus bediente, nämlich der Syntax und der Paradigmatik (§ 3).

\section{Die ReAlitÄt Hinter den GRAPHiEN}

Wie gut oder schlecht man sich ein Bild von der lautsprachlichen Realität ägyptischer Flexionsformen machen kann, davon kann man sich eine Vorstellung verschaffen, wenn man sich Flexionsformen betrachtet, die sich zum mindesten partiell vokalisieren lassen. Die Realität ist vielfältiger als die hieroglyphischen Graphien dies zu erkennen geben. Hierzu drei Belege.

\subsection{Infinitiv}

Von jedem Verb kann, belegt oder nicht belegt, mindestens ein Infinitiv gebildet werden. Die Bildungsweise der Infinitive ist jedoch nicht einheitlich. Das lässt sich bereits an den Graphien ablesen: Ein großer Teil der Verben hat einen Infinitiv mit einem finalen Konsonanten $t$, der nicht zu den Radikalen gehört, ein anderer großer Teil der Verben einen Infinitiv, der ein solches $t$ nicht aufweist; schließlich gibt es auch vereinzelt Verben, für die beide Bildungsweisen belegt sind. Andere Bildungsweisen sind marginal und werden unten noch gestreift. Da es sich bei der großen Masse der Infinitive mit zusätzlichem finalem $t$ um Verben mit schwachem dritten Radikal handelt (III.inf., z.B. ir.t zu iri "tun"), könnte man vermuten, dass dieses $t$ anstelle des schwachen Radikals steht und damit der Stamm auf die Länge der Infinitive der „Standard“-Verben, d.h. der starken dreiradikaligen Verben (3-rad., z.B. śçm zu śḉm „,hören“) gebracht wird. Dazu würde auch passen, dass Verben mit schwachem viertem Radikal, bei denen das zusätzliche $t$ geläufig ist (z.B. IV.inf. ḩmś.t zu ḥmśi , „sitzen“), den Infinitiv teilweise ohne dieses $t$ bilden (z.B. IV.inf. $m \xi w\{i\}^{?} \mathrm{zu}$ 
$m$ 3wi „neu sein“) und damit die kürzere Stamm-Länge der „Standard“-Verben erzielen. In dasselbe Schema würden auch die $s$-Kausativa der zweiradikaligen Verben passen, die mit dem zusätzlichen $t$ (z.B. Kaus. 2 rad. ś:mn.t zu ś: $m n$ „,dauern lassen“) dieselbe Länge wie die Infinitive der Kausativa zu den dreiradikaligen

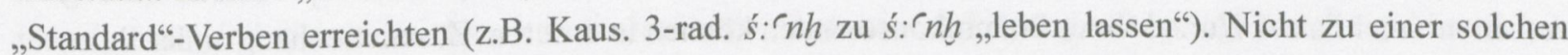
Erklärung passen die Infinitive der zweiradikaligen Verben, die ohne ein zusätzliches $t$ gebildet werden (z.B. 2-rad. $m n$ zu $m n$ „bleiben“). Da diese Verben keineswegs selten sind, könnte man sie als eine zweite Gruppe von „Standard“-Verben neben den dreiradikaligen „Standard“-Verben verstehen. Als „,irregulär“ blieben dann immer noch die bereits angeführten Verben mit schwachem viertem Radikal stehen, die trotz „ausreichender" Stammlänge das zusätzliche $t$ anhängen (z.B., bereits zitiert, IV.inf. hmś.t zu hmśri „sitzen“), und überhaupt Infinitive aus anderen, weniger prominenten Verbalklassen mit mehr als drei starken Radikalen (z.B. III.red. phrr zu phrr „laufen"). So weit Überlegungen allein auf der Basis der in den hieroglyphischen Graphien fassbaren Konsonanten.

Nun ist aber gerade für den Infinitiv über das in den hieroglyphischen Graphien fassbare konsonantische Gerippe hinaus auch die Vokalisation in großen Zügen rekonstruierbar. Bei Osing 1976a stellt sich die Lage so dar: ${ }^{36}$

(1) Bei den Infinitiven mit 3 Wurzel- bzw. Stamm-Konsonanten (II.red., 3-rad., III.inf.) ohne - der Normalfall — zusätzliche Endung sind vier Typen zu unterscheiden, die bei den starken dreiradikaligen Verben zu rekonstruieren sind als (1) *śáạcam, (2) *śaçắm, (3) *śśçčim und (4) *śiçím.

(2) Der Typ des Infinitivs hängt zum mindesten teilweise mit semantischen Faktoren zusammen:

(2.1) Infinitive des Typs (1), *śáç̌am, bilden in der Regel fientische Verben, nicht aber Zustandsverben; belegt sind Verben II.red., 3-rad. und III.inf. ${ }^{37}$

(2.2) Infinitive des Typs (2), *śaçắm, bilden vor allem Zustandsverben und intransitive fientische Verben; belegt sind Verben II.red., 3-rad. und III.inf. ${ }^{38}$

(2.3) Infinitive des Typs (3), *śšçčim, finden sich bei 3-rad. transitiven und intransitiven fientischen Verben sowie bei Zustandsverben, (häufig) bei III.inf. transitiven Verben, (weniger häufig) bei intransitiven fientischen Verben und (selten) bei III.inf. Zustandsverben. ${ }^{39}$

(2.4) Infinitive des Typs (4), *śiçútm, finden sich bei 3-rad. transitiven und intransitiven fientischen Verben sowie bei Zustandsverben, bei III.inf. bei Zustandsverben und (sporadisch) bei intransitiven fientischen Verben. ${ }^{40}$

Klar dürfte sein, dass semantische Faktoren eine Rolle spielen, nicht also nur die formale "Verbalklasse". Besonders klar liegen die Verhältnisse bei den Infinitiven mit $a$-Vokalisation, weniger klar bei den Infinitiven mit $i$-Vokalisation. Es ist hier nicht der Ort, die Einzelheiten zu diskutieren, die ohne Zweifel einer Diskussion bedürfen (ein Teil der Osingschen Belege ist möglicherweise problematisch, zumal die als „spät" gekennzeichneten; bei einem Teil der Belege, die erst „kopt.“ fassbar sind, könnte es sich im früheren Ägyptisch um Substantive gehandelt haben, ähnlich wie bei den unten unter Punkt (6) anzuführenden Infinitiven der IV.inf. auf -yt). Es genügt in unserem Zusammenhang die Feststellung, dass die Semantik nicht außer Betracht bleiben darf und dass dafür die Diskussion der Vokalisation die Voraussetzung ist. 
(3) Bei den Verben mit 4 Wurzel- bzw. Stammkonsonanten (III.red., 4-rad., IV.inf.) liegen teilweise ähnliche Beobachtungen wie bei den Verben mit 3 Wurzel- bzw. Stammkonsonanten vor. Wie letztere sind sie

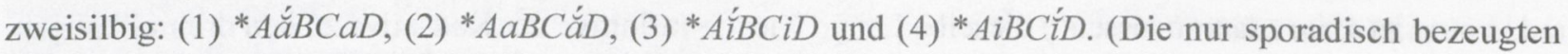
Verben mit mehr als 4 Wurzel- bzw. Stammkonsonanten können in unserem Zusammenhang außer Betracht bleiben.) Im Einzelnen:

(3.1) Infinitive des Typs (1), *AăBCaD, bilden häufig fientische Verben, dagegen selten Zustandsverben; belegt sind 4-rad. und IV.inf. Verben. ${ }^{41}$

(3.2) Infinitive des Typs (2), *AaBCắD, sind belegt für Kausativa zu Verben mit 3 Wurzelkonsonanten (NB: nicht Zustandsverben wie bei (2.2)). ${ }^{42}$

(3.3) Infinitive des Typs (3), *A (3.4) Infinitive des Typs (4), * $A i B C \breve{L} D$, sind zu schwach nachgewiesen, als dass sie für die vorliegenden Zwecke von Belang wären. ${ }^{44}$

(4) Bei Verben mit 2 Wurzel- bzw. Stamm-Konsonanten (2-rad.) steht zwischen den beiden Konsonanten ein Langvokal: *Aáa, *Aís. Vorausgesetzt, wie in der zitierten Sekundärliteratur angenommen, das Ägyptische unterscheidet zwischen Lang- und Kurzvokalen und nicht, wie in neuerer Zeit von anderen angenommen, offene und geschlossene Vokale; unter dieser Voraussetzung könnte man die Längung des Vokals als Kompensation interpretieren, als äquivalent mit den zwei Kurzvokalen der Verben mit 3 Wurzelbzw. Stamm-Konsonanten und der zwei Kurzvokale eines Teils der Verben mit 4 Wurzel- bzw. Stammkonsonanten. Eine solche Interpretation ist jedoch auch dann zu problematisieren, wenn die Voraussetzung gilt. Beim unten in $\S 2.2 \mathrm{zu}$ behandelnden Pseudopartizip findet sich bei 2-rad. Verben ebenfalls ein Langvokal, dessen Qualität dagegen nicht mit den Vokalen der anderen Verbalklassen übereinstimmt. Weiter: Der Unterschied zwischen zwei Akzentuierungstypen, den die genannten Verbalklassen mit mehr als 2 Wurzel- bzw. Stamm-Konsonanten machen, bliebe jedoch auch damit unrealisiert, ${ }^{45}$ es sei denn, bislang von niemandem auch nur in Erwägung gezogen und auch nach den allgemeingültigen Regeln der Vokalisation nicht zu erwarten, statt des Langvokals seien zwei unmittelbar aufeinander folgende Kurzvokale anzunehmen, von denen fallweise der erste, fallweise der zweite betont wäre. Näher liegt somit der Schluss, dass Verben mit weniger als 3 Wurzel- und Stammkonsonanten geringere Formenbildungsmöglichkeiten haben, also fallweise Unterschiede nicht zum Ausdruck bringen, die bei Verben mit mehr als 2 Wurzel- und Stammkonsonanten zum Ausdruck gebracht werden können. Im Einzelnen:

(4.1) Infinitive des Typs (1) = (2) sind sowohl für fientische als auch für Zustandsverben belegt. ${ }^{46}$

(4.2) Infinitive des Typs (3) =(4) sind sowohl für transitive als auch für intransitive fientische Verben belegt. $^{47}$

(5) Ob bei infirmen Verben das finale, nicht zur Wurzel gehörige $t$ anstelle eines dritten (bzw. vierten) Radikals steht, kann man bezweifeln. Es gibt nämlich Infinitive mit $i$-Vokalisation, die möglicherweise beides zugleich haben, den finalen schwachen Radikal und ein zusätzliches $t$, so zum mindesten bei Verben

43 Osing 1976a, 54-56 (bis vor $\S 3.7, \mathrm{~b}$ ).

44 Osing 1976a, 62-63.

45 Ausdrücklich, wenn auch anders, bei Osing 1976a, 49, für die Infinitive mit $i$-Vokalisation konstatiert.

46 Osing 1976a, 36.

47 Osing 1976a, 49. 


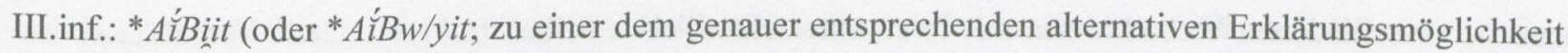
s. unten Punkt (6)). Von Interesse sind hier namentlich die Verben III.inf. (Solche Verben IV.inf., bei denen sich die Silbenzahl auf drei erhöht, sollen in unserem Zusammenhang wiederum außer Betracht bleiben, zumal gerade bei diesen die unten zu besprechende alternative Erklärung näher liegt.) Im Einzelnen:

(5.1) Infinitive dieses Typs sind (selten) bei transitiven, (häufig) bei intransitiven fientischen und (nicht häufig) bei Zustandsverben belegt. ${ }^{48}$

(6) Außer den endungslosen Infinitiven gibt es auch Infinitive mit der Endung $-w / y$ und der Endung $-w / y t$. Belegt sind:

(6.1) mit der Endung -w/y in der Nominalbildungsklasse I 8 śaçứm.uw/śaçútm.ut (Schenkel: śaçúum. พ̌ / śaçŭ́m.wut) ${ }^{49}$ Verben aus den unterschiedlichsten Verbalklassen (2-rad., II.red., 3-rad., III.inf., 4-rad.); ${ }^{50}$ (6.2) mit der Endung -w/y in der Nominalbildungsklasse I 9 śiçấm.aw/śiçấm.at (NB: für Nicht-Infinitive zu streichen, ${ }^{51}$ daher die Vokalisation über den Tonvokal hinaus bislang nicht rekonstruierbar) starke 3und 4-radikalige Verben; ${ }^{52}$

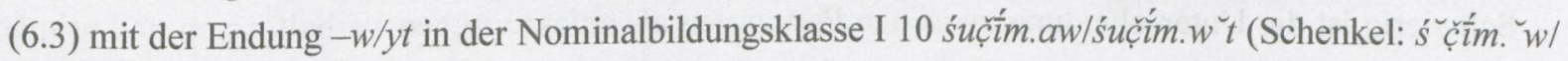

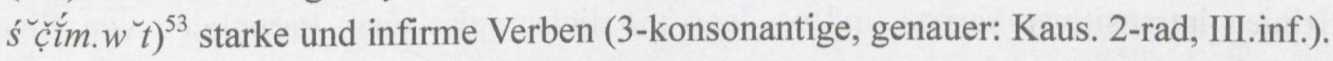

Hierher zu rechnen ist auch möglicherweise ein guter Teil der oben unter Punkt (5) angesprochenen Infinitive zu Verben III.inf., ${ }^{54}$ vor allem aber der entsprechenden Infinitive zu Verben IV.inf. ${ }^{55}$

\subsection{Pseudopartizip, en passant}

Es gibt mindestens eine Verbalform Pseudopartizip. Diese wird, wie man dies aus dem koptischen Nachfolger, dem Qualitativ, erschließen kann, in den verschiedenen Verbalklassen nicht einheitlich, nicht einmal analog gebildet. Auszugsweise ergibt sich für die 3. sg. m. das folgende Bild:

(1) starke dreiradikalige Verben und Verben III.inf.: *AắBC (w), z.B. *śắçm. “w „ist gehört“, *mắśni. ‘(w) ,ist geboren“,

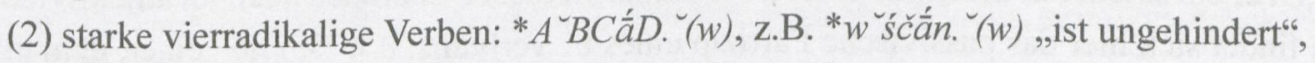

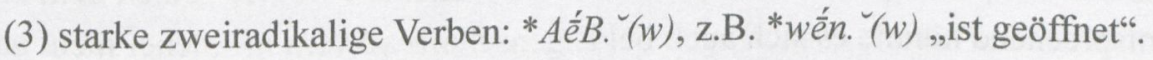

Ein Zusammenhang zwischen den verschiedenen Bildungsweisen ist schwer oder gar nicht erkennbar. Auch ist kein analoges Verhalten zwischen den Formen des Pseudopartizips und denen des Infinitivs erkennbar, selbst nicht bei den starken 2-radikaligen Verben, deren Langvokal anders als beim Infinitiv keine Besonderheit der Silbenbildung darstellt (der Tonvokal ist nach der Standardregel in offener Silbe lang, während beim Infinitiv die Länge des Tonvokals in geschlossener Silbe nicht der Standardregel folgt).

Grundsätzlich könnten sich Spuren der unterschiedlichen Silbenstrukturen in den hieroglyphischen Graphien finden lassen, zumal in der Schreibung und Nichtschreibung der Endung $-w$ bzw. $-y$. Strikt zu vermeiden ist bei der Suche nach der Realisierung der Endung eine vorausgreifende Verallgemeinerung

Osing 1976a, 52.

Zum Alternativansatz vgl. die Vokalisation der entsprechenden Substantive bei Schenkel 1983b, $206-207$.

50 Osing, 1976, 101-102.

51 Schenkel 1983b, 132.

52 Osing 1976a, 108.

53 Zum Alternativansatz vgl. die Vokalisation der entsprechenden Substantive bei Schenkel 1983b, 204-205.

54 Osing 1976a, 52-54.

55 Osing 1976a, 56-57. 
14 - THE BROWN WORKSHOP ON EARLIER EGYPTIAN GRAMMAR

über die verschiedenen Verbalklassen mit ihren unterschiedlichen Silbenstrukturen. Jede Verbalklasse ist zunächst einmal gesondert zu untersuchen.

\subsection{Partizipien, Adjektive}

Von jedem Verb können, belegt oder nicht belegt, mehrere Partizipien gebildet werden, aktivische und passivische (letztere, wie die Relativkonstruktionen zeigen, nicht nur von transitiven Verben), im älteren Ägyptisch mit und ohne Reduplikation bzw. mit und ohne Präfix. Reduplizierende sind über das Koptische generell nicht mehr rekonstruierbar, bleiben daher hier außer Betracht. Die Bildungsweise der einzelnen aktivischen bzw. passivischen Partizipien ist nicht einheitlich. Das lässt sich fallweise bereits an den Graphien ablesen, namentlich am Sonderfall des merkmallosen/präteritalen Partizips Passiv der 2-radikaligen Verben, das anders als das entsprechende Partizip anderer Verbalklassen Reduplikation zeigt (z.B. hmm(.i) "nicht gekannt" neben III.inf. mri.y ,geliebt" aus einer Verbalklasse, in der in anderen Fällen Reduplikation vorkommt).

Schwierig ist die Unterscheidung zwischen Partizipien als adjektivischen Verbalformen und primären Adjektiven. Partizipien intransitiver Verben und so dann möglicherweise auch Partizipien von Eigenschaftsverben und nicht unbedingt sinnentsprechende primäre Adjektive bilden das Prädikat des Adjektivalsatzes; z.B. (jeweils als Beschreibung einer Ausgangssituation) mit Verbum der Bewegung: $p r$ śbh $m$ $r^{\prime} n(. i)$ wr.w ... „Ein Schrei kommt aus dem Mund der Großen ...“; ${ }^{\text {‘6 }}$ mit anderem intr. Verbum, parallel

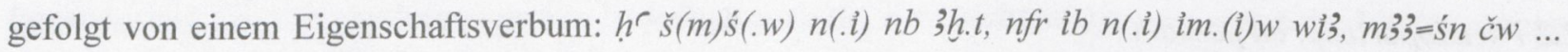
„Das Gefolge des Herrn des Horizontes jubelt, das Herz derer in der Barke ist guter Dinge, wenn sie dich sehen ... “. ${ }^{57}$ Aber keinesfalls jedes Adjektiv ist ein Partizip und nicht jedes Prädikat eines Adjektivalsatzes kann ein Partizip sein. Selbstverständlich sind (von Substantiven und Präpositionen) abgeleitete Nisben keine Partizipien, und sie sind dies dann auch nicht in ihrer Funktion als Prädikat im Adjektivalsatz. Das Paradebeispiel für die an sich nur sporadische prädikative Verwendung der Nisben ist das folgende: in mỉ.wï $s ́ w m n n \operatorname{irr}(. w)=f$, Ist er ein solcher in dem, was er tut? ${ }^{658}$ Fassbar ist dieser Gebrauch auch im adjektivierten Adjektivalsatz. Es findet sich hier das passivische Partizip eines tr. Verbums: ç.w `nh nb, „einer, dem alles Leben gegeben ist ${ }^{\text {“ }}{ }^{59}$ Dementsprechend könnte es sich auch bei Eigenschaftswörtern in solcher nicht ganz seltenen Verwendung um Partizipien handeln; z.B. bei $n f r-h r$, ,schöngesichtig“'. Eindeutig keine Partizipien liegen dagegen bei den adjektivierten Adjektivalsätzen mit Nisben als Prädikat vor, z.B. in šč.wï-hr „schildkrötengesichtig“. ${ }^{60}$ Es gibt aber auch eine ganze Menge primäre Adjektive, die keine Partizipien sein können, weil es entsprechende Verben nicht gibt (und auch in der älteren Sprachgeschichte nicht gab): $n b$ ,jeder", adjektivische Demonstrativa, adjektivische Numeralia, schließlich einzelne Lexeme wie rnp.w ,jung“" oder śmś.w ,ältest“. Anders als Partizipien werden diese grundsätzlich nur attributiv gebraucht und nicht prädikativ, auch nicht als Prädikat im Adjektivalsatz. Dann stellt sich die Frage, ob tatsächlich jedes Adjektiv, zu dem es ein inhaltlich passendes Verb gibt, als Partizip zu gelten hat oder ob nicht umgekehrt das Verb vom Adjektiv abgeleitet ist. Schließlich stellt sich, wie wir sehen werden, die Frage, ob es nicht von ein und derselben Wurzel beides geben kann, ein verbales Partizip und ein primäres Adjektiv.

58 CT IV, 288-89a; zur Interpretation der Textstelle s. Schenkel 2008a, 98-103.

59 Lacau und Chevrier 1956-69, Taf. 19, Szene 15 ( $\$ 210)$.

60 (Substantiviert) CT V, 325i B2L. 
Bei Osing 1976a stellt sich die Lage bei Adjektiven und Partizipien so dar (in Klammern die rekonstruierten Lautformen nach Schenkel 1983b):

(1) Aktiv

(1.1) Aktivische Partizipien bzw. Adjektive mit 3 Wurzel- bzw. Stamm-Konsonanten (3-rad., teilweise II.red. und III.inf.) sind in mindestens 6 Nominalbildungsklassen belegt:

Klasse II 1 śáḉcim/śắḉm.at „Adjektive und (perfektische) aktive Partizipien“ (śáç̌im/śắç̌m. ‘ t)

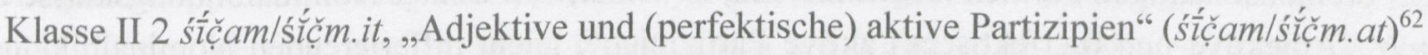

Klasse II 3 śúụçim/śŭç̌m.ut, „Adjektive“ (śúçcim/śŭ́çm. $t)^{63}$

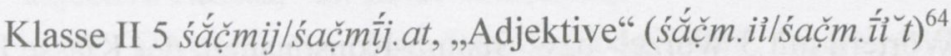

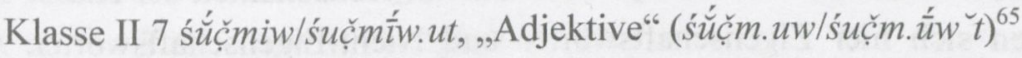

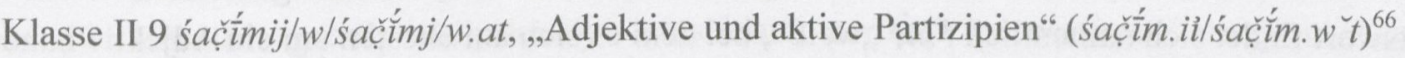

Die Belege aus Osings Klasse II 8 śaçấmuw/śaçắmw. ̌t, „Adjektive“, ${ }^{67}$ sind bei Schenkel 1983b, Klasse II 5 śắçm.ỉ/śaçm. ́̂̉ ̌ t zugeordnet. ${ }^{68}$ Hinzu kommt Klasse II 10, unter der Osing Adjektive/Partizipien nicht anführt (dazu s. unten (1.5)).

Dass Osing bei den drei Nominalbildungsklassen II 3, II 5 und II 7 von „Adjektiven“ spricht und nicht, wie bei den anderen Nominalbildungsklassen, von "Adjektiven und (perfektischen) aktiven Partizipien“ (o.ä.), hängt offensichtlich damit zusammen, dass die in den drei Klassen belegten Wörter alle

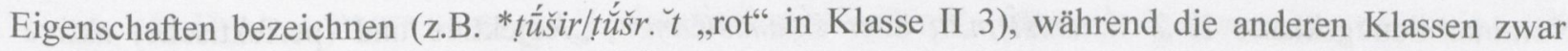

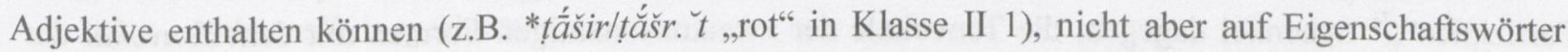
spezialisiert sind. In allen Fällen gibt es aber entsprechende (Eigenschafts-)Verben (z.B. zu *túúšir/țúšs. $\breve{t}$ „,rot" von Klasse II 3 und * țáširlțắšr. $t$,,rot" von Klasse II 1 das (Eigenschafts-)Verb ț̌rr „rot sein"). Warum

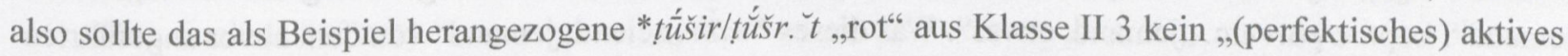
Partizip“ sein können? Andererseits könnte man sich natürlich auch fragen, ob das als Beispiel herangezogene Verb țšr „rot sein“ tatsächlich zwei Partizipien gleicher Bedeutung bildet, ob es also nicht neben dem vom Verbum abgeleiteten Partizip ein von der Wurzel abgeleitetes, primäres Adjektiv gibt. Auf diese Frage wird zurückzukommen sein.

(1.2) „(Perfektische) aktive Partizipien“ bzw. „Adjektive“ mit 2 Wurzel- bzw. Stamm-Konsonanten (2-rad., teilweise II.red.) haben naturgemäß (2-rad.) oder möglicherweise (II.red.) einen Vokal weniger als solche

61 Osing 1976a, 128-30 (3-rad., III.inf.; zu den II.red. vgl. die „Kurzformen“, s. unten (1.3)) (Schenkel 1983b, 154 und 156, evtl. auch 157 und 158).

62 Osing 1976a, 142-43 (II.red., III.inf., nicht 3-rad.; zu den „Kurzformen“ s. unten (1.3)) (Schenkel 1983b, 162 und 163-64, evtl. auch 165).

63 Osing 1976a, 149 (3-rad., problematisch II.red. und III.inf. mit drittem Konsonanten , j“) (Schenkel 1983b, 158 und 160, evtl. auch 161 (3-rad.)); zu den II.red. und III.inf. vgl. die „Kurzformen“ (s. unten (1.3)).

64 Osing 1976a, 161-62 (II.red., 3-rad., III.inf.) (Schenkel 1983b, 169-70).

65 Osing 1976a, 179-80 (II.red., 3-rad., III.inf.) (Schenkel 1983b, 171, evtl. auch 172 und 173; bei III.inf. teilweise stammbildendes $w$ ).

66 Osing 1976a, 196-97 (II.red., 3-rad., III.inf.) (Schenkel 1983b, 176, versehentlich unter "II.gem." stehend; bei III.inf., Schenkel 1983b, 175, möglicherweise stammbildendes $w$ ).

67 Osing 1976a, 188.

68 Es dürfte der Hinweis nicht ganz unnütz sein, dass es sich bei dem Götternamen hấruw „Horus“, der gerne und in der Sache auch durchaus richtig als „,der Ferne“ erklärt wird, nicht um ein substantiviertes Adjektiv handelt, sondern um ein primäres, direkt aus der Wurzel abgeleitetes Substantiv (so Osing 1976a, 185). 
mit 3 Wurzel- bzw. Stamm-Konsonanten. Belegt ist u.a. A Nominalbildungsklasse der 2-radikaligen Verben oder entspricht sie - semantisch? - einer bestimmten Nominalbildungsklasse der 3-radikaligen (etc.) Verben? Osing rechnet, den Tonvokal der zweikonsonantigen Formen mit dem Nachtonvokal der dreikonsonantigen Formen identifizierend, $A \check{\imath} B / A \bar{\imath} B . t$ zu seiner Nominalbildungsklasse Klasse II 1 śáç̌im/śăç̌m.at, älter śáạcim/śắç̌im.at, „Adjektive und (perfektische) aktive Partizipien“, ${ }^{69}$ Schenkel dagegen, die Tonvokale der zweikonsonantigen Formen mit dem Tonvokal der dreikonsonantigen Formen identifizierend, zu seiner, von Osings Nominalbildungsklasse II 2 śíç̌am/š́̌ç̌m.it, „Adjektive und (perfektische) aktive Partizipien“, leicht differierenden Nominalbildungs-

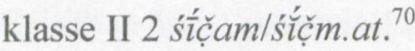

Wie bei den unter (1.1) behandelten Wörtern mit 3 Wurzel- bzw. Stamm-Konsonanten der Klasse II 1 (Osing) bzw. II 2 (Schenkel) finden sich hier Eigenschaftswörter und Nicht-Eigenschaftswörter. Als Eigenschaftswort findet sich namentlich *hĭčč „weiß“, bei dem es sich am ehesten um ein Adjektiv handelt, obgleich ein Partizip „weiß seiend“ nicht ganz auszuschließen ist. Ansonsten finden sich NichtEigenschaftswörter, so namentlich *mĭn ,bleibend“, *rĭh „,wissend“, *w wh ,seiend, existierend“, bei denen es sich um Partizipien handeln wird.

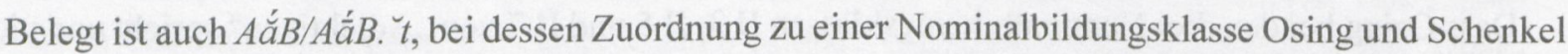

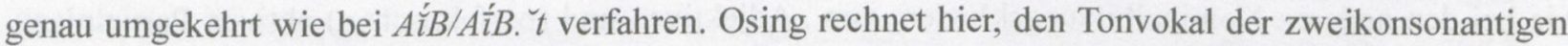
Formen mit dem Nachtonvokal der dreikonsonantigen Formen identifizierend, $A a ̆ ́ B / A \bar{a} B . \breve{t}$ zu seiner

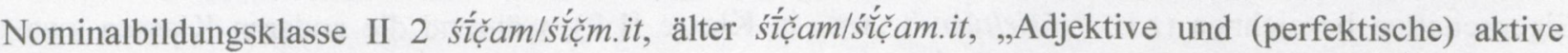
Partizipien“, ${ }^{71}$ Schenkel dagegen, die Tonvokale der zweikonsonantigen Formen mit dem Tonvokal der dreikonsonantigen Formen identifizierend, zu seiner Nominalbildungsklasse Klasse II 1 śáçčim/śắçm. ‘t (nach Osing „Adjektive und (perfektische) aktive Partizipien“). ${ }^{72}$ Der einzige Beleg ist *wắn „,seiend; jemand“, kopt. oyon etc. ,jemand, etwas“. Was dieses *wắn „seiend; jemand“ von dem zuvor angeführten *wĭn „seiend, existierend“ unterscheidet, ist nicht sicher zu entscheiden. Könnte es sich bei einem der beiden um ein Partizip, bei dem anderen um ein (substantiviertes) Adjektiv handeln? ( $\mathrm{Zu}$ den Bedeutungsfeldern des Adjektivs s. unten (1.4).)

(1.3) Instruktiv sind in diesem Zusammenhang die Wörter zu Wurzeln III.inf., bei denen man neben den Formen mit 3 Konsonanten Kurzformen ohne den schwachen Radikal findet, namentlich Bildungen des

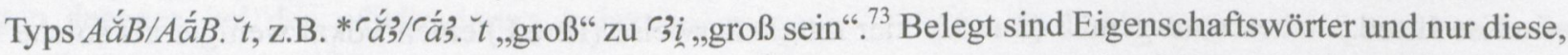
so dass man den Eindruck gewinnt, dass es sich um eine eigene Nominalbildungsklasse „Adjektive“ handelt, die weder mit Osings Nominalbildungsklasse II 2 śççam/śl̆çcm.it, „Adjektive und (perfektische) aktive Partizipien“, noch mit Schenkels Nominalbildungsklasse II 1 śáç̌im/śăç̌m.at (nach Osing „Adjektive und (perfektische) aktive Partizipien") zu gleichen ist, da beide Nominalbildungsklassen nicht auf Eigenschaftswörter spezialisiert sind. Dem sei ergänzend hinzugefügt, dass es auch unter den Nominalbildungen mit einer konsonantischen Endung eine Klasse gibt, die ausschließlich „Adjektive“ enthält:

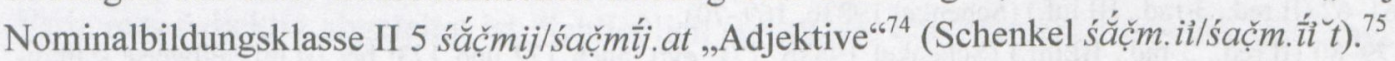


(1.4) Belegt sind nach Osing in einem Teil der Verbalklassen „Adjektive und (perfektische) aktive Partizipien“ bzw. „Adjektive und aktive Partizipien“, in einem anderen Teil der Verbalklassen dagegen nur „Adjektive“. Offensichtlich ergibt sich die Unterscheidung daraus, dass in letzterem Fall nur Eigenschaftswörter belegt sind, in ersterem dagegen Eigenschaftswörter nur unter anderem. Statt „Adjektive“ könnte man auch „Eigenschaftswörter“ sagen. Zu erwarten wären unter diesen Adjektiven/Eigenschaftswörtern nach den allgemein-linguistischen Beobachtungen bei Dixon 2004, aus denen Carsten Peust bereits die wichtigste Gruppe, (a), zitiert, ${ }^{76}$ vor allem Wörter aus den folgenden Bedeutungsfeldern:

(a) „There are four core semantic types, which are typically associated with both large and small adjective classes, " im Einzelnen (Zitat):

1. Dimension - 'big', 'small', 'long', 'tall', 'short', 'wide', 'deep', etc.

2. AGE - 'new', 'young', 'old', etc.

3. VALUE - 'good', 'bad', 'lovely', 'atrocious', 'perfect', 'proper(/real)' etc. (And also words such as 'odd', 'strange', 'curious', 'necessary', 'crucial', 'important', 'lucky')

4. COLOUR - 'black', 'white', 'red', etc. ${ }^{77}$

(b) „A number of peripheral semantic types are typically associated with medium sized and large adjective classes, “, im Einzelnen (Zitat):

5. PHYSICAL PROPERTY - 'hard', 'soft', 'heavy', 'wet', 'rough', 'strong', 'clean', 'hot', 'sour', etc. And a sub-class referring to corporeal properties, e.g. 'well', 'sick', 'tired', 'dead', 'absent'

6. HUMAN PROPENSITY - 'jealous', 'happy', 'kind', 'clever', 'generous', 'cruel', 'proud', 'ashamed', 'eager', etc.

7. SPEED - 'fast', 'quick', 'slow', etc. ${ }^{78}$

(c) „A number of other semantic types are associated with large adjective classes in some languages. These include:" (Zitat)

8. DifFICULTY - 'easy', 'difficult', 'tough', 'hard', 'simple', etc.

9. SIMILARITY - 'like', 'unlike', 'similar', 'different(/strange)', 'other', etc.

10. QUALIFICATIONAL - 'definite', 'true', 'probable', 'possible', 'likely', 'usual', 'normal', 'common', 'correct', 'appropriate', 'sensible', etc.

11. QUANTIFICATION - 'all(/whole)', 'many', 'some', 'few', 'only', 'enough', etc.

12. POSITION - 'high', 'low', 'near', 'far/distant', 'right', 'left(/strange)', 'northern', etc.

13. CARDINAL NUMBERS - (In some languages these constitute a separate word class.) And 'first', 'last' (together with other ordinal numbers). ${ }^{679}$

In Osings Nur-Adjektiv-Klassen (II 3, II 5, II 7) stellt sich der Befund nach Osing bzw. Schenkel so dar (Vokalisation der Einzelbelege, wenn möglich, nach Schenkel; Übersetzungen in Anlehnung an Osing; bei aus der substantivierten Form erschließbaren Adjektiven ist die Übersetzung mit „*" markiert):

Klasse II 3 śúç̌im/śúḉm.ut „Adjektive“ (Osing 1976a, 148-50) bzw. śúúçim/śŭç̌m. ‘ (Schenkel 1983b, 158 und 160, evtl. auch 161 (3-rad.)); zu den „Kurzformen“ der II.red. und III.inf. aus oben (1.3) s. unten (Klasse II 1 oder Klasse II 2, Kurzformen):

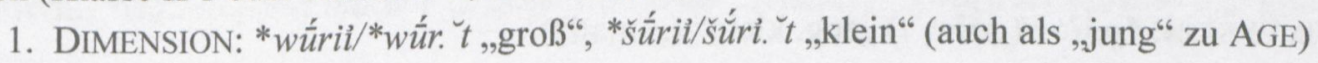

2. AGE: s. 1. DIMENSION 


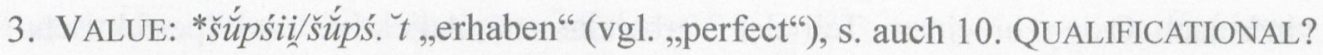

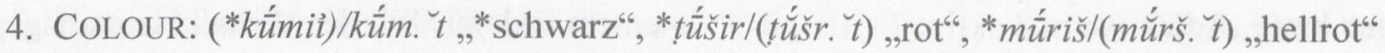

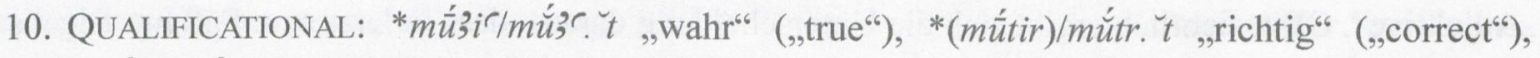

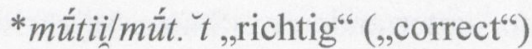

Klasse II 5 [+ II 8] śắçmij/śaçmî́j.at [śaç̌ámuw/śaçắmw. ̌t] „Adjektive“"(Osing 1976a, 161-63 und 188-

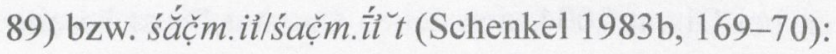

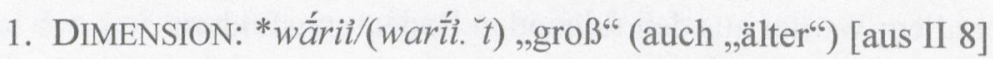

2. AGE: s. 1. DIMENSION

3. VAlue: s. 5. Physical Property, 6. Human Propensitiy, 10. QualificATIONAL?

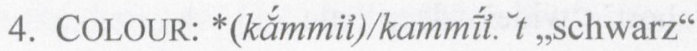

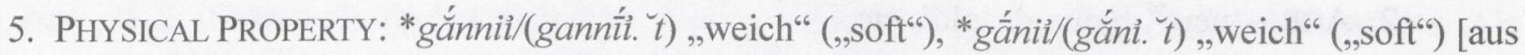

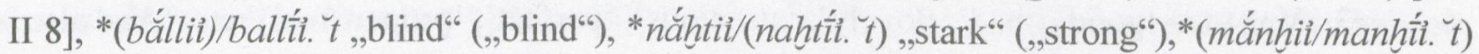

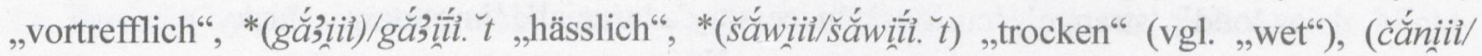

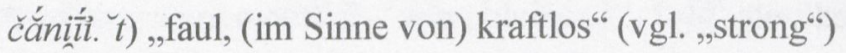

6. HUMAN PROPENSITY: *sắwgiil/sawgíli. $t$, ,töricht"“

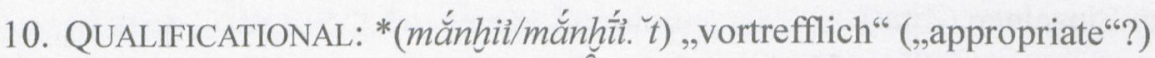

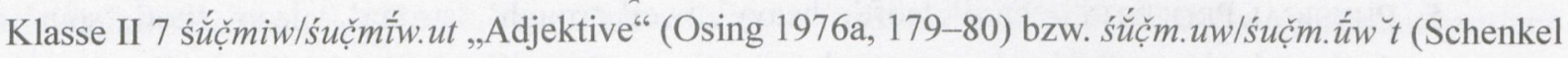
1983b, 171, evtl. auch 172 und 173), bei III.inf. teilweise stammbildendes $w$ :

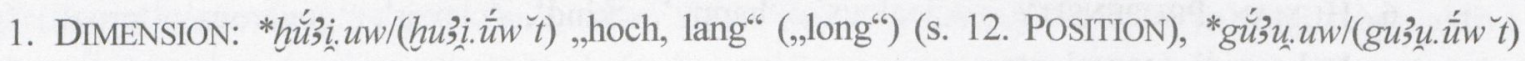
„eng, schmal" (vgl. „wide“)

2. AgE: zu einem denkbaren Beleg s. 5. PHySICAL PROPERTY

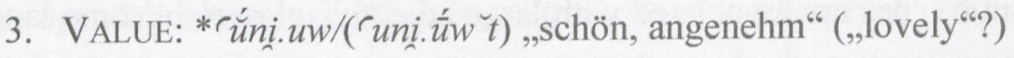

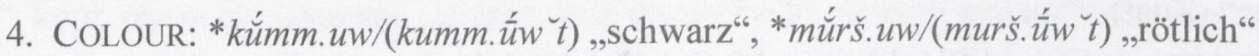

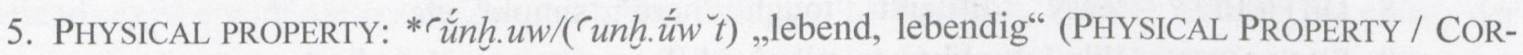
PORAL PROPERTY, vgl. „dead“; oder als Gegensatz zu „verstorben“ zu AGE?)

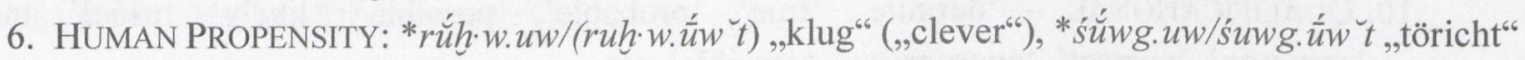
(vgl. „clever")

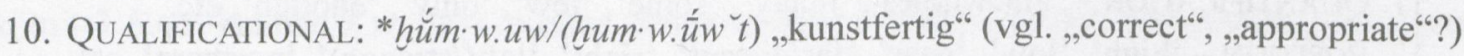

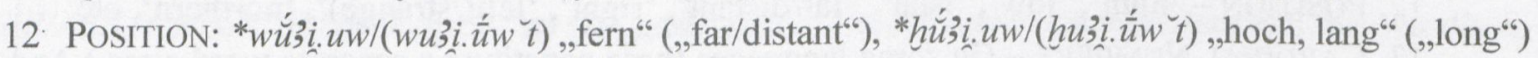
(s. 1. DIMENSION)

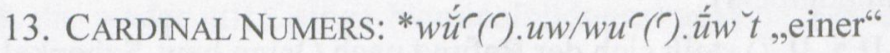

Bei Osings Auch-Adjektiv-Klassen (II 1, II 2, II 9) stellt sich der Befund nach Osing bzw. Schenkel so dar (Vokalisation der Einzelbelege, wenn möglich, nach Schenkel; Übersetzungen in Anlehnung an Osing; bei aus der substantivierten Form erschließbaren Adjektiven ist die Übersetzung mit "* “ markiert): Klasse II 1 śáç̌im/śăç̌m.at, „Adjektive und (perfektische) aktive Partizipien“ (Osing 1976a, 128-30) (śáạcim/śắçm. ‘t, Schenkel 1983b, 154, 156). Als Adjektive kommen möglicherweise in Frage:

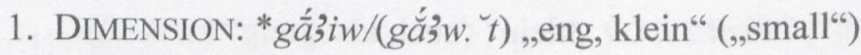

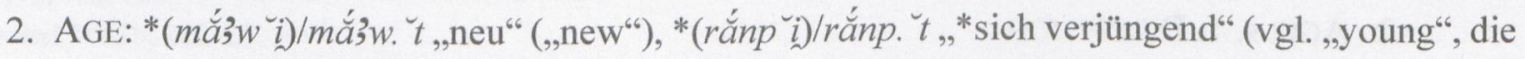
deutsche Übersetzung suggeriert, möglicherweise zu Recht, ein Partizip)

3. VALUE: Hierher gehört möglicherweise die Mehrzahl der zahlreichen Belege; als z.B. *bấỉin/ bắin. t „,schlecht, schlimm“ (,bad"), *náfir /nắfr. t „,schön, gut" (,good“, „lovely“)

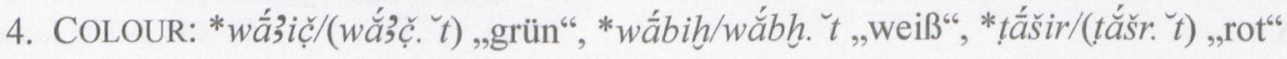




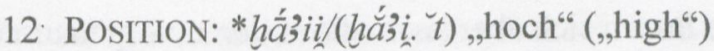

Nicht Adjektive, sondern Partizipien liegen namentlich vor bei Wörtern transitiv-aktivischer Art:

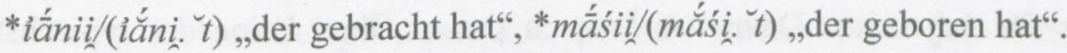

Dass Adjektive und Partizipien lautsprachlich nicht voneinander unterschieden sind, kann zwei unterschiedliche Ursachen haben. Zum einen kann es sich bei einem Teil der Adjektive genetisch um Partizipien handeln. Das könnte namentlich der Fall sein bei den Farbwörtern wấ,iç̌/wắ’ç. $t$,grün“ und wấbihh/wắbh. ‘ „ „weiß“. Bei ersterem könnte die ursprüngliche Bedeutung ,grünend, gedeihend“ sein, das Wort also etymologisch ein Partizip des Verbs w'ç̌ „gedeihen, grünen“. Bei letzterem liegt eine entsprechende Erklärung auf der Hand: Zum Farbwort wird wábih̆wắbh. ̌t erst im Verlauf der späteren ägyptisch-koptischen Sprachgeschichte, in der es an die Stelle von hé̌́č des älteren Farbworts für „weiß“, tritt. Eine alternative Erklärung, die in jedem Fall zu erwägen ist, ist die, dass im Ägyptischen infolge der Durchführung des Zweisilbengesetzes die Adjektive, die ursprünglich (im maskulinen Singular) einen kurzen Tonvokal hatten, mit den Partizipien, die von vornherein einen langen Tonvokal hatten, zusammenfielen. ${ }^{80}$

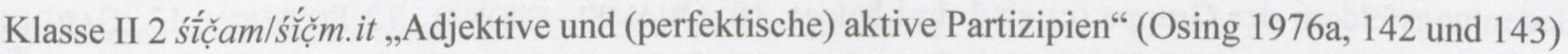
bzw. śíç̌am/śĭ́čm.at (Schenkel 1983b, 162-64). Als Adjektive kommen möglicherweise in Frage:

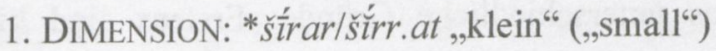

3. VALUE: * 'rinai/( ( ' ini.at) „schön, angenehm“(,lovely“?)

Nicht Adjektive, sondern Partizipien liegen namentlich vor bei Wörtern der Bewegung: *if́xai/

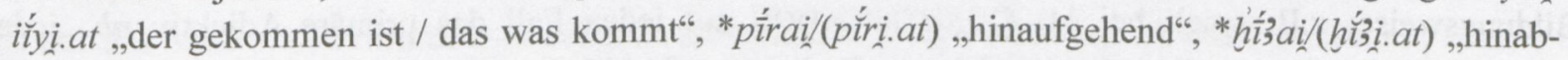
steigend".

Infrage kommt auch als Partizip eines transitiven Verbs (bei Osing und Schenkel als Substantiv eingeordnet): *(míśai $) / m u ́ s$ íl.at ,(der/)die geboren hat". ${ }^{81}$

In Anbetracht der schwachen Beleglage bei den „Adjektiven“ stellt sich die Frage, ob es sich bei diesen nicht um Partizipien der dazu passend vorhandenen Eigenschaftsverben handelt. Wäre dies so und wäre, wie erörtert, die Nominalbildungsklasse II 1 auf ursprünglich zwei Nominalbildungsklassen zu verteilen, wären Adjektive und Partizipien ursprünglich unterschiedlich gebildet worden.

Klasse II 9 śaç̌̆ímij/w / śaç̌̆́mj/w.at „Adjektive und aktive Partizipien“ (Osing 1976a, 196-97) bzw.

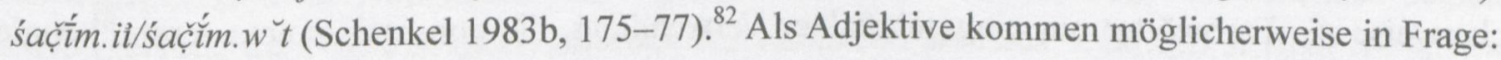

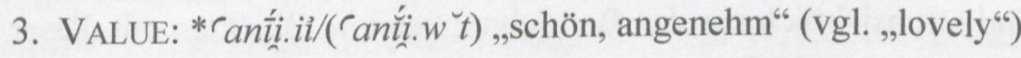

5. PHYSICAl PROPERTy / CORPORAL PROPERTY: (4-rad.) * gabgíb.iỉ/(gabgíb.w̌t) „in die Knie gesunken, lahm“" (vgl. z.B., ,sick“)

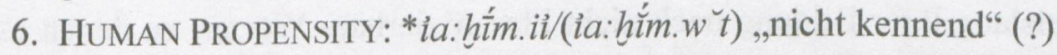

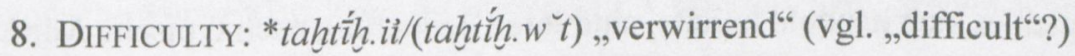

10. QUALIFICATIONAL: *(ia:mín.iỉ)/ia:mĭn.w ${ }^{ॅ}$, „*dauernd“" (vgl. „normal“, „,common“?)

Nicht Adjektive, sondern Partizipien liegen namentlich vor bei intr. Vorgänge bezeichnenden

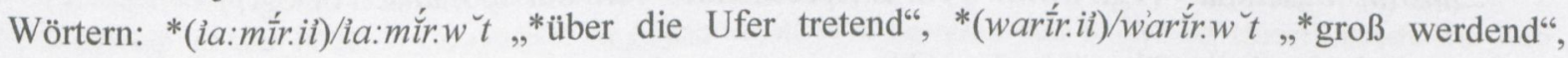

80 S. Knauf 1986; s. auch Satzinger 2012, 471.

81 Osing 1976a, 138; Schenkel 1983b, 164.

82 Randbemerkung: Möglicherweise kann man die Belege der Klasse II 9 auf "Adjektive” beschränken. Die Nicht-,,Adjektive“ lassen sich nämlich alternativ als Abstrakta der Klasse I 10 zuweisen, wenn man diese Klasse, die Osing als śuç̌́maỉ/w /

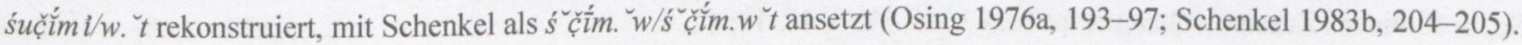




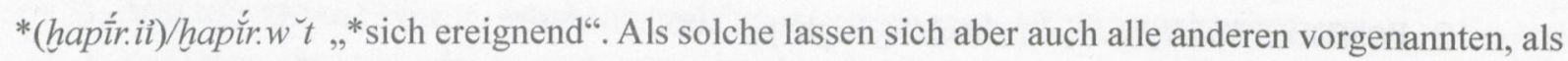
Adjektive in Erwägung gezogenen Wörter verstehen, namentlich solche mit $i$-Präfix, das für Partizipien bestens belegt ist, nicht aber für Adjektive. Dazu würde auch passen, dass keine der typischsten Adjektive der Gruppe (a) belegt sind, ausgenommen allenfalls 3. VALUE.

Klasse II 1 oder Klasse II 2, Kurzformen des Typs $A \stackrel{a}{B} / A \stackrel{a}{a} B . \breve{t}$ (s. oben (1.3)): ${ }^{83}$

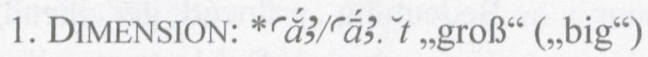

$$
\begin{aligned}
& \text { 3. VALUE: *çắw/çấw. „t „schlecht“ („,bad“) }
\end{aligned}
$$

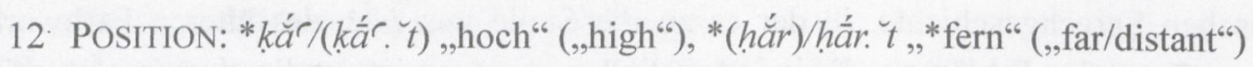

In den angeführten Nominalbildungsklassen finden sich Belege für alle vier Bedeutungsfelder der Gruppe (a), 1. Dimension, 2. AgE, 3. VAluE, 4. COlOUR. Von den drei Bedeutungsfeldern der Gruppe (b) sind zwei belegt, 5. PHySICAL Property, 6. Human Propensity, nicht jedoch 7. SPEED. Von den sechs Bedeutungsfeldern der Gruppe (c) sind drei belegt, 10. QUALIFICATIONAL, 12 POSITION, 13. CARDINAL NuMBERS, drei dagegen nicht belegt, 8. DifFICULTY, 9. SimILARITY, 11. QUANTIFICATION. Dass ein Teil der Bedeutungsfelder nicht besetzt ist, hat fraglos unterschiedliche Gründe: Erstens sind hier nur vokalisierbare Wörter herangezogen worden, die unter den einschlägigen Wörtern im Allgemeinen eine Minderzahl darstellen. Zweitens fallen in die genannten Bedeutungsfelder auch Wörter anderer Bildungsweise; z.B. spielt bei 11. QUANTIFICATION auf jeden Fall das primäre Adjektiv $n b$,jeder" (,all/whole“) die Hauptrolle, oder es spielen im Bedeutungsfeld 12. POSITION auch Nisben eine wesentliche Rolle, z.B. wnm.i „rechts“ (,right“), i’’b.i „links/östlich“ („left“), mh.ti „nördlich“ („,northern“), oder bei 9. SIMILARITY vor allem das Substantiv (!) ky.i „Anderer“ („other"). Nicht auszuschließen ist schließlich, dass der Verfasser einem Bedeutungsfeld nicht die richtigen ägyptischen Wörter zugeordnet hat.

Als primäre Adjektive einzuschätzen über die eingangs genannten hinaus ( $n b$,jeder", unter 11. QUANTIFICATION, ein Teil der Kardinal- und Ordinalzahlwörter, unter 13. CARDINAL NUMBERS, sowie ein Teil der Demonstrativa) sind solche Eigenschaftswörter, zu denen es keine entsprechenden Verben gibt oder entsprechende Verben aus den Eigenschaftswörtern, „zurückgebildet“ sein könnten. Unter anderem sind dies möglicherweise die Farbwörter nur eingeschränkter Verwendung, die non-basic colour terms. ${ }^{84}$ Andere dieser Gruppe von Farbwörtern sind offensichtlich aus den Bezeichnungen von Objekten mit dieser Farbe, also aus Substantiven abgeleitet. Für solche Ableitungen muss man nicht mit dem Umweg über Verben rechnen, da die Ableitung von Verben aus Substantiven ebenfalls kein Standardverfahren ist. Diese Farbwörter, aber auch andere bislang ihrer Bildung nach nicht klassifizierbare Wörter, zu denen es keine Verben gibt, um deren Partizipien es sich handeln könnte, sind ihrer Bedeutung nach typische Adjektive:

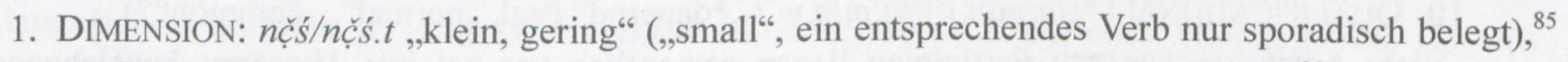
hnśs/hnś.t „schmal“ (vgl. „wide“, ein entsprechendes Verb nur sporadisch belegt?) ${ }^{86}$

2. AGE: śmś.w/śmś.t, ,ältest“ (vgl. „old“)

83 Osing 1976a, 143; Schenkel 1983b, 155 und 157.

84 S. etwa Schenkel im Druck.

85 Z.B. eindeutig im Pseudopartizip: $\operatorname{ir}(. t i)$ nç̌s.w ,die Augen sind schwach“ (Ptahhotep 11).

86 So, in ähnlichen Zusammenhängen, pEbers 36,$6 ; 37,6 ; 41,14 ; 42,1$. 
4. COLOUR (non-basic colour terms, fast alle im Rot(-Gelb)-Bereich, dem Bereich der warmen Farben, zitiert hier das Maskulinum als Lemma): ${ }^{87} \check{c} m s ́$,rot“, genauer etwa „knall-rot“" (auch entsprechendes Verbum belegt ${ }^{88}$ ), inś „rot, (genauer etwa) rötlich“" (ein entsprechendes Verb mit kausativer Bedeutung erst griechisch belegt, ${ }^{89} \check{c}(w) r$, wtr ,rot“; neben Verben, aber abgeleitet von StoffBezeichnungen: $\check{c}\ulcorner b$,holzkohlen-artig, holzkohlen-farben, raben-schwarz" (zu č $c b . t$, ,Holzkohle“),

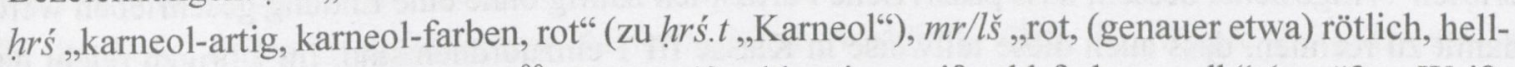

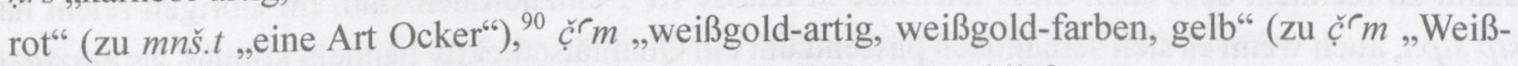
gold“), bei letzterem die Lesung als Nisba çॅ $m(. i)$ nicht auszuschließen.

Adjektive ohne den Endungskonsonanten $w$ können in eine der drei Nominalbildungsklassen gehören, für die Adjektive bezeugt sind (zur Beleglage s. oben (1.1)):

Klasse II 1 śáḉčim/śắḉm.at „Adjektive und (perfektische) aktive Partizipien“ (śáçcim/śăç̌m. ‘t)

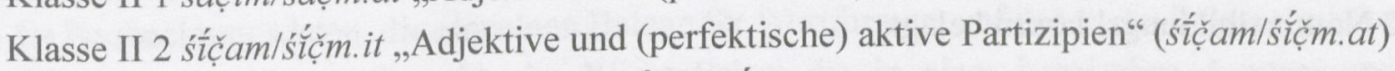

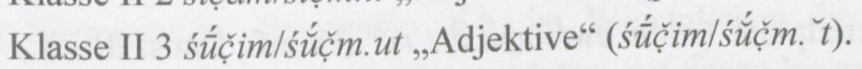

Darüber hinaus kommt als eine weitere Nominalbildungsklasse, für die Adjektive bislang nicht belegt sind,

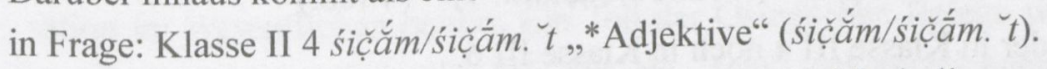

Adjektive mit dem Endungskonsonanten $w$ im Maskulinum und ohne Endungskonsonanten $w$ im Femininum, wie belegt in śmś.w/śmś.t, „ältest", passen nur zu einer Nominalbildungsklasse, für die Adjektive bislang nicht belegt sind: Klasse II 10 śaçŭ́m/śaçứm. $t$ „Adjektive“ (śaçứmew/śaç̌úm. $t$ < *śaçúm.w $w$ t). ${ }^{91}$

Ist nur das Maskulinum mit dem Endungskonsonanten $w$ belegt, nicht jedoch das Femininum, könnte letzteres statt der Endung $t$ auch die Endung $w t$ haben. In diesem Fall kommt zusätzlich die Nominal-

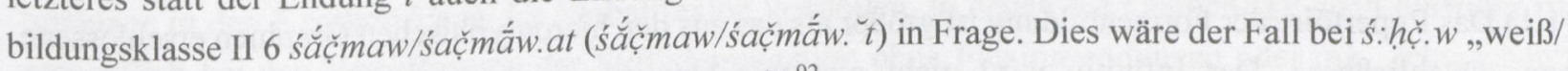
hell werden lassend" und $s: w, c ̧ c . w$ „ergrünen lassend“ .92

(1.6) Es sei die Vermutung gewagt, Adjektive wie śmś.w/śmś.t „ältest“", die keiner adjektivischen Nominalbildungsklasse zugewiesen werden können, und/oder Adjektive, zu denen es kein entsprechendes Verb gibt, können nicht als Prädikat im Adjektivalsatz stehen. Es läge damit die Verallgemeinerung auf der Hand, primäre Adjektive würden nur attributiv verwendet, in prädikativer Verwendung im Adjektivalsatz stünden dagegen generell Partizipien, also adjektivische Verbalformen, oder Nisben, also sekundäre Adjektive (geläufig die Nisba n.i zum Ausdruck des Besitzverhältnisses, z.B. $n(. i)$ śi (i) $m(. i)-r$ '-pr(.w) < $*_{n}(. i)$ (i) $m(. i)-r$ '-pr(.w) śï ,sie (die Gegend) gehört dem Vermögensverwalter“ (wörtl.: ,sie ist zugehörigzum-Vermögensverwalter" $){ }^{93}$

(2) Passiv

(2.1) Passivische Partizipien bzw. Adjektive mit 3 Wurzel- bzw. Stamm-Konsonanten (3-rad., teilweise III.inf.) sind in mindestens 2 Nominalbildungsklassen belegt:

S. etwa Schenkel im Druck, mit weiterführenden Literaturverweisen.

Pseudopartizip: pSmith 3, 20; 3, 10; Prädikat im Adjektivalsatz (von Interesse, falls es sich bei einem solchen, wie oben erwogen, stets um ein Partizip handelt): pSmith 13, 20; 14, 12.

Wb. I, 100,5.

Erst koptisch ein basic colour term.

Osing 1976a, 201-203 (Schenkel 1983b, 177-78, zum Ansatz des Femininums 96).

Cairo CG 20538, II c 12-13; hierzu auch Uljas 2007b, 235 mit Fn. 27.

Bauer B 1, 16/alt=47/neu. 


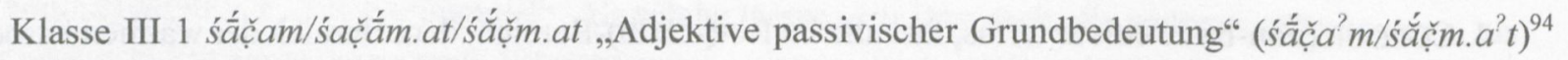
(nur 3-rad.)

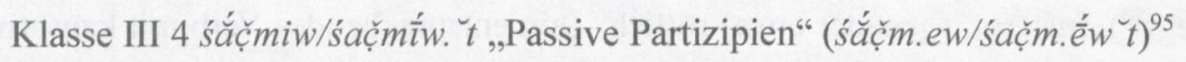
Der Ansatz der Klasse III 1 als eine Adjektiv-Bildung basiert auf einem einzigen Beleg, śtp/stp.t ,auserlesen". Angesichts dessen, dass passivische Partizipien häufig ohne eine Endung geschrieben werden, ist damit zu rechnen, dass auch diese teilweise in Klasse III 1 einzuordnen sind. Im Übrigen reicht natürlich $s t p / s t p . t$, auch wenn es sich semantisch passend um ein Qualitätsadjektiv handeln könnte, als einziger Beleg nicht dazu aus, Klasse III 1 als eine Adjektiv-Bildung abzusichern, da es sich bei dem Beleg genauso gut um ein passivisches Partizip handeln kann.

(2.2) Passivische Partizipien mit 4 Wurzel- bzw. Stamm-Konsonanten (IV.inf., 4-rad., Kaus. III.inf.) sind in mindestens 2 Nominalbildungsklassen belegt:

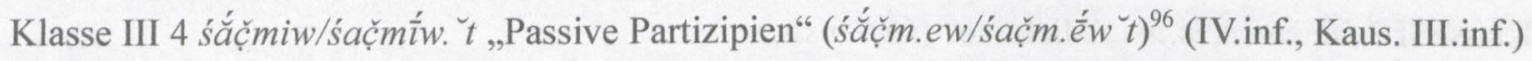

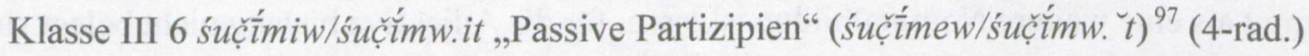

Adjektive sind nicht nachgewiesen, weder in Klasse III 4 noch in Klasse III 6.

\subsection{Zwischenbilanz}

Welche Einsichten kann man auf dem Umweg über die Vokalisation für die Interpretation der hieroglyphischen Graphien der Verbalformen gewinnen?

(1) Formen-Repertoire

(1.1) Das Repertoire der Formenbildung ist größer als das aus den hieroglyphischen Graphien erschlossene bzw. erschließbare. Der Infinitiv wird nicht nur, wie bekannt, in den verschiedenen Verbalklassen unterschiedlich gebildet - z.B. III.inf. in der Regel mit finalem $t$, 3-rad. ohne besonderes Merkmal —, es spielen vielmehr auch semantische Faktoren eine Rolle - z.B. $a$ - $a$-Vokalmuster eher bei transitiven Verben, $i-i$-Vokalmuster eher bei intransitiven Verben.

(1.2) Die Formenbildung der Verbalklassen ist teilweise eine je eigene, abhängig von den für Vokale verfügbaren Positionen zwischen den Konsonanten, teilweise wird mit Hilfe zusätzlicher Konsonanten z.B. $t$ beim Infinitiv der III.inf. - oder eliminierter schwacher Radikale - z.B. beim Infinitiv der IV.inf. ohne den schwachen Radikal - die Wortform auf die Länge der starken 3-rad. Verben, der umfangreichsten Verbalklasse, standardisiert.

(1.3) Die Formenbildung hängt innerhalb einer Wurzelklasse teilweise von semantischen Faktoren ab. Z.B. fehlt den Eigenschaftswörtern der III.inf., seien diese Partizipien oder primäre Adjektive, der schwache Radikal (Typ $A B$ ), während er bei Nominalbildungen sonst in der Regel vorhanden ist (Typ $A B i$ ).

(1.4) Selbst vokalisierbare Formen lassen sich nicht in jedem Fall eindeutig einer paradigmatischen Stelle zuordnen. Z.B. ist nicht definitiv entscheidbar, ob es sich bei nominalen Eigenschaftswörtern um adjektivische Verbalformen (Partizipien) oder primäre Adjektive handelt. 


\section{(2) Grenzen der Erschließbarkeit}

(2.1) Es kann weder auf der Basis der hieroglyphischen Graphien noch mit Hilfe einer Rekonstruktion der Vokale noch auf der Basis der Kombination beider Verfahren die Formenbildung der Verben vollständig erschlossen werden. Ganz im Gegenteil: Für die Masse der Verben ist die Beleglage in beiden Bereichen zu schwach, als dass man die Realität damit fassen könnte. Die Überlieferungslage ist brüchig. Z.B. sind in dem für die Vokalisation herangezogenen Material keine Auskünfte über die Vokale reduplizierender Partizipialformen zu gewinnen. Es gilt also, sich bei Verallgemeinerungen zurückzuhalten.

(2.2) Die Verfahren zur Rekonstruktion der Vokalisation bedürfen dringend der Konsolidierung. Man sollte sich nicht auf Dauer damit zufrieden geben, dass die Rekonstruktionen Osings und Schenkels voneinander differieren. Schließlich sind - was in unserem Zusammenhang nicht Gegenstand der Diskussion sein kann - die Beobachtungsdaten, die einzelnen Belege für Wortformen, auf denen die Rekonstruktionen basieren, im Einzelnen zu überprüfen, d.h. im Wesentlichen die in einer heroischen Anstrengung von Osing zusammengetragenen Beobachtungsdaten, auf denen bis zum heutigen Tag die Rekonstruktion der Vokalisation im Wesentlichen beruht. Ein Teil der Osingschen Belege wird einer genaueren Überprüfung nicht standhalten, namentlich ein Teil der als "spät" gekennzeichneten, unter denen die Belege aus dem schwierigen Papyrus BM 10808 eine nicht geringe Rolle spielen, aber auch ein Teil der Belege, die erst „kopt.“ fassbar sind.

\section{(3) Verallgemeinerung}

Problematischer noch als bei den Infinitiven, dem Pseudopartizip und den Partizipien muss man sich die Sachlage in den Kernbereichen der Verbalflexion vorstellen, in denen die Quellenlage noch dürftiger ist, namentlich bei den Verbalformen der Suffixkonjugation ohne Flexionsendungen oder mit „schwachen“, nicht konsequent geschriebenen Flexionsendungen. Nicht nur mit unterschiedlicher Formenbildung in den verschiedenen Verbalklassen muss man rechnen, sondern auch mit unterschiedlicher Formenbildung bei den einzelnen Verben. Abgesehen von sporadischen Informationen zur Vokalisation verfügt man über drei wesentliche Ansatzpunkte: die hieroglyphischen Graphien, den syntaktischen Zusammenhang und allgemein-linguistische Fragestellungen. Man sollte sich mit keinem dieser Ansatzpunkte in Sicherheit wiegen. Die hieroglyphischen Graphien sind, wenn nicht im Einzelfall fehlerhaft, ausreichend für die lautsprachlich korrekte Realisierung durch den des Ägyptischen bereits mächtigen Leser. Dieser erkennt intuitiv die Silbenstruktur und ergänzt intuitiv die nicht-geschriebenen Vokale, so wie z.B. jemand, der die arabische Schriftsprache beherrscht, in einen unvokalisierten arabischen Text im Allgemeinen an der richtigen Stelle die richtigen Imperfekt-Vokale einsetzen kann, die so wenig regelhaft sind, dass sie in den Wörterbücher explizit verbucht werden müssen. ${ }^{98}$ Die Nutzung des syntaktischen Zusammenhangs setzt ein Textverständnis voraus, über das der Ägyptologe oft nicht verfügt, ganz abgesehen davon, dass das Verständnis der Texte durch Korruptelen behindert sein kann. Beispielsweise stören, wie oben bereits gesagt, gedankenlose Ersetzungen von Pronomina durch Titel und Eigennamen den syntaktischen Zusammenhang. Linguistik-Import liefert Fragestellungen, deren Berechtigung am Quellenmaterial überprüft werden muss. Man darf sich nicht über die Mangelsituation bei den Textquellen hinwegsetzen. 


\section{Die Bestimmung Der WORTFORM AUS GRAPHiE Und KoTEXT: „DAS śç̌m=f" DES ÄLTEREN ÄGYPTISCH}

Im Allgemeinen bereitet es dem Ägyptologen keine allzu großen Schwierigkeiten, eine Wortform als z.B. Infinitiv, Pseudopartizip oder Partizip zu bestimmen - einmal abgesehen von dem zuvor besprochenen Problem der Unterscheidung von Partizip und Adjektiv im Prädikat des Adjektivalsatzes. Auch wenn die Wortformen fallweise an Flexionsendungen (oder dem Fehlen solcher, evtl. auch an Präfix, Reduplikation) zu erkennen sind, liefern doch bei einem guten Teil der Wortformen der syntaktische Zusammenhang und auch der Gedankengang eines Textes die besten Anhaltspunkte. Das gilt unter anderem auch für einen Teil der Verbalformen der Suffixkonjugation, selbst für das schillernde śḉm=f, „das śç̌m=f“, unter dem diejenigen Verbalformen der Suffixkonjugation pauschal oder doch zum Teil zusammengefasst werden, die allenfalls Flexionselemente aus „schwachen“ Konsonanten aufweisen. Es ist hier nicht der Ort, die heftige Diskussion über die Anzahl der voneinander zu unterscheidenden ,śç $m=f^{\prime \prime}$-Formen zu referieren oder gar fortzuführen. ${ }^{99}$ Der Fall ist hoffnungslos, wenn man eine Klärung auf der Basis allein vokalisierter Formen sucht. Nach den Erfahrungen, die man, wie oben dargestellt, anhand von Infinitiv, Pseudopartizip und Partizip/Adjektiv gewinnen kann, ist mit einer überreichlichen Formenbildung zu rechnen, die in keinem rechten Verhältnis zu dem schwachen in Graphien greifbaren Befund steht.

Nun ist zwar denkbar, dass die finiten Verbalformen stärker paradigmatisch durchgeordnet sind als die Nominalbildung, von der oben in $\S 2$ hauptsächlich die Rede war. Man könnte hier mit einem root-andpattern-Verfahren rechnen, wie man es in semitischen Sprachen beobachten kann. ${ }^{100}$ Zu beachten ist dabei allerdings, dass im Ägyptischen die 3-rad. Verben nicht so dominant sind, wie sie dies in semitischen Sprachen sind. Dementsprechend könnten auch andere, stark belegte Verbalklassen eine eigene Formenbildung besessen haben. Zum anderen lässt sich trotz aller Schwäche der Schrift fallweise an den hieroglyphischen Graphien ablesen, dass ein und dieselbe paradigmatische Verbalform aus ein und derselben Verbalklasse bei verschiedenen Verben unterschiedlich gebildet werden kann, so bei Verben III.inf. der Prospektiv mit oder ohne die Endung $w .^{101}$ Man sollte sich also nicht der Illusion hingeben, dass die Bildung der finiten Verbalformen rasch abzuhandeln wäre. Man sollte aber dann auch alle Indizien nutzen, die die Texte selbst bieten, die Graphien, die Syntax und den Sinnzusammenhang, nicht zuletzt die Korrelationen zwischen Graphien, der Syntax und auch dem überlieferungsgeschichtlichen Zusammenhang. Dies, auch wenn kein Novum, so doch nicht allgemein akzeptiert, sei im folgenden an ausgewähltem Textmaterial expliziert.

Die Schreibung, schwacher" Konsonanten ist durchaus nicht regellos, jedenfalls in Textzeugen aus der Entstehungszeit eines Textes, bei denen lediglich an den einzelnen Textstellen mit Korruptelen zu rechnen ist. Während „schwache“ Konsonanten in bestimmten syntaktischen Umgebungen in aller Regel nicht auftreten, können sie in anderen Umgebungen nicht selten oder sogar häufig auftreten. Z.B. steht im älteren Ägyptisch nach der Partikel $i w$ in aller Regel endungsloses, ,śçm=f“. In den zum Zweck der Exemplifizierung herangezogenen Sargtexten sind die zahlreich belegten Verben ult. $i$ in diesem Fall

$99 \mathrm{Zu}$ einem minimalistischen Ansatz s. insbesondere Allen 2011a, Allen 2013, 111-19, und Allen 2014, zu einem maximalistischen Ansatz insbesondere Depuydt 2011.

100 Andréas Stauder verweist mich sehr zu Recht auf die Verhältnisse zumal des Akkadischen, die Kouwenberg 2010, § 2.3.1 darstellt.

101 Schenkel 2000a, 100-101. 


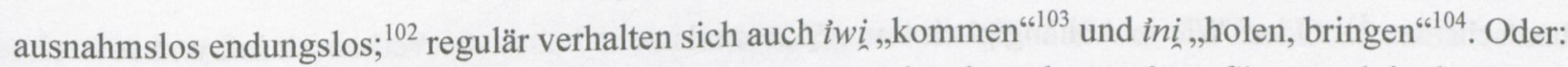
Nach der Negation $n n$ steht im älteren Ägyptisch in der Regel endungsloses ,śç $m=f^{\prime \prime}$, so auch in der Regel

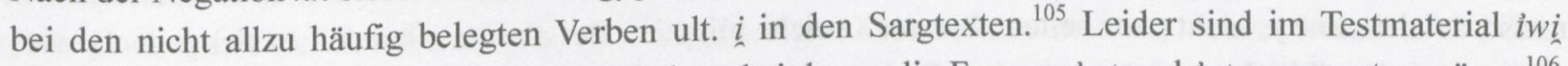
„,kommen“ und ini „,holen, bringen“ nicht belegt, bei denen die Formen iwt und int zu erwarten wären, ${ }^{106}$ die nach der Partikel iw nicht stehen können. (Nebenbei bemerkt: Ist dies so, ist, wie allgemein angenommen, das nach der Negation $n n$ gebrauchte endungslose ,śç̌m $=f “$, der Subjunktiv, von dem nach der Partikel $i w$ gebrauchten endungslosen,,$s ́ c ̧ m=f^{\prime \prime}$, der Umstandsform, verschieden.) Nicht ganz so einfach wie bei dem nach der Negation $n n$ oder bei dem nach der Partikel $i w$ gebrauchten ,śç̌m $=f$ " ist der Befund bei dem z.B. nach der Negation $n$ gebrauchten ,śç $m=f^{\prime \prime}$. Bei Verben eines Teils der Verbalklassen steht auch hier generell ein endungsloses ,śḉm=f“, so bei den starken 2-rad. und 3-rad. Verben und den II.red. ${ }^{107}$ In einem anderen Teil der Verbalklassen steht dagegen nicht selten oder häufig eine Endung $w$ oder $y$. Dies lässt sich, um beim zuvor gewählten Testmaterial zu bleiben, gut an den Verben ult. $i$ in den Sargtexten beobachten, so an den Belegen für $h$ 3i , herabsteigen“. ${ }^{108}$ Zur Exemplifizierung ausgewählt wurde gerade dieses Verb, weil man sich bei diesem in einen Widerspruch verstricken könnte. Belegt ist nämlich nicht nur die Kombination der Negation $n n$ mit einem endungslosen, „śç̌m=f“, sondern auch die Kombination mit einem ,śḉm=f“ mit einem zusätzlichen $y .{ }^{109}$ Pascal Vernus erklärt dies so: ${ }^{110}$ Es gab in ältester Zeit, wie James P. Allen das für die Pyramidentexte feststellt, zwei Konstruktionen mit der Negation $n, n s ́ c ̧ c m . w / y=f$ und $n \dot{s} c \grave{m}=f$. Im Verlauf der weiteren Sprachgeschichte wurde zuerst $n$ durch $n n$ ersetzt, es gab also

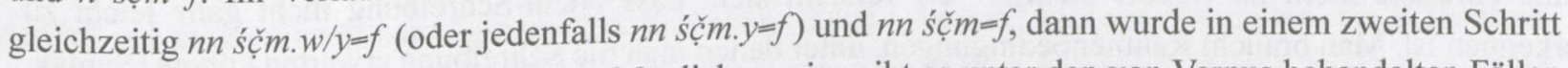

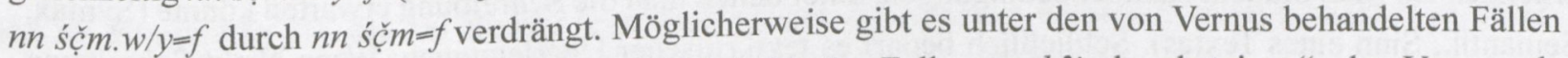
solche, in denen seine Erklärung unausweichlich ist. Im Falle von $h$, $i$,herabsteigen“, das Vernus als Paradebeispiel wählt und das auch in vorliegendem Zusammenhang im Zentrum des Interesses steht, ist dies nicht der Fall. In etwa der Hälfte der Handschriften ist die Negation $n$ durch die Negation $n n$ ersetzt, was die betreffenden Handschriften auch an anderen Textstellen einigermaßen regelmäßig tun. Dass sie nicht gleichzeitig die als śçm. $w / y=f$ (oder jedenfalls śç̌m. $y=f$ ) lesbare Graphie durch Streichung des $y$ auf

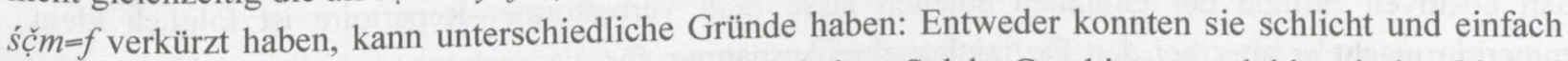
$h$;.) $y$ als eine alternative Graphie für $h y<h$ ? gehalten haben. Solche Graphien, pseudohistorisches $h$ ’ $y$ und realitätsnahes $h y$, sind auch belegt, zum mindesten in anderen Verbalformen und in anderen

102 Z.B. $i w=f i r=f$ (CT III, 293a; IV, 338l; VI, 273g, 343e); $i w=f h 3=f\left(\mathrm{CT} \mathrm{V}, 199 \mathrm{c}\right.$; VII, 262h); $i w$ Substantiv $h^{3}=f(\mathrm{CT}$ VI, 157g); $i w=f g r . t h 3=f$ (CT VII, 282e).

103 is $\breve{s m}$ / iw - (CT IV, 82d); unglaubwürdig iw iy- in einem oft problematischen Text neben $i w$ "kommen" ohne vorangehende Partikel $i w$ in weit zuverlässigeren Textzeugen (CT VI, 56j B2L).

104 iw=śn in=śn (CT VII, 45o).

$105 \operatorname{tr}$ (CT I, 60b, 189b), $m h$ (CT V, 176d), $h 3$ (CT IV, 305b, 323d; $h 3\{3\}$ IV, 305b M54C), hmś (CT IV, 306c, 323a, 323c, 324a); was in IV, $323 \mathrm{~d}$ T1Be wie $n n h \boldsymbol{\xi} . y=i$ aussieht, ist als Verschreibung eines $h \dot{\xi}=i$ zu erklären: das zweite Schilfblatt (M 17) von $y$ gehört eigentlich zur Graphie des Suffixpronomens $=i$, der Standardgraphie des Textzeugen, das erste Schilfblatt ist also zu problematisieren.

$106 \mathrm{Vgl}$. $n$ iwt - (Bauer B1, 57/alt=88/neu).

107 S. etwa die Übersichtstabellen bei Schenkel 2000a, 70 und 97.

108 Z.B. $n h 3=i / f$ (CT IV, 305b T2Be; VII, 413d B2Bo); $n h$ 3.w- (CT III, 48b B3C; IV, 305b B3C; V, 323d B3C; VII, 413d B1Bo B6C); $n h 3 . y=i / k f f$ (CT I, 189b B10C B12C; III, 48b B1C; IV, 305b B9C ${ }^{\text {b }}$ B9C $C^{\mathrm{a}}$ T1Be, 323d T1Be; VI, 288n B1Bo; VII, 413d B12C B9C); $n h\{\{3\}=i$ (CT IV, 305b B1Y); mehr bei Schenkel 2000a.

109 So $n n h$. $y=i$ (CT IV, 305b T1C ${ }^{\mathrm{b}} \mathrm{M} 4 \mathrm{C}$, wohl auch L1NY, 323d T1C ${ }^{\mathrm{b}}$ ).

110 Vernus 1990b, 121-24; ähnlich schon Loprieno 1986, 67-68. 


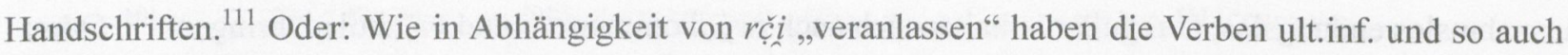
$h, i$,herabsteigen“ für ein und dasselbe ,śḉm=f“, den Subjunktiv, alternative Graphien bzw. Lautformen, (meist) solche ohne $y$, nicht ganz selten aber auch solche mit $y .{ }^{112}$. Bei diesem $y$ handelt es sich jedoch, wie andernorts plausibilisiert, nicht um eine Endung, sondern um einen irgendwie gearteten Reflex des „schwachen“ Radikals $i{ }^{1}{ }^{113}$ Bei der Aktualisierung des Textes durch Ersetzung der älteren Formulierung $n+$ Prospektiv durch die jüngere Formulierung $n n+$ Subjunktiv konnten die Schreiber wahlweise das als Endung zu verstehende $y$ des Prospektivs löschen oder als Reflex des „,schwachen“ Radikals $i$ im Subjunktiv stehen lassen.

Was die Aufdeckungsprozedur angeht, ist so zu verallgemeinern: $\mathrm{Zu}$ unterscheiden ist nicht zwischen Fällen, in denen eine Endung aus einem ,schwachen“ Konsonanten geschrieben ist, und Fällen, in denen eine solche Endung nicht geschrieben ist, sondern zwischen Fällen, in denen eine Endung aus einem „Schwachen“ Konsonanten geschrieben sein kann, und Fällen, in denen sie in aller Regel nicht geschrieben ist. Dies ergibt sich einfach daraus, dass „schwache“ Konsonanten geschrieben werden können, aber nicht geschrieben werden müssen. Ein klassisches Beispiel für einen Fehlschluss, der auf der Missachtung dieser Regel beruht, ist Elmar Edels , $s \underline{d} m w f^{\prime \prime}$, das den heutigen Prospektiv mit den Vorkommen erfasst, in denen ein "schwacher" Konsonant geschrieben ist (oder in Parallelen zu der betreffenden Textstelle geschrieben ist), dagegen die Vorkommen übersieht, in denen ein „schwacher" Konsonant nicht geschrieben ist (bzw. eine Parallele nicht zu Gebote steht). ${ }^{114}$ Es versteht sich, dass Nicht-Schreibung nicht ganz leicht zu erkennen ist. Man braucht Rahmenbedingungen, unter denen man die Schreibung erwarten könnte (Syntax, Semantik, Sinn eines Textes). Schließlich bedarf es textkritischer Überlegungen: Wenn Nicht-Schreibung in einem Fall die generelle Regel ist, kann an der einzelnen Textstelle irrtümlich auch einmal geschrieben sein, was eigentlich nicht geschrieben sein sollte.

Sami Uljas erinnert an die unterschiedlichen Positionen, die Kurt Sethe und Adolf Erman Ende des 19. und zu Beginn des 20. Jahrhunderts in solchen Fragen bezogen. ${ }^{115}$ Für Sethe ist nur relevant, was sich aus dem positiven Befund der Graphien ableiten lässt. Sein Verbalformen-Repertoire ist folglich klein. Immerhin macht er aber bei den Partizipien eine Ausnahme, für die er durchgängig einen Unterschied zwischen perfektischen und imperfektischen gelten lässt, also auch in Verbalklassen, die einen solchen Unterschied in den Graphien - Reduplikation vs. Nicht-Reduplikation - nicht erkennen lassen. Heutzutage müsste man das Sethe-Repertoire auch darüber hinaus noch etwas erweitern, nämlich mit dem śç $m$. $w=f$ in der Graphien-basierten Version, die Edel in die Diskussion einbrachte und von der gerade die Rede war. Erman dagegen spricht im Prinzip allen Verben, zu welcher Verbalklasse sie auch gehören mögen, ein gleichartiges Flexionsformen-Repertoire zu. Unterschiede, die nur in einem Teil der Verbalklassen zu beobachten sind, werden analog auf alle anderen Verbalklassen übertragen. Uljas, der Sicherheit zu gewinnen sucht und daher Analogieschlüssen gegenüber zurückhaltend ist, wäre es lieber, wenn man sich mit Sethes Position zufrieden geben könnte. Dem sind aber die hier eingangs behandelten Gegebenheiten beim Infinitiv, beim Pseudopartizip und bei den Partizipien entgegen zu halten. Die Formenbildung ist tatsächlich wesentlich komplizierter als dies die Graphien zu erkennen geben. Es zeigen sich, wie oben demonstriert,

111 Imperativ: $h 3 y$ (CT V, 167d A1C, 254d S14C), hy (CT V, 167d G1T, 254d B4Bo B6C S14C u.ä.); śçm.t=f: h3̉y.t (CT V, 2031 M1C, 207c M1C).

112 Schenkel 2000a, 62-64.

113 Schenkel 2000a, 65-69.

114 Edel 1955-64, §§ 511-31; Edel 1959, 108-11; Edel 1984a.

115 Uljas 2008, s. auch Uljas 2011. 
in der Vokalisation sogar Unterschiede, die für die paradigmatische Einordnung der Flexionsformen keine Rolle spielen. Andererseits werden nicht in allen Verbalklassen miteinander korrespondierende Formen gebildet. Erinnert sei an die Infinitive zweiradikaliger Verben, die mit nur einem Vokal nicht die Unterschiede realisieren können, die bei drei- und mehrradikaligen Verben zu beobachten sind. Die Waagschale neigt sich eindeutig zu Ermans Seite, eher noch tiefer als Erman dachte.

Man kann sich die ägyptische paradigmatische Realität ganz gut vorstellen, wenn man das deutsche Verbalparadigma daneben hält. Jedes deutsche Verb bildet einen Infinitiv, ein Präteritum und ein Partizip Perfekt. Die Formenbildung ist aber nicht einheitlich. Ein Teil der Verben bildet Formen nach der schwachen Konjugation, ein anderer Teil nach der starken Konjugation und in letzterem Falle auch noch von Verb zu Verb vielfach verschieden:

\begin{tabular}{|l|l|l|l|}
\hline Konjugation & Infinitiv & Präteritum & Partizip Perfekt \\
\hline schwache & sagen & sagte & gesagt \\
\hline \multirow{3}{*}{ starke } & singen & sang & gesungen \\
\cline { 2 - 4 } & brechen & brach & gebrochen \\
\hline
\end{tabular}

Diachronisch gibt es Änderungen in der Besetzung der Positionen. Im folgenden, um die paradigmatische Position Konjunktiv II erweiterten Beispiel sei veranschaulicht die Ersetzung des Präteritums „,buk“ durch „backte“ und des Konjunktivs II „büke“ ebenfalls durch „,backte“, was temporär zum lautlichem Zusammenfall unterschiedlicher paradigmatischer Positionen führt (,,backte“ in zwei Positionen, als Präteritum und als Konjunktiv II), die bei Bedarf aber auch wieder differenziert werden können (,,backte“ in einer Position erhalten, in der anderen ersetzt durch die Umschreibung ,würde backen“):

\begin{tabular}{|l|l|l|l|l|}
\hline & Infinitiv & Präteritum & Konjunktiv II & Partizip Perfekt \\
\hline \multirow{2}{*}{ Zustand I } & \multirow{4}{*}{ backen } & buk & büke & \\
\cline { 3 - 4 } Zustand II & backte & backte & \multirow{3}{*}{ gebacken } \\
\cline { 3 - 4 } Zustand III & & würde backen & \\
\hline
\end{tabular}

Es kann sich aber diachronisch auch die Struktur des Paradigmas selbst ändern, vgl. dazu den (weitgehenden) Verlust der Spalte „Präteritum“ in süddeutschen Dialekten (die Lautformen vereinfachend in hochsprachliche Lautformen übersetzt):

\begin{tabular}{|l|l|l|l|l|}
\hline $\begin{array}{l}\text { Sprachvarietät, } \\
\text { Konjugation }\end{array}$ & Infinitiv & Präteritum & Konjunktiv II & Partizip Perfekt \\
\hline Hochsprache, stark & backen & backte & $\begin{array}{l}\text { backte } \\
\text { würde backen }\end{array}$ & gebacken \\
\hline Dialekt, schwach & backen & - & täte backen & gebackt \\
\hline
\end{tabular}

Zurück zum Ägyptischen: Man kann zwar bei gut belegten Verben der Realität nahe kommen. Die Masse der Verben ist aber zu schwach belegt, als dass man sich hier allzu großen Erwartungen hingeben könnte. Es fehlt an Graphien und es fehlt erst recht an Indizien für die Vokalisation. Man kann zwar eine Vorstellung davon gewinnen, wie die Realität im Prinzip aussah, man kann sie aber nur sehr partiell konkret rekonstruieren. Man kann möglicherweise ein Paradigma rekonstruieren, auch wenn man die Einzelformen nicht rekonstruieren kann. So dürfte auch die Belegmasse dazu ausreichen, paradigmatische Veränderungen, wie sie an deutschen Dialekten gerade veranschaulicht wurden, zu erkennen. So kann es z.B. keinen Zweifel daran geben, dass im Laufe der älteren ägyptischen Sprachgeschichte die paradigmatische Position „Prospektiv" verloren ging. 


\section{BIBLIOGRAPHY AND TEXT SOURCES}

Adamson, Sylvia, and Wendy Ayre-Bennett

2011 "Linguistics and Philology in the Twenty-First Century: Introduction," Transactions of the Philological Society 109.3, 201-206.

Allen, James P.

1984 The Inflection of the Verb in the Pyramid Texts. Bibliotheca Aegyptia 2. Malibu.

1991 "Form, Function and Meaning in the Early Egyptian Verb," Lingua Aegyptia 1, 1-32.

1994 "Pronominal Rhematization," in David P. Silverman, ed., For His Ka: Essays Offered in Memory of Klaus Baer (Studies in Ancient Oriental Civilization 55; Chicago), 1-13.

2000 Middle Egyptian: an Introduction to the Language and Culture of Hieroglyphs. Cambridge.

2002 The Heqanakht Papyri. Publications of the Metropolitan Museum of Art Egyptian Expedition 27. New York.

2004 "Traits dialectaux dans les Textes des Pyramides du Moyen Empire," in Susanne Bickel and Bernard Mathieu, ed., D'un monde à l'autre: Textes des Pyramides et Textes des Sarcophages, actes de la table ronde internationale "Textes des Pyramides versus Textes des Sarcophages, " IFAO 24-26 septembre 2001 (Bibliothèque d'étude 139; Cairo), 1-14.

2005 The Ancient Egyptian Pyramid Texts, ed. by Peter Der Manuelian. Writings from the Ancient World 23. Atlanta and Boston.

2006 "Some Aspects of the Non-royal Afterlife in the Old Kingdom," in Miroslav Bárta, ed., The Old Kingdom Art and Archaeology. Proceedings of the Conference Held in Prague, May 31 - June 4, 2004 (Prague), 9-18.

2010 Middle Egyptian: an Introduction to the Language and Culture of Hieroglyphs, 2 nd ed. Cambridge.

2011 a "Rethinking the $s \underline{d m} . f$ " Lingua Aegyptia 19, 1-16.

2011b The Debate Between a Man and his Soul: a Masterpiece of Ancient Egyptian Literature. Culture and History of the Ancient Near East 44. Leiden.

2013 The Ancient Egyptian Language, an Historical Study. Cambridge.

2014 "Emphatic Sentences and Nominal/Relative Forms in Earlier Egyptian," Lingua Aegyptia 21, 1-7.

2015 Middle Egyptian Literature: Eight Literary Works of the Middle Kingdom. Cambridge.

Altenmüller, Hartwig

1998 Die Wanddarstellungen im Grab des Mehu in Saqqara. Archäologische Veröffentlichungen, Deutsches Archäologisches Institut, Kairo 42. Mainz.

2011 "Reden und Rufe auf zwei Gräberbilddern des Alten Reiches," in Frank Feder et al., ed., Von Theben nach Giza: Festmiszellen für Stefan Grunert zum 65. Geburtstag (Göttinger Miszellen, Beihefte 10; Göttingen), 19-22.

Amenemhet Wolfgang Helck. Der Text der "Lehre Amenemhets I. für seinen Sohn." Kleine Ägyptische Texte 1. Wiesbaden, 1969.

Anthes, Rudolf

1930 Eine Polizeistreife des Mittleren Reiches in die westliche Oase," Zeitschrift für Ägyptische Sprache und Altertumskunde 65, 108-14.

Arnott, D. W.

1970 The Nominal and Verbal System of Fula. Oxford.

Assmann, Jan

1997 Das Kulturelle Gedächtnis: Schrift, Erinnerung und politische Identität in frühen Hochkulturen, Munich. 
(Assmann, Jan)

2002 Altägyptische Totenliturgien I: Totenliturgien in den Sargtexten des Mittleren Reiches. Supplemente zu den Schriften der Heidelberger Akademie der Wissenschaften, Philosophisch-historische Klasse, 14. Heidelberg.

Badawy, Alexander

1976 The Tombs of Iteti, Sekhem'ankh-Ptah and Kaemnofert at Giza. University of California Publications: Occasional Papers 9: Archaeology. Los Angeles.

Baer, Klaus

1966 "A deed of endowment in a letter of the time of Ppjj I?," Zeitschrift für Ägyptische Sprache und Altertumskunde 93, 1-9.

Baerman, Matthew

2004 "Directionality and (Un)natural Classes in Syncretism," Language 80, 807-27.

Baerman, Matthew, Dunstan Brown, and Greville G. Corbett

2005 The Morphology-Syntax Interface: A Study of Syncretism. Cambridge Studies in Linguistics 109. Cambridge.

Baker, Mark

1985 "The Mirror Principle and Morphosyntactic Explanation," Linguistic Inquiry 16, 373-415.

Bakir, Abd el-Mohsen

1970 Egyptian Epistolography from the Eighteenth to the Twenty-first Dynasty. Bibliothèque d'étude 48. Cairo.

Barbash, Yekaterina

2011 The Mortuary Papyrus of Padikakem. Walters Art Museum 551. Yale Egyptological Studies 8. New Haven. Barber, Peter J.

2013 Sievers' Law and the History of Semivowel Syllabicity in Indo-European and Ancient Greek. Oxford Classical Monographs. Oxford.

Barns, John W. B.

1956 Five Ramesseum Papyri. Oxford.

Baud, Michel and Dominique Farout

2001 "Trois biographies d'Ancien Empire revisitées," Bulletin de l'Institut Français d'Archéologie Orientale 101, 43-57.

Bauer see Peas.

BD Nu Günther Lapp, Catalogue of Books of the Dead in the British Museum, I. The Papyrus of Nu (BM EA 10477). London, 1997.

Beaver, David, and Cleo Condoravdi

2003 "A Uniform Analysis of before and after," in Robert B. Young and Yuping Zhou, ed., Proceedings from Semantics and Linguistics Theory 13 (SALT) (Ithaca), 37-57.

Bendjaballah, Sabrina and Chris H. Reintges

2009 "Ancient Egyptian Verbal Reduplication: Typology, Diachrony, and the Morphology-Syntax Interface," in Bernhard Hurch and Veronika Mattes, ed., Special Issue on Reduplication: Diachrony and Productivity (Morphology 19), 135-57.

Beni Hasan Percy E. Newberry, Beni Hasan. 4 vols. Egypt Exploration Fund 1-2, 5, 7. London, 1893-1900.

Berlin

1911 Hieratische Papyrus aus dem Königlichen Museen zu Berlin III: Schriftstücke der VI. Dynastie aus Elephantine; Zaubersprüche für Mutter und Kind; Ostraka. Leipzig, 1911.

Boeser, Pieter

1909 Beschrijving van de Egyptische Verzameling in het Rijksmuseum van Oudheden te Leiden, II. Den Haag. 
Bolinger, Dwight

1977 The Form of Language. London.

1985 "The Inherent Iconism of Intonation," in John Haiman, ed., Iconicity in Syntax (Typological Studies in Language 6; Amsterdam and Philadelphia), 97-108.

Borghouts, Joris

1989 "Aspectual Values of the Second Tenses in Middle Egyptian," in Silvia Schoske et al., ed., Linguistik, Philologie, Religion. Akten des vierten Internationalen Ägyptologen-Kongresses, München 1985 (Studien zur Altägyptischen Kultur, Beihefte 3; Hamburg), 29-42.

2010 Egyptian, an Introduction to the Writing and Language of the Middle Kingdom 1: Grammar, Syntax and Indexes. Egyptologische Uitgaven 24. Leuven and Leiden.

Bresnan, Joan, and Sam A. Mchombo

1987 "Topic, Pronoun, and Agreement in Chichewa," Language 63, 741-82.

Brose, Marc

2014 Grammatik der dokumentarischen Texte des Mittleren Reiches. Lingua Aegyptia Studia Monographica 13. Hamburg.

Brovarski, Edward J.

2001 Giza Mastabas, 7. The Senedjemib Complex, I: The Mastabas of Senedjemib Inti (G 2370), Khnumenti (G 2374), and Senedjemib Mehi (G 2378), 2 vols. Boston.

Buchberger, Hannes

1995 "Das Harfnerlied im Grab $k$; $(=i)-m$ - $n h$ oder 'Die Riten des $s n n t r w$ '," in Dieter Kessler and Regine Schulz, ed., Gedenkschrift für Winfried Barta: htp dj $n$ h̆jj (Münchener Ägyptologische Untersuchungen 4; Frankfurt am Main), 93-123.

Butt, John, and Carmen Benjamin

2011 A New Reference Grammar of Modern Spanish, 4th ed. London.

Bybee, Joan L.

1985 "Diagrammatic iconicity in stem-inflection relations," in John Haiman, ed., Iconicity in Syntax (Typological Studies in Language 6; Amsterdam and Philadelphia), 11-48.

2010 Language, Usage, and Cognition. Cambridge.

Cairo CG 1-1294: Ludwig Borchardt, Statuen und Statuetten von Königen und Privatleuten im Museum zu Kairo, Nr. 1-1294. Catalogue général des antiquités égyptiennes du Musée du Caire. Berlin, 1911-36.

20001-20780: Hans O. Lange and Heinrich Schaefer, Grab- und Denksteine des Mittleren Reichs, 4 vols. Catalogue général des antiquités égyptiennes du Musée du Caire 51-54. Berlin, 1902-25.

Cannuyer, Christian

1990 "Recherches sur l'onomasiologie du feu," Zeitschrift für Ägyptische Sprache und Altertumskunde 117, 103-11.

Carnarvon, George E. S. M. H., Earl of, and Howard E. Carter

1912 Five Years' Exploration at Thebes: a Record of Work Done 1907-1911. London and New York.

Chafe, Wallace

1994 Discourse, Consciousness, and Time: the Flow and Displacement of Conscious Experience in Speaking and Writing. Chicago.

1995 "The Realis-Irrealis Distinction in Caddo, the Northern Iroquoian Languages, and English," in Joan L. Bybee and Suzanne Fleischman, ed., Modality in Grammar and Discourse. (Typological Studies in Language 32; Amsterdam and Philadelphia), 349-65.

Cherpion, Nadine, Jean-Pierre Corteggiani, and Jean-François Gout

2007 Le tombeau de Pétosiris à Touna el-Gebel. Bibliothèque générale 27. Cairo. 


\section{Chierchia, Gennaro}

2013 Logic in Grammar. Polarity, Free Choice, and Intervention. Oxford Studies in Semantics and Pragmatics 2. Oxford.

\section{Chomsky, Noam}

1981 Lectures on Government and Binding: The Pisa Lectures. Studies in Generative Grammar 9. Dordrecht.

1995 The Minimalist Program. Current Studies in Linguistics 28. Cambridge, MA.

2001 "Derivation by Phase," in Michael Kenstowicz, ed., Ken Hale: a Life in Linguistics (Current Studies in Linguistics 36; Cambridge, MA), 1-52.

\section{Cinque, Guglielmo}

1999 Adverbs and Functional Heads, a Cross-Linguistic Perspective. Oxford Studies in Comparative Syntax. Oxford.

Clère, Jacques Jean

1995 Les chauves d'Hathor. Orientalia Lovaniensia Analecta 63. Louvain.

Collier, Mark A.

1990 "The Circumstantial $s \underline{d} m(. f) / s \underline{d} m . n(f)$ as Verbal Verb-Forms in Middle Egyptian," Journal of Egyptian Archaeology 76, 73-85.

1991 "Circumstantially Adverbial? The Circumstantial $s \underline{d} m(. f) / s \underline{d} m . n(. f)$ Reconsidered," in Stephen Quirke, ed., Middle Kingdom Studies (New Malden), 21-50.

1992 "Predication and the Circumstantial $s \underline{d} m(. f) / s \underline{d}$ dm.n.ff)," Lingua Aegyptia 2, 17-65.

1994 "Grounding, Cognition and Metaphor in the Grammar of Middle Egyptian: the Role of Human Experience in Grammar as an Alternative to the Standard Theory Notion of Paradigmatic Substitution," Lingua Aegyptia 4, 57-87.

Collier, Mark A., and Stephen Quirke

2002 The UCL Lahun Papyri: Letters. BAR International Series 1083. London.

Collombert, Philippe, and Laurent Coulon

2000 "Les dieux contre la mer: le début du 'papyrus d'Astarté' (pBN 202)," Bulletin de l'Institut Français d'Archéologie Orientale 100, 193-242.

Comrie, Bernard

1976 Aspect: an Introduction to the Study of Verbal Aspect and Related Problems (Cambridge Textbooks in Linguistics). Cambridge.

1977 Tense. Cambridge Textbooks in Linguistics. Cambridge.

1982 "Aspect and Voice: Some Reflections on Perfect and Passive," in Philip Tedeschi and Annie Zaenen, ed., Tense and Aspect (Syntax and Semantics 14; New York), 65-78.

Corbett, Greville G.

2006 Agreement. Cambridge Textbooks in Linguistics. Cambridge.

Croft, William

1996 "What's a Head?," in Johan Rooryck and Laurie Zaring, ed., Phrase Structure and the Lexicon (Studies in Natural Language and Linguistic Theory 33; Dordrecht), 35-75.

2001 Radical Construction Grammar: Syntactic Theory in Typological Perspective. Oxford.

CT

I-VII Adriaan de Buck, The Egyptian Coffin Texts, 7 vols. Oriental Institute Publication 34, 49, 64, 67, 72, 81, 87. Chicago, 1935-61.

VIII James P. Allen, The Egyptian Coffin Texts, VIII: Middle Kingdom Copies of Pyramid Texts. Oriental Institute Publications 132. Chicago, 2006.

Cysouw, Michael

2003 The Paradigmatic Structure of Person Marking. Oxford. 
2010 “Semantic Maps as Metrics on Meaning," Linguistic Discovery 8, 70-95.

Czerwik, Dorota

1999 "Some Remarks on the Letters to the Dead from the First Intermediate Period," Göttinger Miszellen 173, 61-66.

Dahl, Östen

1979 "Typology of Sentence Negation," Linguistics 17, 79-106.

1985 Tense and Aspect Systems. Oxford.

David, Arlette

2006 Syntactic and Lexico-Semantic Aspects of the Legal Register in Ramesside Royal Decrees. Göttinger Orientforschungen IV, 38. Wiesbaden.

Davies, Norman de Garis

1943 The Tomb of Rekh-mi-Re' at Thebes, 2 vols. Publications of the Metropolitan Museum of Art Egyptian Expedition 11. New York.

Depuydt, Leo

1986 "The Emphatic Nominal Sentence in Egyptian and Coptic," in Gerie Englund and Paul J. Frandsen, ed., Crossroad: Chaos or the Beginning of a New Paradigm. Papers from the Conference on Egyptian Grammar, Helsingør 28-30 May 1986 (Copenhagen), 91-117.

1988 "New Horizons in Coptic and Egyptian Linguistics," in Chronique d'Égypte 63 (126), 391-406.

1991 "On Distinctive and Isolating Emphasis in Egyptian and in General," Lingua Aegyptia 1, 33-56.

2008a The Other Mathematics: Language and Logic in Egyptian and in General. Piscataway, NJ.

2008b "Zum Nebeneinander von An- und Abwesenheit der Gemination in der Wendung mrjj jt.f mrrw snw.f," Lingua Aegyptia 16, 27-38.

2011 "Zu Lehr- und Lernbarkeit des ägyptischen Verbs: Wie viele typisch mittelägyptische $s \underline{d m}$.f-Formen gibt es eigentlich? Neun!," in Alexandra Verbovsek et al., ed., Methodik und Didaktik in der Ägyptologie: Herausforderungen eines kulturwissenschaftlichen Paradigmenwechsels in den Altertumswissenschaften. (Ägyptologie und Kulturwissenschaft 4; München), 481-508.

Derchain-Urtel, Maria-Theresa

1973 "Das n-Präfix im Ägyptischen," Göttinger Miszellen 6, 39-54.

Desclaux, Vanessa

2014 "Les appels aux passants en Égypte ancienne: approche historique d'un genre littéraire," 2 vols. Thèse de doctorat, Université de Lyon II.

Di Biase Dyson, Camilla

2012 "A Diachronic Approach to the Syntax and Semantics of Egyptian Spatio-temporal Expressions with $h$ $3-t$ 'front': Implications for Cognition and Metaphor," in Eitan Grossman et al., ed., Lexical Semantics in Ancient Egyptian (Lingua Aegyptia Studia Monographica 9; Hamburg), 247-92.

Di Biase Dyson, Camilla, Frank Kammerzell, and Daniel Werning

2009 "Glossing Ancient Egyptian: Suggestions for Adapting the Leipzig Glossing Rules," Lingua Aegyptia $17,343-66$.

Díaz Hernández, Roberto A.

2013 Tradition und Innovation in der offiziellen Sprache des Mittleren Reiches: ein strukturalistischer Vergleich der historisch-biographischen mit den literarischen Texten in der 1. Zwischenzeit und der 12. Dynastie. Göttinger Orientforschungen IV, 56. Wiesbaden.

Dixon, Robert M. W.

2004 "Adjective Classes in Typological Perspective," in Robert M. W. Dixon und Alexandra Y. Aikhenwald, ed., Adjective Classes: a Cross-Linguistic Typology (Oxford), 1-49. 
Donnat Beauquier, Sylvie

2009 "Le rite comme seul référent dans les lettres aux mort," Bulletin de l'Institut Français d'Archéologie Orientale 109, 61-93.

2014 Écrire à ses morts: enquête sur un usage rituel de l'écrit dans l'Égypte pharaonique. Grenoble.

Doret, Éric

1986 The Narrative Verbal System of Old and Middle Egyptian. Cahiers d'Orientalisme 12. Genève.

1994 "Ankhtifi and the Description of his Tomb in Mo'alla," in David P. Silverman, ed., For His Ka: Essays Offered in Memory of Klaus Baer (Studies in Ancient Oriental Civilization 55; Chicago), 79-86.

Doron, Edit

1988 "On the Complementarity of Subject and Subject-Verb Agreement," in Michael Barlow and Charles A. Ferguson, ed., Agreement in Natural Language: Approaches, Theories, Descriptions: Papers Presented at a Conference held at Stanford University in October 1984 (Stanford), 201-18.

1999 "V-Movement and VP Ellipsis," in Shalom Lappin and Elabbas Benmamoun, ed., Fragments: Studies in Ellipsis and Gapping (Oxford), 124-40.

Doron, Edit, and Caroline Heycock

2010 "In Support of Broad Subjects in Hebrew," Lingua 120, 1764-76.

Drioton, Étienne

1943 "Description sommaire des chapelles funéraires de la VIe dynastie récemment découvertes derrrière le mastaba de Mérérouka à Sakkarah," Annales du Service des Antiquités de l'Égypte 43, 487-513.

Drioton, Étienne, and Jean-Philippe Lauer

1958 "Un groupe de tombes à Saqqarah: Icheti, Nefer-khouou-Ptah, Sébek-em-khent et Ankhi," Annales du Service des Antiquités de l'Égypte 55, 207-51.

Dryer, Matthew S.

2013 "Position of Tense-Aspect Affixes," in Mathew S. Dryer and Martin Haspelmath, ed., The World Atlas of Language Structures Online (Leipzig), http://wals.info/chapter/69 (accessed 05/09/2014).

Dubinsky, Stanley, and Silvester R. Simango

1996 "Passive and Stative in Chichewa: Evidence for Modular Distinctions in Grammar," Language 72, 74981.

Dunham, Dows

1937 The Naga-ed-Dêr Stelae of the First Intermediate Period. London.

DZA See TLA

Edel, Elmar

1955-64 Altägyptische Grammatik. Analecta Orientalia 34/39. Roma.

1959 "Beiträge zur ägyptischen Grammatik," Zeitschrift für ägyptische Sprache und Altertumskunde 84, 10513.

1973 "Unsere Kenntnis des altägyptischen," in Serge Sauneron, ed., Textee et langages de l'Égypte pharaonique: cent cinquante années de recherches 1822-1972. Hommages à Jean-François Champollion, 3 vols. (Bibliothèque d'étude 64; Cairo), I, 119-23.

1980 Neue Deutungen keilschriftlicher Umschreibungen ägyptischer Wörter und Personennamen. Sitzungsberichte der Österreichischen Akademie der Wissenschaften 375. Wien.

1981 Hieroglyphische Inschriften des Alten Reiches. Abhandlungen der Rheinisch-Westfälischen Akademie der Wissenschaften 67. Opladen.

1984a "Neue Belege für die aktivische $s d m w . f$-Form," in Friedrich Junge, ed., Studien zu Sprache und Religion Ägyptens zu Ehren von Wolfhart Westendorf überreicht von seinen Freunden und Schülern (Göttingen), 25-39. 
1984b Die Inschriften der Grabfronten der Siut-Gräber in Mittelägypten aus der Herakleopolitenzeit, eine Wiederherstellung nach den Zeichnungen der Description de l'Égypte. Abhandlungen der RheinischWestfälischen Akademie der Wissenschaften 71. Opladen.

1994 "Der vollständige Architrav vom Grab des mḥj in Saqqara," in Betsy M. Bryan and David Lorton, ed., Essays in Egyptology in Honor of Hans Goedicke (San Antonio), 61-67.

Eichler, Eckhard

1991 "Untersuchungen zu den Königsbriefen des Alten Reiches," in Studien zur altägyptischen Kultur 18, 141-71.

el-Aguizy, Ola

2010 "Une nouvelle 'tombe-sarcophage à puits' à Saqqara," Bulletin de l'Institut Français d'Archéologie Orientale 110, 13-34.

el-Awady, Tarek

2009 Abusir, XVI. Sahure - the Pyramid Causeway: History and Decoration Program in the Old Kingdom. Prague.

el-Hamrawi, Mahmoud

2004a "Alte-Reichs-Sprache und Mittlere-Reichs-Sprache in abydenischen Texten der 11.-12. Dynastie," Lingua Aegyptia 12, 89-122.

2004b "Subtantiv + is im Altägyptichen," in Jean-Claude Goyon and Christine Cardin, ed., Proceedings of the Ninth International Congress of Egyptologists: Grenoble, 6-12 septembre 2004 (Orientalia Lovaniensia Analecta 150; Leuven), I, 545-65.

el-Khouli, Ali, and Naguib Kanawati

1988 Excavations at Saqqara, North-West of Teti's Pyramid II. Sydney.

Elliott, Jennifer R.

2000 "Realis and Irrealis: Forms and Concepts of the Grammaticalisation of Reality," Linguistic Typology 4, $55-90$.

Embick, David

2004 "On the Structure of Resultative Participles in English," Linguistic Inquiry 35, 355-92.

Emonds, Joseph

1978 "The verbal complex V'-V in French," Linguistic Inquiry 9, 151-75.

Englund, Gertie, und Paul John Frandsen

1986 ed., Crossroad: Chaos or the Beginning of a New Paradigm. Papers from the Conference on Egyptian Grammar. Helsingør 28-30 May 1986. Copenhagen.

Engsheden, Åke

2003 La reconstitution du verbe en égyptien de tradition 400-30 avant J.-C. Uppsala Studies in Egyptology 3. Uppsala.

Erman, Adolf

1889 Die Sprache des Papyrus Westcar. Mittheilungen der Königlichen Gesellschaft der Wissenschaften zu Göttingen. Göttingen.

1894 Ägyptische Grammatik mit Schrifttafel, Litteratur, Lesestücken und Wörterverzeichnis, 1st ed. Porta Linguarum Orientalium 15. Berlin.

1919 Reden, Rufe und Lieder aufGräberbildern des alten Reiches. Abhandlungen der Preussischen Akademie der Wissenschaften, Philosophisch-historische Klasse 15. Berlin.

1928 Ägyptische Grammatik, mit Schrifttafel, Paradigmen und Übungstücken zum Selbststudium und zum Gebrauch in Vorlesungen. Porta Linguarum Orientalium 15. Berlin.

Eyre, Christopher J.

2013 The Use of Documents in Pharaonic Egypt. Oxford Studies in Ancient Documents. Oxford. 
Fălăuş, Anamaria

2013 "Introduction: Alternatives in Semantics and Pragmatics," in Anamaria Fălăuş, ed., Alternatives in Semantics (Palgrave Studies in Pragmatics, Language and Cognition; Basingstoke), 1-35.

Farout, Dominique

2012 "Les déclarations du roi Sahourê," Revue d'Égyptologie 63, 103-14.

2013 "Naissance du dialogue de cour sur les monuments d'Ancien Empire," Revue d'Égyptologie 64, 15-23.

Faulkner, Raymond O.

1969 The Ancient Egyptian Pyramid Texts Translated into English, 2 vols. Oxford.

1973-78 The Ancient Egyptian Coffin Texts, 3 vols. Warminster.

1976 "The Enclitic Negation w," Journal of Egyptian Archaeology 62, 176.

1982 “A Coffin Text Miscellany," Journal of Egyptian Archaeology 68, 27-30.

Fecht, Gerhard

1960 Wortakzent und Silbenstruktur. Untersuchungen zur Geschichte der ägyptischen Sprache. Ägyptologische Forschungen 21. Glückstadt-Hamburg-New York.

Firchow, Otto

1954 "Zu den Wortverbindungen mit ś.t," Zeitschrift für Ägyptische Sprache und Altertumskunde 79, 91-94.

Fischer, Henry G.

1977 Egyptian Studies II. The Orientation of Hieroglyphs, Part 1: Reversals. New York.

1991 "Marginalia," Göttinger Miszellen 122, 12-30.

1996 Egyptian Studies III: Varia Nova. New York.

Fischer-Elfert, Hans-Werner, and Alfred Grimm

2003 "Autobiographie und Apotheose: Die Statue des Žs(š)n Z3-Hw.t-Hrw im Staatlichen Museum Ägyptischer Kunst München," Zeitschrift für Ägyptische Sprache und Altertumskunde 130, 60-80.

Fowler Richard B. Parkinson, "The Discourse of the Fowler: Papyrus Butler verso (P. BM EA 10274)," Journal of Egyptian Archaeology 90 (2004), 81-111.

François, Alexandre

2008 "Semantic Maps and the Typology of Colexification: Intertwining Polysemous Networks across Languages," in Martine Vanhove, ed., From Polysemy to Semantic Change: Towards a Typology of Lexical Semantic Associations (Amsterdam), 163-215.

Freed, Rita E.

1996 "Stela Workshops of Early Dynasty 12," in Peter Der Manuelian, ed., Studies in Honor of William Kelly Simpson (2 vols.; Boston), I, 297-336.

Frood, Elizabeth

2007 Biographical texts from Ramessid Egypt. Writings from the Ancient World 26. Atlanta, 2007.

Fukui, Naoki

1993 "Parameters and Optionality," Linguistic Inquiry 24, 399-420.

Fukui, Naoki, and Margaret Speas

1986 "Specifiers and Projection," in Naoki Fukui et al., ed., Papers in Theoretical Linguistics (MIT Working Papers in Linguistics 8; Cambridge, MA), 128-72.

Gardiner, Alan H.

1909 The Admonitions of an Egyptian Sage from a Hieratic Papyrus in Leiden (Pap. Leiden 344 Recto). Leipzig.

1927 Egyptian Grammar. Being an Introduction to the Study of Hieroglyphs. Oxford.

1930 "A New Letter to the Dead," Journal of Egyptian Archaeology 16, 19-22.

1932 The Theory of Speach and Language. Oxford.

1935 Hieratic Papyri in the British Museum, Third Series, Chester Beatty Gift, 2 vols. London. 
1947a Ancient Egyptian Onomastica, 3 vols. Oxford.

1947b Review of Études de syntaxe copte by H. J. Polotsky, Journal of Egyptian Archaeology 33, 95-101.

1948 "The First Two Pages of the 'Wörterbuch'," Journal of Egyptian Archaeology 34, 12-18.

1950 Egyptian Grammar. Being an Introduction to the Study of Hieroglyphs. 2nd ed. Oxford.

1957 Egyptian Grammar. Being an Introduction to the Study of Hieroglyphs. 3rd ed. Oxford.

1962 My Working Years. Private printing.

Gardiner, Alan H., and Kurt Sethe

1928 Egyptian Letters to the Dead, Mainly from the Old and Middle Kingdoms. London.

Gemnikai Friedrich Wilhelm von Bissing, Die Mastaba des Gem-ni-kai, 2 vols. Berlin.

Gestermann, Louise

1994 “Neue' Texte in spätzeitlichen Grabenanlagen von Saqqara und Heliopolis," in Martina Minas and Jürgen Zeidler, ed., Aspekte spätägyptischen Kultur: Festschrift für Erich Winter zum 65. Geburtstag (Aegyptiaca Treverensia 7; Mainz), 89-95.

Gilula, Mordechai

1970 Review of H. Satzinger, Die negativen Konstruktionen im Alt- und Mittelägyptischen (Münchner Ägyptologische Studien 12; Berlin, 1968), Journal of Egyptian Archaeology 56, 205-14.

Givón, Talmy

2001 Syntax. An Introduction, 2nd ed. 2 vols. Amsterdam and Philadelphia.

Glanville, Stephen R. K.

1928 "The Letters of Aahmōse of Peniati," Journal of Egyptian Archaeology 14, 294-312.

Goddard, Cliff

2002 "The Search for the Shared Semantic Core of all Languages," in Cliff Goddard and Anna Wierzbicka, ed., Meaning and Universal Grammar - Theory and Empirical Findings (Amsterdam), I, 5-40.

Goedicke, Hans

1955 "The Egyptian Idea of Passing from Life to Death," Orientalia 24, 225-39.

1967 Königliche Dokumente aus dem alten Reich. Ägyptologische Abhandlungen 14. Wiesbaden.

1972 "The Letter to the Dead Naga ed-Deir N 3500," Journal of Egyptian Archaeology 58, 95-98.

1990 “About an Early Use of the Emphatic Possessive Expression," Varia Aegyptiaca 6, 139-60.

Goelet, Ogden, Jr.

1986 "The Term śtp-s? in the Old Kingdom and Its Later Development," Journal of the American Research Center in Egypt 23, 85-98.

Goldwasser, Orly

1999 "Low' and 'High' Dialects in Ramesside Egyptian," in Stefan Grunert and Ingelore Hafemann, ed., Textcorpus und Wörterbuch: Aspekte zur ägyptischen Lexicographie (Probleme der Ägyptologie 14; Leiden), 311-28.

2002 Prophets, Lovers and Giraffes: Wor(l)d Classification in Ancient Egypt. Göttinger Orientforschungen IV, 38. Wiesbaden.

Goyon, Jean-Claude

2012 Le recueil de prophylaxie contre les agressions des animaux venimeux du Musée de Brooklyn Papyrus Wilbour 47.2118.138. Studien zur spätägyptischen Religion 5. Wiesbaden 2012.

\section{Gozzoli, Roberto E.}

2006 The Writing of History in Ancient Egypt during the First Millennium BC (ca. 1070-180 BC): Trends and Perspectives. Golden House Publications: Egyptology 5. London.

Gracia Zamacona, Carlos

2008 Les verbes de mouvement dans les textes des sarcophages: étude sémantique, PhD dissertation, École Pratique des Hautes Études, Paris (unpublished). 
(Gracia Zamacona, Carlos)

2010 "Space, Time, and Abstract Relations in the Coffin Texts." Zeitschrift für Agyptische Sprache und Altertumskunde 137, 13-26.

Grandet, Pierre

1998 Contes de l'Égypte ancienne. Paris.

Grandet, Pierre, and Bernard Mathieu

2003 Cours d'égyptien hiéroglyphique, Paris.

Grapow, Hermann

1914 "Über die Wortbildungen mit einem Präfix $m$ - im Altägyptischen," Abhandlungen der Königlich Preußischen Akademie der Wissenschaften, Philosophisch-historische Klasse 5. Berlin.

Greenberg, Joseph H.

1963 "Some Universals of Grammar with Particular Reference to the Order of Meaningful Elements," in Joseph H. Greenberg, ed., Universals of Language (Cambridge), 73-113.

Griffith, Francis Ll.

1898 The Petrie Papyri: Hieratic Papyri from Kahun and Gurob (Principally of the Middle Kingdom), 2 vols. London.

Grossman, Eitan

to appear "Grammatical Variation and Language Change: the Case of Greek Loan-verbs in Coptic," in Jennifer Cromwell and Eitan Grossman, ed., Beyond Free Variation: Scribal Repertoires in Egypt from the Old Kingdom to the Early Islamic Period, Oxford, 14-16 September 2009.

Grossman, Eitan, and Stéphane Polis

2012 "Navigating Polyfunctionality in the Lexicon: Semantic Maps and Ancient Egyptian Lexical Semantics," in Eitan Grossman et al., ed., Lexical Semantics in Ancient Egyptian (Lingua Aegyptia Studia Monographica 9; Hamburg), 175-225.

Grossman, Eitan, Stéphane Polis, Andréas Stauder, and Jean Winand

2014 ed., On Forms and Functions: Studies in Ancient Egyptian Grammar. Lingua Aegyptia Studia Monographica 15. Hamburg.

Grossman, Eitan, Stéphane Polis, and Jean Winand

2012 ed., Lexical Semantics in Ancient Egyptian. Lingua Aegyptia Studia Monographica, 9. Hamburg.

Grunert, Stefan

2005 "Kulturelle Vergesslichkeit? Zu obsoleten Wörtern aus Texten des Alten Reiches," in Stephan J. Seidelmayer, ed., Texte und Denkmäler des ägyptischen Alten Reiches (Thesaurus Linguae Agyptiae 3; Berlin), 125-42.

2011 'Danse macabre: ein altägyptischer 'Totentanz' aus Saqqara," Studien zur Altägyptischen Kultur 40, 113-36.

Guglielmi, Waltraud

1973 Reden, Rufe und Lieder auf altägyptischen Darstellungen der Landwirtschaft, Viehzucht, des Fisch-und Vogelfangs vom Mittleren Reich bis zur Spätzeit. Tübinger Ägyptologische Beiträge 1. Bonn.

Gundacker, Roman

2011 "On the Etymology of the Egyptian Crown Name mrsw.t: an 'Irregular' Subgroup of $m$-prefix Formations," Lingua Aegyptia 19, 37-86.

Gunn, Battiscombe

1924 Studies in Egyptian Syntax. Paris.

1948 “A Negative Word in Old Egyptian,” Journal of Egyptian Archaeology 34, 27-30.

Gyuris, Beáta

2009 "Quantificational Contrastive Topic with Verum/Falsum Focus," Lingua 119, 625-49. 
Hafemann, Ingelore

2002 "Zum Zusammenspiel von Semantik und Syntax ägyptischer Verben," Lingua Aegyptia 10, 151-210.

2005 "Textsorten und Textfunktion: Gebrauchstexte im monumentalen Diskurs des ägyptischen Alten Reiches," in Stephan J. Seidelmayer, ed., Texte und Denkmäler des ägyptischen Alten Reiches (Thesaurus Linguae Agyptiae 3; Berlin), 143-64.

Hale, Kenneth, and Samuel J. Keyser

1993 "On Argument Structure and the Lexical Expression of Syntactic Relations," in Kenneth Hale and Samuel J. Keyser, ed., The View from Building 20: Essays in Linguistics in Honor of Sylvain Boxberger (Current Studies in Linguistics 24; Cambridge, Massachusetts), 53-109.

Hammamat Jean Couyat and Pierre Montet. Les Inscriptions hiéroglyphiques et hiératiques du Ouâdi Hammâmât. Mémoires de l'Institut Français d'Archéologie Orientale 34. Cairo, 1912.

Hannig, Rainer

1982 “Zum mittelägyptischen Tempussystem," Göttinger Miszellen 56, 35-52.

1986 "Der Kernbereich des mittelägyptischen Verbalsystems, I: Die Infinitivtempora," Journal of Ancient Civilizations, 1, 21-125.

1991 Pseudopartizip und sdm.n=f: Der Kernbereich des mittelägyptischen Verbalsystems 2. Hildesheimer Ägyptologische Beiträge 32. Hildesheim.

1995 Großes Handwörterbuch Ägyptisch-Deutsch, die Sprache der Pharaonen (2800-950 v. Chr.). Kulturgeschichte der Antiken Welt 64. Mainz.

2003 Hannig-Lexica, IV. Ägyptisches Wörterbuch I: Altes Reich und Erste Zwischenzeit. Kulturgeschichte der Antiken Welt 98. Mainz.

2006 Hannig-Lexica, V. Agyptische Wörterbuch II: Mittleres Reich und Zweite Zwischenzeit, 2 vols. Kulturgeschichte der Antiken Welt 112. Mainz.

Hardy, Heather K., and Lynn Gordon

1980 "Types of Adverbial and Modal Constructions in Tolkapaya," International Journal of American Linguistics 46:3, 183-96.

Harley, Heidi, and Elizabeth Ritter

2002 "Person and Number in Pronouns: a Feature-geometric Analysis," Language 78, 482-526.

Haspelmath, Martin

1990 "The Grammaticalization of Passive Morphology," Studies in Language 14, 25-72.

1994 "Passive Participles across Languages," in Barbara Fox and Paul Hopper, ed., Voice: Form and Function (Typological Studies in Language 27; Amsterdam and Philadelphia), 151-77.

1997 Indefinite Pronouns. Oxford Studies in Typology and Linguistic Theory. Oxford.

2003 "The Geometry of Grammatical Meaning: Semantic Maps and Cross-Linguistic Comparison," in Michael Tomasello, ed., The New Psychology of Language (Mahwah, NJ), II, 211-42.

2006 "Against Markedness (and What to Replace It with)," Journal of Linguistics 42:1, 25-70.

Hassan, Selim

1936 Excavations at Gîza 1930-1931. Cairo.

1975 Excavations at Saqqara 1937-1938, ed. by Zaky Iskander. 3 vols. Cairo.

Hatnub Rudolf Anthes, Die Felsinschriften von Hatnub. Untersuchungen zur Geschichte und Altertumskunde Ägyptens 9. Leipzig, 1928.

Haviland, Susan E., and Herbert H. Clark

1974 "What's New? Acquiring New Information as a Process in Comprehension,” Journal of Verbal Learning and Verbal Behavior 13, 512-21. 
Hays, Harold M.

2012 The Organization of the Pyramid Texts, Typology and Disposition, 2 vols. Probleme der Ägyptologie 31. Leiden and Boston.

$H b$. See Werning 2011, II.

Helck, Wolfgang

1972 "Zur Frage der Entstehung der ägyptischen Literatur," Wiener Zeitschrift für die Kunde des Morgenlandes 63/64, 6-26.

1975 Historisch-biographische Texte der 2. Zwischenzeit und neue Texte der 18. Dynastie. Kleine Ägyptische Texte 6. Wiesbaden.

Heqanakht See Allen 2002.

Höhle, Tilman N

1992 "Über Verum-Fokus im Deutschen," in Joachim Jacobs, ed., Informationsstruktur und Grammatik (Linguistische Berichte Sonderhefte 10; Wiesbaden), 112-41.

Hodge, Carleton T.

1954 “An Outline of Middle Egyptian Grammar," Studies in Linguistics 12, 8-23.

Hoekstra, Teun

1988 "Small Clause Results," Lingua 74, 101-39.

Holmberg, Anders, and Ian Roberts

2010 "Introduction: Parameters in Minimalist Theory," in Theresa Biberauer et al., ed., Parametric Variation:

Null Subjects in Minimalist Theory (Cambridge, MA), 1-57.

Hopper, Paul, and Sandra Thompson

1980 "Transitivity in Grammar and Discourse," Language 56, 251-99.

Horn, Laurence R.

2001 A Natural History of Negation. The David Hume Series: Philosophy and Cognitive Science Reissues. Stanford (originally published 1989).

Huang, Yan

2007 Pragmatics. Oxford Textbooks in Linguistics. Oxford.

Ipuwer Roland Enmarch, The Dialogue of Ipuwer and the Lord of All. Griffith Institute Publications. Oxford, 2005.

Israel, Michael

2011 The Grammar of Polarity: Pragmatics, Sensitivity, and the Logic of Scales. Cambridge Studies in Linguistics 127. Cambridge.

Jaeggli, Osvaldo A., and Kenneth J. Safir

1989 "The Null Subject Parameter and Parametric Theory," in Osvaldo A. Jaeggli and Kenneth J. Safir, ed., The Null Subject Parameter (Studies in Natural Language and Linguistic Theory 15; Dordrecht), 1-44.

James, T. G. H.

1962 The Hekanakhte Papers and Other Early Middle Kingdom Documents. Publications of the Metropolitan Museum of Art Egyptian Expedition 19. New York.

Jansen-Winkeln, Karl

1997 “Intensivformen und 'verbale Pluralität' im Ägyptischen,” Lingua Aegyptia 5, 123-36.

2004 "Sprachliche Bemerkungen zu den 'Unterweltsbüchern'," Studien zur Altägyptischen Kultur 32, 205-32.

Jéquier, Gustave

1933 Service des Antiquités de l'Égypte, Fouilles à Saqqarah. Les pyramides des reines Neit et Apouit. Cairo.

1935 Service des Antiquités de l'Égypte, Fouilles à Saqqarah. La pyramide d'Aba. Cairo.

1936 Service des Antiquités de l'Égypte, Fouilles à Saqqarah. Le monument funéraire de Pepi II, vol. I: Le tombeau royal. Cairo. 
Jespersen, Karl

1917 Negation in English and Other Languages (Det Kgl. Danske Videnskabernes Selskab, Historiskfilologiske Meddelelser I, 5). Copenhagen.

Jucker, Andreas H.

2006 "Historical pragmatics," in Keith Brown, ed., Encyclopedia of Language and Linguistics, 2nd ed. (Oxford), 329-32.

Julien, Marit

2002 Syntactic Heads and Word Formation. Oxford Studies in Comparative Syntax. Oxford.

Junge, Friedrich

1978 Syntax der mittelägyptischen Literatursprache: Grundlagen einer Strukturtheorie. Mainz.

1979 "Der Gebrauch von jw im mittelägyptischen Satz," in Manfred Görg und Edgar Pusch, ed., Festschrift Elmar Edel, 12. März 1979 (Ägypten und Altes Testament 1; Bamberg), 263-71.

1985 "Sprache," Lexikon der Ägyptologie 5, 1176-1211.

1989 "Emphasis" and Sentential Meaning in Middle Egyptian. Göttinger Orientforschungen IV, 20. Göttingen.

Junker, Hermann

1940 Gîza IV. Die Mașțaba des k’jmnhh (Kai-em-anch). Österreichische Akademie der Wissenschaften, Denkschriften der Philosophisch-historische Klasse 71, 1. Vienna and Leipzig.

1943 Gîza VI. Die Maștabas des Nfr (Nefer), Kdfjj (Kedfi), K’ḩjf (Kahjef) und die westlich anschließenden Grabanlagen. Österreichische Akademie der Wissenschaften, Philosophisch-historische Klasse, Denkschriften 72,1 . Vienna.

1953 Gîza XI. Der Friedhof südlich der Cheopspyramide, Ostteil. Österreichische Akademie der Wissenschafter, Philosophisch-historische Klasse, Denkschriften 74, 2. Vienna.

Kahl, Jochem, Nicole Kloth, and Ursula Zimmermann

1995 Die Inschriften der 3. Dynastie: eine Bestandsaufnahme. Ägyptologische Abhandlungen 56.Wiesbaden. Kammerzell, Frank

1990 "Funktion und Form: zur Opposition von Perfekt und Pseudopartizip im Alt- und Mittelägyptischen," Göttinger Miszellen 117/119, 181-202.

1991a "Personalpronomina und Personalendungen im Altägyptischen," in Daniela Mendel and Ulrike Claudi, ed., Ägypten im afro-orientalischen Kontext: Aufsätze zur Archäologie, Geschichte und Sprache eines unbegrenzten Raumes, Gedenkschrift Peter Behrens (Afrikanistische Arbeitspapiere, Sondernummer; Köln), 177-203.

1991b "Augment, Stamm und Endung: Zur morphologischen Entwicklung der Stativkonjugation," Lingua Aegyptia 1, 165-99.

1993 "Die altägyptische Negation w: Versuch einer Annäherung," Lingua Aegyptia 3, 17-32.

1998 "Sprachkontakte und Sprachwandel im Alten Ägypten." Habilitation thesis, Georg-August-Universität Göttingen (unpublished).

2005 "Old Egyptian and Pre-Old Egyptian: Tracing linguistic Diversity in Archaic Egypt and the Creation of the Egyptian Language," in Stephan J. Seidelmayer, ed., Texte und Denkmäler des ägyptischen Alten Reiches (Thesaurus Linguae Agyptiae 3; Berlin), 165-229.

Kanawati, Naguib

2007 Deir el-Gebrawi, II: The Southern Cliff. The Australian Centre for Egyptology: Reports 25. Oxford.

Kanawati, Naguib, and Ali Hassan

1996 The Teti Cemetery at Saqqara, I: The Tomb of Nedjet-em-pet, Ka-aper and Others. The Australian Centre for Egyptology: Reports 8. Sydney. 
(Kanawati, Naguib, and Ali Hassan)

1997 The Teti Cemetery at Saqqara, II: The Tomb of Ankhmahor. The Australian Centre for Egyptology: Reports 9. Warminster.

Kanawati, Naguib, and Mohamed A. Abdel Razeq

1999 The Teti Cemetery at Saqqara V: The Tomb of Hesi. The Australian Centre for Egyptology: Reports 13. Warminster.

Kaplony, Peter

1973 "Die altesten Texte," in Serge Sauneron, ed., Textes et langages de l'Égypte pharaonique: cent cinquante années de recherches 1822-1972. Hommages à Jean-François Champollion, 3 vols. (Bibliothèque d'étude 64; Cairo), II, 3-13.

Kay, Paul

1997 "Even," in Paul Kay, Words and the Grammar of Context (CSL Lecture Notes 40. Stanford), 49-98 (originally published 1990).

Kayne, Richard

1994 The Antisymmetry of Syntax. Linguistic Inquiry Monographs 25. Cambridge.

Kheti Wolfgang Helck, Die Lehre des Dw'-Htjj, 2 vols. Kleine Ägyptische Texte 3-4. Wiesbaden, 1970.

Kiss, Katalin É.

1995 "Discourse Configurational Languages: Introduction," in Katalin É. Kiss, ed., Discourse Configurational Languages (Oxford Studies in Comparative Syntax; Oxford), 3-27.

Klebs, Luise

1922 Material zur ägyptischen Kulturgeschichte, II. Die Reliefs und Malereien des mittleren Reiches (VII.XVII. Dynastie ca 2475-1580 v. Chr.). Abhandlungen der Heidelberger Akademie der Wissenschaften, Philosophisch-historische Klasse 3. Heidelberg.

Kloth, Nicole

2002 Die (auto-)biographischen Inschriften des ägyptischen Alten Reiches: Untersuchungen zu Phraseologie und Entwicklung. Hamburg.

Klotz, David

2010 "Two Studies on the Late Period Temples at Abydos," Bulletin de l'Institut Français d'Archéologie Orientale 110, 127-63.

2013 "The Mortuary Texts on Statue JE 38001 (K. 603)," Göttinger Miszellen 236, 43-49.

Knauf, Axel

1986 "Bemerkungen zum ägyptisch-semitischen Sprachvergleich,” Göttinger Miszellen 94, 45-58.

König, Ekkehard

1997 "Zur Bedeutung der Modalpartikeln im Deutschen, ein Neuansatz im Rahmen der Relevanztheorie," Germanistische Linguistik 136, 57-75.

Koopman, Hilda, and Dominique Sportiche

1991 "The Position of Subjects," Lingua 85, 211-58.

Kouwenberg, N. J. C.

2010 The Akkadian Verb and Its Semitic Background. Winona Lake, IN.

Kramer, Ruth

2009 "VSO and SVO Order in Middle Egyptian," in Charles G. Häberl, ed., Afroasiatic Studies in Memory of Robert Hetzron: Proceedings of the Proceedings of the 35th Annual North American Conference on Afroasiatic Linguistics (NACAL 35; Cambridge, MA), 31-75.

Kratzer, Angelika

1996 "Severing the External Argument from Its Verb," in Johan Rooryck and Laurie Zaring, ed., Phrase Structure and the Lexicon (Studies in Natural Language and Linguistic Theory 33; Dordrecht), 109-37. 
2000 "Building Statives," in Lisa J. Conathan et al., ed., Proceedings of the 26th Annual Meeting of the Berkeley Linguistic Society (BLS 26; Berkeley), 385-99.

KRI Kenneth A. Kitchen, Ramesside Inscriptions, Historical and Biographical, 8 vols. Monumenta Hannah Sheen Dedicata 1 and 3-5 (vols. 4-8). Oxford, 1975-90.

Kruchten, Jean-Marie

1982 "Convention et innovation dans un texte royal du début de l'époque ramesside: la stèle de l'an 1 de Séthi ler découverte à Beith-Shan (Musée archéologique de Jérusalem, no. 5.884)," Annuaire de l'Institut de Philologie et d'Histoire Orientales et Slaves 26, 21-62.

1999 "From Middle to Late Egyptian," Lingua Aegyptia 6, 1-97.

Kubisch, Sabine

2008 Lebensbilder der 2. Zwischenzeit. Biographische Inschriften der 13.-17. Dynastie. Sonderschrift des Deutschen Archäologischen Instituts, Kairo 34. Berlin and New York.

Lacau, Pierre, and Henri Chevrier

1956-69 Une chapelle de Sésostris Ier à Karnak, 2 vols. Cairo.

Lambdin, Thomas O.

1958 "The Bivalence of Coptic 'Eta' and Related Problems in the Vocalization of Egyptian," Journal of Near Eastern Studies 17, 177-93.

Lambrecht, Knud

1994 Information Structure and Sentence Form: Topic, Focus, and the Mental Representations of Discourse Referents. Cambridge Studies in Linguistics 71. Cambridge.

Langacker, Ronald W.

1995 "Possession and possessive constructions," in John R. Taylor and Robert E. MacLaury, ed., Language and Cognitive Construal of the World (Trends in Linguistics: Studies and Monographs 82; Oxford), 51-79.

Lapp, Günther

1997 Catalogue of Books of the Dead in the British Museum, I. The Papyrus of Nu (BM EA 10477). London.

2004 Catalogue of the Books of the Dead in the British Museum, III. The Papyrus of Nebseni (BM EA 9900). London.

Larson, Richard K.

1988 "On the Double Object Construction," Linguistic Inquiry 19, 335-91.

Lazard, Gilbert

1998 "De la transitivité restreinte à la transitivité généralisée," in André Rousseau, ed., La transitivité (Paris), 55-84.

LD Richard Lepsius, Denkmaeler aus Aegypten und Aethiopien, 6 vols. Berlin, 1849-59.

Leclant, Jean

1961 Montouemhat: quatrième prophète d'Amon, Prince de la ville. Bibliothèque d'étude 35. Cairo.

1974 "Les textes des pyramides," in Serge Sauneron, ed., Textes et langages de l'Égypte pharaonique: cent cinquante années de recherches 1822-1972. Hommages à Jean-François Champollion, 3 vols. (Bibliothèque d'étude 64; Cairo), II, 37-52.

Leclant, Jean, and Catherine Berger el-Naggar

2001 ed., Les textes de la pyramide de Pépy Ier, 2 vols. Mémoires de l'Institut Français d'Archéologie Orientale 118. Cairo.

Lefebvre, Gustave

1924 Le tombeau de Pétosiris. Cairo.

LES Alan H. Gardiner, Late-Egyptian Stories. Bibliotheca Aegyptiaca 1. Brussels, 1932. 
Levin, Beth, and Malka Rappaport Hovav

1995 Unaccusativity: at the Syntax-Lexical Semantics Interface. Linguistic Inquiry Monograph 26. Cambridge, MA.

Li, Charles N. and Sandra A. Thompson

1976 "Subject and Topic: A New Typology of Language," in Charles N. Li, ed., Subject and Topic (New York), 457-89.

Lichtheim, Miriam

1973 Ancient Egyptian Literature, I: The Old and Middle Kingdoms. Berkeley, Los Angeles and London.

Lipiński, Edward

2001 Semitic Languages: Outline of a Comparative Grammar, 2nd ed. Orientalia Lovaniensa Analecta 80. Leuven.

Longacre, Robert E.

1996 The Grammar of Discourse, 2nd ed. Topics in Language and Linguistics. New York.

López, Jesús

2005 Cuentos y fabulas del Antiguo Egipto. Pliegos de Oriente 9. Mandrid and Barcelona.

Loprieno, Antonio

1980 "Osservazioni sullo sviluppo dell'articolo propositivo in egiziano e nelle lingue semitiche," Oriens Antiquus 19, 1-27.

1984 “Aspekt und Diathese im Ägyptischen,” in F. Junge, ed., Studien zu Sprache und Religion Ägyptens zu Ehren von Wolfhart Westendorf überreicht von seinen Freunden und Schülern. vol. 1: Sprache (Göttingen), 87-102.

1986 Das Verbalsystem im Ägyptischen und im Semitischen: zur Grundlegung einer Aspekttheorie. Göttinger Orientforschungen IV, 17. Wiesbaden.

1991 "Topics in Egyptian Negations," in Daniela Mendel and Ulrike Claudi, ed., Ägypten im AfroOrientalischen Kontext, Aufsätze zur Archäologie, Geschichte und Sprache eines unbegrenzten Raumes: Gedenkschrift Peter Behrens (Afrikanistische Arbeitspapiere; Cologne), 213-35.

1995 Ancient Egyptian. A Linguistic Introduction, Cambridge.

2006 "On fuzzy boundaries in Egyptian syntax," in Gerald Moers et al, ed.,jn.t dr.w: Festschrift für Friedrich Junge (Göttingen), 429-41.

Luft, Ulrich

1992 Das Archiv von Illahun: Briefe I. Hieratische Papyri aus den Staatlichen Museen zu Berlin, Preußischer Kulturbesitz, I. Berlin.

Luraghi, Silvia

2000 "Synkretismus," in Geert E. Booij et al., ed., Morphologie: ein internationales Handbuch zur Flexion und Wortbildung, vol. 1 (Handbücher zur Sprach- und Kommunikationswissenschaft 17:1; Berlin and New York), 638-47.

Lutz, Henry

1927 Egyptian Tomb Steles and Offering Stones of the Museum of Anthropology and Ethnology of the University of California. University of California Publications, Egyptian Archaeology. Leipzig.

Malaise, Michel, and Jean Winand

1999 Grammaire raisonnée de l'égyptien classique. Aegyptiaca Leodiensia 6. Liège.

Manassa, Colleen

2006 "The Crimes of Count Sabni Reconsidered," Zeitschrift für Ägyptische Sprache und Altertumskunde 133, $151-63$. 


\section{Manuelian, Peter Der}

1986 "An Essay in Document Transmission: Nj- $k$ ’’- $n h$ and the earliest hrjw rnpt," Journal of Near Eastern Studies 45, 1-18.

1994 Living in the Past: Studies in Archaism of the Egyptian Twenty-sixth Dynasty. London and New York, Marette, Auguste

1889 Les mastabas de l'Ancien Empire. Fragment du dernier ouvrage d'Auguste Mariette, publié d'après le manuscrit de l'auteur par Gaston Maspero. Paris.

Mathieu, Bernard

2004 "La distinction entre Textes des Pyramides et Textes des Sarcophages est-elle légitime?," in Susanne Bickel and Bernard Mathieu, ed., D'un monde à l'autre: Textes des Pyramides et Textes des Sarcophages, actes de la table ronde internationale "Textes des Pyramides versus Textes des Sarcophages," IFAO 24-26 septembre 2001 (Bibliothèque d'étude 139; Cairo), 247-62.

McCloskey, James

1991 "Clause Structure, Ellipsis and Proper Government in Irish," Lingua 85, 259-302.

McCloskey, James, and Ken Hale

1983 "On the Syntax of Person-Number Inflection in Modern Irish," Natural Language and Linguistic Theory $1,487-534$.

2001 "The Distribution of Subject Properties in Irish," in William D. Davis and Stanley Dubinsky, ed., Objects and Other Subjects: Grammatical Functions, Functional Categories, and Configurationality (Studies in Natural Language and Linguistic Theory 52; Dordrecht), 157-92.

McGregor, William B., and Tamsin Wagner

2006 "The Semantics and Pragmatics of Irrealis Mood in Nyulnyulan Languages," Oceanic Linguistics 4:2, 339-79.

Mehuakhti Elmar Edel, Elmar. "Inschriften des Alten Reiches III: die Stele des Mḥw-3htj (Reisner G 2375)," Mitteilung des Instituts für Orientforschung 1 (1953), 327-33.

Mereruka Sakkarah Expedition. The Mastaba of Mereruka, 2 vols. Oriental Institute Publications 31 and 39. Chicago, 1938.

Merikare Wolfgang Helck. Die Lehre für König Merikare. 2 vols. Kleine Ägyptische Texte 7. Wiesbaden, 1977.

Mink, Gerd

1978 "Allgemeine Sprachwissenschaft und Koptologie," in Robert McL. Wilson, ed., The Future of Coptic Studies (Coptic Studies I; Leiden), 71-103.

Mithun, Marianne

1995 "On the Relativity of Irreality," in Joan L. Bybee and Suzanne Fleischman, ed., Modality in Grammar and Discourse (Typological Studies in Language 32; Amsterdam and Philadelphia), 367-88.

Monaghan, James

1996 "Gardiner, Sir Alan Henderson," in Harro Stammerjohann, ed., Lexicon Grammaticorum: Who's Who in the History of World Linguistics (Tübingen), 325-27.

Montet, Pierre

1936 Les tombeaux dits de Kasr-el-Sayad," Kêmi 6, 81-129.

MOR Eberhard Otto, Das ägyptische Mundöffnungsritual, 2 vols. Ägyptologische Abhandlungen 3. Wiesbaden, 1960 .

Morenz, Ludwig D.

1996 Beiträge zur Schriftlichkeitskultur im Mittleren Reich und in der 2. Zwischenzeit. Ägypten und Altes Testament 29. Wiesbaden.

2004 Bild-Buchstaben und symbolische Zeichen. Orbis Biblicus et Orientalis 205. Fribourg and Göttingen. 
Moret, Alexandre

1931 "La légende d'Osiris à l'époque thébaine d'après l'hymne à Osiris du Louvre," Bulletin de l'Institut Français d'Archéologie Orientale 30, 725-50.

Mostafa, Maha F.

1987 "Kom el-Koffar. Teil II: Datierung und historischen Interpretation des Textes B," Annales du Service des Antiquités de l'Égypte 71, 170-84.

Moussa, Ahmed M., and Hartwig Altenmüller

1977 Das Grab des Nianchchnum und Chnumhotep. Archäologische Veröffentlichungen, Deutsches Archäologisches Institut, Kairo 21. Mainz.

Müller, Dieter

1972 "An Early Egyptian Guide to the Hereafter," Journal of Egyptian Archaeology 58, 99-125.

Müller, Matthias

2012 Review of Borghouts, Egyptian: an Introduction, Lingua Aegyptia 20, 303-10.

Naville, Édouard

1898 The Temple of Deir el Bahari, Part III. Egypt Exploration Fund Memoir 16. London.

Nedjalkov, Vladimir

1988 ed., Typology of Resultative Constructions. Translation ed. by Bernard Comrie. Typological Studies in Language 12. Amsterdam and Philadelphia.

Nedjalkov, Vladimir P., and Sergej J. Jaxontov

1988 "The Typology of Resultative Constructions," in Vladimir P. Nedjalkov, ed., Typology of Resultative Constructions (Typological Studies in Language 12; Amsterdam and Philadelphia), 3-62.

Neferti Wolfgang Helck, Die Prophezeiung des Nfr.tj, 2nd ed. Kleine Ägyptische Texte 10. Wiesbaden, 1992.

Nyord, Rune

2010 "The Radial Structure of Some Middle Egyptian Prepositions," Zeitschrift für Ägyptische Sprache und Altertumskunde 137, 27-44.

Ockinga, Boyo, and Yahia el-Masry

1988 Two Ramesside Tombs at el Mashayikh, I: The Tomb of Anhurmose - The Outer Room. Sydney.

Oréal, Elsa

2005 "Diachronie protoégyptienne et comparaison afroasiatique: la particule $j s$ de déictique à focalisateur," in Alessandro Mengozzi, ed., Studi afroasiatici: IX incontro italiano di linguistica camitosemitica (Bergamo), 69-82.

2007 "Fracture d'actance et dynamique morphosyntaxique: le renouvellement du perfectif en Ancien égyptien," Bulletin de la Société de linguistique de Paris 102, 367-97.

2009 "Same Source, Different Outcomes: A Reassessment of the Parallel between Ancient Egyptian and Akkadian 'Stative' Conjugations," Lingua Aegyptia 17, 183-200.

2010 "Traces of a Stative-Eventive Opposition in Ancient Egyptian: Rethinking Pseudoparticiple as Old Perfective," Zeitschrift für Ägyptische Sprache und Altertumskunde 137, 145-56.

2011 Les particules en égyptien ancien: de l'ancien égyptien à l'égyptien classique. Bibliothèque d'Étude 152. Cairo.

2012 "Discourse Markers between Grammar and Lexicon: Two Ancient Egyptian Case for (De)grammaticialization," in Eitan Grossman et al., ed., Lexical Semantics in Ancient Egyptian (Lingua Aegyptia Studia Monographica 9; Hamburg), 227-45.

Osing, Jürgen

1976a Die Nominalbildung des Ägyptischen, 2 vols. Mainz.

1976b Der spätägyptische Papyrus BM 10808. Ägyptologische Abhandlungen 33. Wiesbaden. 
1987

"Die Partizipien im Ägyptischen und in den Semitischen Sprachen," in Jürgen Osing and Günter Dreyer, ed., Form und Mass: Beiträge zur Literatur, Sprache und Kunst des alten Ägyptens; Festschrift für Gerhard Fecht zum 65. Geburtstag am 6. Februar 1987 (Ägypten und Altes Testatement 12; Wiesbaden), 337-60.

Osing, Jürgen, et al.

1982 Denkmäler der Oase Dachla: aus dem Nachlass von Ahmed Fakhry. Archäologische Veröffentlichungen, Deutsches Archäologisches Institut, Abteilung Kairo 28. Mainz.

Palmer, Frank R.

2000 Mood and Modality, 2nd ed. Cambridge Textbooks in Linguistics. Cambridge.

Pantalacci, Laure

1998 "La documentation épistolaire du palais des gouverneurs à Balat-'Ayn Asil," Bulletin de l'Institut Français d'Archéologie Orientale 98, 303-15.

Parkinson, Richard B.

1991 Voices from Ancient Egypt. An Anthology of Middle Kingdom Writings. London.

1997 The Tale of Sinuhe and Other Ancient Egyptian Poems 1940-1640 BC. Oxford.

2002 Poetry and Culture in Middle Kingdom Egypt: a Dark Side to Perfection. London and New York.

Peas. $\quad$ Richard B. Parkinson, The Tale of the Eloquent Peasant. Oxford, 1991.

pEbers Hermann Grapow, Grundriss der Medizin der Alten Ägypter, V. Die medizinische Texte in hieroglyphischer Umschreibung autographiert. Berlin, 1958.

Perrin, Loïc-Michel

2010 "Polysemous Qualities and Universal Networks, Invariance and Diversity," Linguistic Discovery 8/1, 259-80.

Peters, Stanley, and Dag Westerståhl

2006 Quantifiers in Language and Logic. Oxford.

Petosiris Gustave Lefebvre, Le tombeau de Pétosiris. Cairo, 1924.

Petrie, William M. F.

1900 Dendereh 1898. Egypt Exploration Fund Memoir 17. London.

Peust, Carsten

1999 Egyptian Phonology, an Introduction to the Phonology of a Dead Language. Monographien zur ägyptischen Sprache 2. Göttingen.

2008 "Adjektiv und Adjektivverb im Mittelägyptischen," in Carsten Peust, ed., Miscellanea in Honorem Wolfhart Westendorf(Göttinger Miszellen, Beihefte 3; Göttingen), 58-82.

Pinker, Stephen

1989 Learnability and Cognition: the Acquisition of Argument Structure. Cambridge, MA.

Polis, Stéphane

2009 "Interaction entre modalité et subjectivité en néo-égyptien: autour de la construction $m r i+j w$ circ. 'souhaiter que'," in Matthias Müller and Sami Uljas, ed., Proceedings of the Fourth International Conference on Egyptian Grammar (Crossroads IV): Basel, March 19-22, 2009 (Lingua Aegyptia 17; Göttingen), 201-19.

Pollock, Jean-Yves

1989 "Verb Movement, Universal Grammar, and the Structure of IP," Linguistic Inquiry, 20, 365-424.

Polotsky, Hans J.

1929 Zu den Inschriften der 11. Dynastie. Untersuchungen zur Geschichte und Altertumskunde Ägyptens 11. Leipzig.

1944 Études de syntaxe copte, Cairo.

1957 "The Emphatic $s$ dm.n=fForm," Revue d'Égyptologie 11, 109-17. 
(Polotsky, Hans J.)

1965 Egyptian Tenses. The Israel Academy of Sciences and Humanities, Proceedings II 5. Jerusalem.

1976 "Les transpositions du verbe en égyptien classique," Israel Oriental Studies 6, 1-50.

Posener, Georges

1978 Catalogue des ostraca hiératiques littéraires de Deir el-Médineh, III. Documents de Fouilles 20. Cairo. Posener-Kriéger, Paule, and Jean-Louis de Cénival

1968 Hieratic Papyri in the British Museum, V. The Abu Sir Papyri. London.

Pressl, Diana A.

1993 "Zur Königsideologie der 26. Dynastie: Untersuchungen anhand der Phraseologie der Königsinschriften," Studien zur Altägyptischen Kultur 20, 223-54.

Pries, Andreas $\mathrm{H}$.

2011 Die Stundenwachen im Osiriskult, eine Studie zur Tradition und späten Rezeption von Ritualen im Alten Ägypten. Studien zur spätägyptischen Religion 2. Wiesbaden.

pSmith Hermann Grapow, Grundriss der Medizin der Alten Ägypter, V. Die medizinische Texte in hieroglyphischer Umschreibung autographiert. Berlin, 1958.

PT see Pyr.

Ptahhotep Zbyněk Žába, Les maximes de Ptahhotep. Prague, 1956.

pWestcar Aylward M. Blackman, The Story of King Kheops and the Magicians Transcribed from Papyrus Westcar (Berlin Papyrus 3033), ed. by W.V. Davies. Reading, 1988.

Pyr. $\quad$ Kurt Sethe, Die altägyptischen Pyramidentexte nach den Papierabdrücken und Photographien des Berliner Museums, 4 vols. Leipzig, 1908-22.

Quack, Joachim F.

1996 “' $w$ 'Größe' und ‘ 'Zustand, Art': zwei verwechselbare demotische Wörter," Enchoria 23, 62-75.

2003 “Zum Charakter der 'zweiradikaligen’ Verben des Ägyptischen,” in M. Lionel Bender et al., ed., Selected Comparative-Historical Afrasian Linguistic Studies in Memory of Igor M. Diakonoff (LINCOM Studies in Afroasiatic Linguistics 14; Munich), 167-74.

2013 "Irrungen, Wirrungen? Forscherische Ansätze zur Datierung der älteren ägyptischen Literatur." in Gerald Moers et al., ed., Dating Egyptian Literary Texts. "Dating Egyptian Literary Texts, ” Göttingen, 9-12 June 2010, vol. I (Lingua Aegyptia, Studia Monographica 11; Hamburg), 405-69.

Quibell, James Edward, et al.

1898 The Ramesseum and the Tomb of Ptah-hetep. Egyptian Research Account 2. London.

Reiner, Erica

1966 A Linguistic Analysis of Akkadian. Janua Linguarum Series Practica 21. London, The Hague, and Paris. Reinhart, Tanya

1981 "Pragmatics and Linguistics: an Analysis of Sentence Topics," Philosophica 27, 53-94.

Reintges, Chris H.

1993 "A Functional Reexamination of Hammamat Inscription 191:6," in Silvio Curto et al, ed., Atti di Sesto Congresso Internazionale di Egittologia (2 vols.; Turin), II, 437-45.

1994 "Egyptian Root-and-Pattern Morphology," Lingua Aegyptia 4, 213-44.

1995 "Verbal tenses in Older Egyptian: a Reichenbachian Approach," Göttinger Miszellen 149, 83-97.

1996 "The $s \underline{d} m-t w=f$ as a Morphological Passive," Göttinger Miszellen 153, 79-99.

1997 Passive Voice in Older Egyptian: a Morpho-Syntactic Study. Holland Institute of Linguistics Dissertations 28. Den Haag.

1998 "Mapping Information Structure to Syntactic Structure: One Syntax for jn," Revue d'Égyptologie 49, 195-220. 
2000 "The Licensing of Gaps and Resumptive Pronouns in Older Egyptian Relatives," in Jacqueline Lecarme et al., ed., Research in Afroasiatic Grammar: Papers from the Third Conference on Afroasiatic Languages, Sophia Antipolis, France 1996 (Current Issues in Linguistic Theory 202; Amsterdam and Philadelphia), 243-62.

2004a Coptic Egyptian (Sahidic Dialect): A Learner's Grammar. (Africanist Studybooks 15). Cologne.

$2004 b \quad$ "The Older Egyptian śdm(.w) $=f$ Passive Revisited," Folia Orientalia 40, 51-70.

2005a "The Correlation between Word Order Alternations, Grammatical Agreement and Event Semantics in Older Egyptian," in Katalin É. Kiss, ed., Universal Grammar in the Reconstruction of Ancient Languages (Studies in Generative Grammar 83; Berlin), 31-103.

2005b "On Passive Participles and Relative Forms," Lingua Aegyptia 13, 103-39.

2006 "The Older Egyptian Stative Revisited," Lingua Aegyptia 14, 115-34.

2009 "Spontaneous Syntactic Change," in Paola Crisma and Giuseppe Longobardi, ed., Historical Syntax and Linguistic Theory (Oxford), 41-57.

2011a "The Evolution of the Ancient Egyptian Stative: Diachronic Stability besides Inflectional Change," Folia Orientalia 48, 7-97.

2011 b "The Oral-Compositional Form of Pyramid Text Discourse," in Fredrik N. Hagen et al., ed., Narratives of Egypt and the Ancient Near East: Literary and Linguistic Approaches (Orientalia Lovaniensa Analecta 189; Louvain), 3-54.

2012 "Macroparametric Change and the Synthetic-Analytic Continuum: The Case of Ancient Egyptian," in Charlotte Galves et al., ed., Parameter Theory and Linguistic Change (Oxford Studies in Diachronic and Historical Linguistics 2; Oxford), 144-69.

2013 "Sapirian 'Drift' towards Analyticity and Long-term Morphosyntactic Change in Ancient Egyptian," in Ritsuko Kikusawa and Laurence A. Reid, ed., Historical Linguistics 2011: Selected Papers from the 20th International Conference on Historical Linguistics, Osaka, 25-30 July 2011 (Current Issues in Linguistic Theory 326; Amsterdam and Philadelphia), 289-328.

2015 "The Old and Early Middle Egyptian Stative: Morphosyntax • Semantics • Typology," in Eitan Grossman et al., ed., Egyptian-Coptic Linguistics in Typological Perspective (Empirical Approaches to Language Typology 55; Berlin), 387-454.

Ritter, Elisabeth

1995 "On the Syntactic Category of Pronouns and Agreement," Natural Language and Linguistic Theory 13, $405-43$.

Ritter, Thomas

1995 Das Verbalsystem der königlichen und privaten Inschriften XVIII. Dynastie bis einschließlich Amenophis III. Göttinger Orientforschungen IV, 30. Wiesbaden.

Rizzi, Luigi

1982 Issues in Italian Syntax. Studies in Generative Grammar 11. Dordrecht.

1986 "Null Objects in Italian and the Theory of Pro," Linguistic Inquiry 17, 501-57.

1997 "The Fine Structure of the Left Periphery," in Liliane Haegeman, ed., Elements of Grammar: Handbook of Generative Syntax (Kluwer International Handbooks of Linguistics 1; Dordrecht), 281-337.

Roberts, Ian G.

1993 Verbs and Diachronic Syntax: A Comparative History of English and French. Studies in Natural Languages and Linguistic Theory 28. Dordrecht.

Rothe, Russell D., William K. Miller, and George Rapp

2008 Pharaonic Inscriptions from the Southeastern Desert of Egypt. Winona Lake, IN. 
Russo, Barbara

2102 "Funerary Spells at Saqqara South: Some Considerations about the Inscriptions of Anu's Coffin (Sq20X) and Their Date," Zeitschrift für Ägyptische Sprache und Altertumskunde 139, 80-92.

Sandman, Maj

1938 Texts from the Time of Akhenaten. Bibliotheca Aegyptiaca 8. Brussels.

Satzinger, Helmut

1986 "Syntax der Präpositionsadjective ('Präpositionsnisben')," Zeitschrift für Ägyptische Sprache und Altertumskunde 113, 141-53.

1987 "On Tense and Aspect in Middle Egyptian," in Gertie Englund and Paul J. Frandsen, ed., Crossroad: Chaos or the Beginning of a New Paradigm. Papers from the Conference on Egyptian grammar, Helsingør 28-30 May 1986 (Copenhagen), 297-313.

1988 "Towards Reconstructing the Tense System of Old Egyptian," in John D. Ray, ed., Lingua sapientissima: a Seminar in Honour of H. J. Polotsky Organised by the Fitzwilliam Museum, Cambridge and the Faculty of Oriental Studies in 1984 (Cambridge), 72-79.

1993a “Emphase' oder die Rhematisierung eines adverbialen Komplements im Ägyptischen,” Wiener Zeitschrift für die Kunde des Morgenlandes 83, 189-206.

1993b "Die Protasis jr s d dm.fim älteren Ägyptisch," Lingua Aegyptia 3, 121-35.

1999a "Koptische Vokalphoneme und ägyptische Pluralformation," in Stephen Emmel, ed., Agypten und Nubien in spätantiker und christlicher Zeit: Akten des 6. Internationalen Koptologenkongresses Münster 20. - 26. Juli 1996 (Sprachen und Kulturen des christlichen Orients 6; Wiesbaden), II, 365-74.

1999b "Observations in the Field of the Afroasiatic Suffix Conjugation," in Marcello Lamberti and Livia Tonelli, ed., Afroasiatica Tergestina: Papers from the 9th Italian Meeting of Afro-Asiatic (Hamito-Semitic) Linguistics, Trieste, April 23-24, 1998 (Contributi presentati al $9^{\circ}$ Incontro di linguistica afroasiatica (camito-semitica), Trieste, 23-24 aprile 1998; Padova), 23-33.

2007 "Anmerkungen zu semitischen Suffixkonjugation," in Rainer M. Voigt, ed., "From beyond the Mediterranean:" Akten des 7. Internationalen Semitohamitistenkongresses (VII. ISHaK), Berlin 13. bis 15. September 2004 (Aachen), 493-500.

2012 "On Egyptian Participles and Nomina Agentis." Folia Orientalia 49, 467-76.

2013 Review of Wolfgang Schenkel, Tübinger Einführung in die klassisch-ägyptische Sprache und Schrift (2012), Lingua Aegyptia 21, 303-309.

to appear "Semitic Suffix Conjugation and Egyptian Stative: A Hypothetic Morpho-Syntactic Scenario of its Origin" (manuscript available at http://homepage.univie.ac.at/helmut.satzinger/ Texte/Stative_Sem.pdf; accessed: 20 Jan. 2013).

Säve-Söderbergh, Torgny

1994 The Old Kingdom Cemetery at Hamra Dom (el-Qasr wa es-Saiyad). Stockholm.

Scharff, Alexander

1924 "Briefe aus Illahun," Zeitschrift für Ägyptische Sprache und Altertumskunde 59, 20-51.

Schenkel, Wolfgang

1965a “'Singularisches' und 'pluralisches' Partizip," Mitteilungen des Deutschen Archäologischen Instituts, Abteilung Kairo 20, 110-14.

1965b Memphis - Herakleopolis - Theben: die epigraphische Zeugnisse der 7.-11. Dynastie Ägyptens. Ägyptologische Abhandlungen 12. Wiesbaden.

1965c "Beiträge zur mittelägyptischen Syntax III: Sätze mit Pseudopartizip," Zeitschrift für Ägyptische Sprache und Altertumskunde 92, 47-68.

1968 "Syntagmen mit infiniten Verbalformen als Transformate von Sätzen," Mitteilungen des Deutschen Archäologischen Instituts, Abteilung Kairo 23, 167-81. 
1971

1981

$1983 a$

1983b

1985

1990

$1994 a$

$1994 b$

1996

1998

1999

$2000 \mathrm{a}$

$2000 \mathrm{~b}$

2002

2004

$2005 \mathrm{a}$

$2005 b$

$2005 \mathrm{c}$

2006

$2007 \mathrm{a}$

$2007 b$

$2008 \mathrm{a}$

$2008 \mathrm{~b}$

"Das altägyptische Pseudopartizip und das indogermanische Medium/Perfekt," Orientalia 40, 301-16. "sd $\underline{d} m=f$ und $s \underline{d} . w . w=f$ als Prospektivformen," in Dwight W. Young, ed., Studies Presented to Hans Jakob Polotsky. (East Gloucester, MA), 506-17.

Aus der Arbeit an einer Konkordanz zu den altägyptischen Sargtexten. Göttinger Orientforschungen IV, 12. Wiesbaden.

Zur Rekonstruktion der deverbalen Nominalbildung des Ägyptischen. Göttinger Orientforschungen IV, 13. Wiesbaden.

"Zur Verbalflexion der Pyramidentexte," Bibliotheca Orientalis 42, 481-94.

Einführung in die altägyptische Sprachwissenschaft. Darmstadt.

"śḉm.t-Perfekt und śç̌m.ti-Stativ: die beiden Pseudopartizipien des Ägyptischen nach dem Zeugnis der Sargtexte," in Heike Behlmer, ed., ... quaerentes Scientiam: Festgabe für Wolfhart Westendorf zu seinem 70. Geburtstag, überreicht von seinem Schülern (Göttingen), 157-82.

"Zur Formenbilding des Verbs im Neuägyptischen," Orientalia 63, 10-15.

"Eine Konkordanz zu den Sargtexten und die Graphien der 1. Person Singular," in Harco Willems, ed., The World of the Coffin Texts (Egyptologische Uitgaven 9; Leuven), 115-27.

Tübinger Einführung in die klassisch-ägyptische Sprache und Schrift. Tübingen.

"Standardtheorie und invertierte Standardtheorie." Zeitschrift für Ägyptische Sprache und Altertumskunde $125,140-60$.

"ś-Kausativa, t-Kausativa und 'innere' Kausativa: die śs-Kausativa der Verben I.ś in den Sargtexten," Studien zur altägyptischen Kultur 27, 313-52.

"Die Endungen des Prospektivs und des Subjunktivs (śḉm=f, śḉm.w=f, śçm.y=f) nach Befunden der Sargtexte," Lingua Aegyptia 7, 27-112.

"Die Endungen des Negativkomplements im Spiegel der Befund der Sargtexte," Lingua Aegyptia 7, 1-27. "Zur Formenbildung des prädikativen sç $m=f$ der Verben II.gem., vornehmlich nach dem Zeugnis der Sargtexte," Göttinger Miszellen 189, 89-98.

"Das śç̆m(.w)=f-Passiv, Perfekt vs. Futur, nach dem Zeugnis der Sargtexte, 1. Teil," Zeitschrift für Ägyptische Sprache und Altertumskunde 131, 173-88.

"Das śḉm $(. w)=f$-Passiv, Perfekt vs. Futur, nach dem Zeugnis der Sargtexte, 2. Teil," Zeitschrift für Ägyptische Sprache und Altertumskunde 132, 40-54.

"'(Sich) fernhalten' und dergleichen in den Sargtexte," in Heinz Felber, ed., Feinde und Aufrüher Konzept von Gegnerschaft in ägyptischen Texten besonders des Mittleren Reiches (Abhandlungen der Sächsischen Akademie der Wissenschaften zu Leipzig. Philologisch-historische Klasse 78, 5; Leipzig), 111-41.

\section{Tübinger Einführung in die klassisch-ägyptische Sprache und Schrift, 4th ed. Tübingen.}

"Von der Morphologie zur Syntax und zurück," Lingua Aegyptia 14, 55-82.

"Die Partikel iw und die Intuition des Interpreten: Randbemerkungen zu Antonio Loprieno, 'On fuzzy boundaries in Egyptian syntax'," Lingua Aegyptia 15, 161-201.

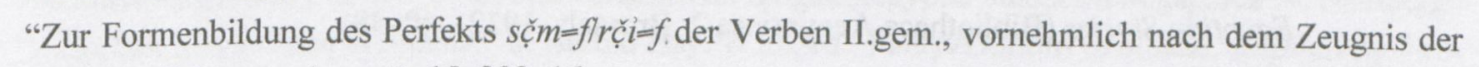
Sargtexte," Lingua Aegyptia 15, 203-16.

"Substantiv / Selbständiges Personalpronomen + enklitisches Personalpronomen, eine grammatische Konstruktion des älteren Ägyptisch?," Göttinger Miszellen 217, 97-109.

"Die ägyptische Nominalbildungslehre und die Realität der hieroglyphischen Graphien der Sargtexte, II: Weitere Nominalbildungsklassen mit einer Endung -w/y/i (A II 5-10, A III 4-6 und A I 7/8/10)," Lingua Aegyptia 16, 153-70. 
(Schenkel, Wolfgang)

2009a "Predikativisches und abstrakt-relativisches sçm. $n=f$ : Beobachtungen an den Verben II.gem und ult. $n$ im Korpus der Sargtexte," in Gideon Goldenberg and Ariel Shisha-Halevy, ed., Egyptian, Semitic and General Grammar: Studies in Memory of H. J. Polotsky (Jerusalem), 40-60.

2009 b "Zur Silbenstruktur des Ägyptischen," Lingua Aegyptia 17, 259-76.

2010 "Die Clèresche Relativform,” Zeitschrift für Ägyptische Sprache und Altertumskunde 137, 66-90.

2011 "Merkmalloses versus pluralisches/distributives/intensives Partizip: Kritik der Ausgangsbeobachtungen," Zeitschrift für Ägyptische Sprache und Altertumskunde 138, 63-78.

2012a Tübinger Einführung in die klassisch-ägyptische Sprache und Schrift, 5th ed. Tübingen.

2012b "Mittelägyptische Grammatik: Von den Texten zu den Texten," Bibliotheca Orientalis 69, 13-42.

2012c " $m=k+$ ini.tw=f?," Göttinger Miszellen 234, 11-13.

2014 "Syntax und Sinnzusammenhang: emphatische Konstruktion, Rang-V-Erweiterung und anderes mehr," in Harold Hays et al., ed., Interpretations of Sinuhe, Inspired by Two Passages (Proceedings of a Workshop Held at Leiden University, 27-29 November 2009) (Egyptologische Uitgaven 27; Leuven), 81-172.

im Druck "Die Farben aus der Sicht der Alten Ägypter."

Schneider, Thomas

2007 "Contextualising the Tale of the Herdsman," in Thomas Schneider and Kasia Szpakowska, ed., Egyptian Stories: a British Egyptological Tribute to A. B. Lloyd at the Occasion of his Retirement (Alter Orient und Altes Testament 347; Münster), 309-18.

Schultheiß, Alexander

2014 "Studien zur Semantik und Formenbildung der Nisbe im Älteren Ägyptischen." PhD dissertation, Philosophische Fakultät III, Humboldt-Universität zu Berlin (unpublished).

Schweitzer, Simon D.

2000 "Untersuchungen zum Wortschatz des Papyrus Westcar und Erstellung einer Liste noch im koptischen belegter Wortformen.” Magister Artium thesis, Universität Münster (unpublished).

2005 Schrift und Sprache der 4. Dynastie. MENES 3. Wiesbaden.

Sethe, Kurt

1899 Das aegyptische Verbum im Altaegyptischen, Neuaegyptischen und Koptischen, 2 vols. Leipzig.

1923 "Die Vokalisation des Ägyptischen," Zeitschrift der Deutschen Morgenländischen Gesellschaft 77.2, 145-207.

1924 "Eine bisher unbekannte enklitische Negation im Altägyptischen," Zeitschrift für Ägyptische Sprache und Altertumskunde 59, 63-64.

1928a Dramatische Texte zualtaegyptischen Mysterienspielen. Untersuchungen zur Geschichte und Altertumskunde Ägyptens 10. Leipzig.

1928b Ägyptische Lesestücke zum Gebrauch im akademischen Unterricht. 2nd ed. Leipzig.

1936-62 Übersetzung und Kommentar zu den altägyptischen Pyramidentexten, 6 vols. Hamburg.

Sh.S.

Aylward M. Blackman, "The Story of the Shipwrecked Sailor," in Aylward M. Blackman, ed., Middle Egyptian Stories (Bibliotheca Aegyptiaca 2. Brussels, 1932), 40-48.

Shisha-Halevy, Ariel

1986 “(i)rf in the Coffin Texts: a Functional Tableau," Journal of the American Oriental Society 106, 641-58. Shlonsky, Ur

1997 Clause Structure and Word Order in Hebrew and Arabic: an Essay in Comparative Semitic Syntax. Oxford Studies in Comparative Syntax 11. Oxford.

Siewierska, Anna

2004 Person. Cambridge Textbook in Linguistics. Cambridge. 
Silverman, David P.

1980 Interrogative Constructions with jn and jn-jw in Old and Middle Egyptian. Bibliotheca Aegyptiaca 1. Malibu.

1981 "Plural Demonstrative Constructions in Ancient Egyptian," Revue d'Égyptologie 33, 59-65.

1985 "Verbal Nominal Clauses in Middle Egyptian," in Paule Posener-Kriéger, ed., Mélanges Gamal Eddin Mokhtar, 2 vols. (Bibliothèque d'étude 47; Cairo), II, 269-85.

2000 "The Threat-Formula and Biographical Texts in the Tomb of Hezi at Saqqara," Journal of the American Research Center in Egypt 37, 1-13.

Simpson, William K.

1966 "The Letter to the Dead from the Tom of Meru (N 3737) at Nag' ed-Deir," Journal of Egyptian Archaeology 52 (1966), 39-50.

1970 "A Late Old Kingdom Letter to the Dead from Nag“ ed-Deir N 3500," Journal of Egyptian Archaeology $56,58-64$.

1974 The Terrace of the Great God at Abydos: the Offering Chapels of Dynasties 12 and 13. Publications of the Pennsylvania-Yale Expedition to Egypt 5. New Haven and Philadelphia.

1980 Giza Mastabas, IV. Mastabas of the Western Cemetery, Part I: Sehemka (G 1029); Tjetu I (G 2001); Iasen (G 2196); Penmeru (G 2197); Hagy, Nefertjentet, and Herunefer (G 2352/53); Djaty, Tjetu II, and Nimesti ( $G$ 2337X, 2343, 2366). Boston.

Sin. $\quad$ Roland Koch, Die Erzählung des Sinuhe. Bibliotheca Aegyptiaca 17. Brussels, 1990.

Sinai Alan H. Gardiner and T. Eric Peet, The Inscriptions of Sinai, ed. by Jaroslav Černý, 2 vols. 2nd ed. Egypt Exploration Society 45. London, 1955.

Siut $\quad$ Francis Ll. Griffith, The Inscriptions of Siût and Dêr Rîfeh. London, 1889.

Smith, Carlotta S.

1997 The Parameter of Aspect. Studies in Linguistics and Philosophy 43. Dordrecht.

Smither, Paul C.

1942 "An Old Kingdom Letter Concerning the Crimes of Count Sabni," Journal of Egyptian Archaeology 28, 16-19.

1945 "The Semnah Dispatches," Journal of Egyptian Archaeology 31, 3-10.

Spencer, Patricia

1984 The Egyptian Temple: a Lexicographical Study. London.

Sperveslage, Gunnar

2010 "Das Kongruenzverhalten von Adjektiven im Altägyptischen," Lingua Aegyptia 18, $217-51$.

Spiegelberg, Wilhelm

1909 "Egyptian Texts," in James E. Quibell, ed., Excavations at Saqqara (1907-1908) (2 vols., Service des Antiquités de l'Égypte 5; Cairo), I, 79-83.

Stauder, Andréas

2010 "Earlier Egyptian Verbal Morphology," unpublished MS.

2013 Linguistic Dating of Middle Egyptian Literary Texts. Lingua Aegyptia Studia Monographica 12. Hamburg.

2014a The Earlier Egyptian Passive: Voice and Perspective. Lingua Aegyptia Studia Monogaphica 14. Hamburg.

2014b "Linguistic Dissonance in Simuhe," in Harold Hays et al., ed., Interpretations of Sinuhe, Inspired by Two Passages (Proceedings of a Workshop Held at Leiden University, 27-29 November 2009) (Egyptologische Uitgaven 27; Leuven), 173-88.

2014c "Splitting the $s \underline{d} m . n=f$ ? A discussion of written forms in Coffin Texts, Part 1," Zeitschrift für Ägyptische Sprache und Altertumskunde 141, 83-96.

2014d "Splitting the $s \underline{d} m . n=f$ ? A discussion of written forms in Coffin Texts, Part 2," Zeitschrift für Ägyptische Sprache und Altertumskunde 141, 196-209. 
(Stauder, Andréas)

2014e "Interpreting written morphology: the $s \underline{d m} . n=f$ in the Pyramid Texts," Journal of Near Eastern Studies $73,253-71$.

2014f "L'émulation du passé à l'ère thoutmoside: la dimension linguistique," in Susanne Bickel, ed., Vergangenheit und Zukunft: Studien zum historischen Bewusstsein in der Thutmosidenzeit (Aegyptiaca Helvetica 22; Basel), 77-125.

Stauder-Porchet, Julie

2008 "Une formule de la biographie éthique: traduction et commentaire," Revue d'Égyptologie 58, 369-80.

2009 La préposition en égyptien de la première phase: approche sémantique. Aegyptiaca Helvetica 21. Basel.

2011 "Les autobiographies événementielles de la Ve dynastie: premier ensemble de textes continus en Égypte," in Miroslav Bárta et al., ed., Abusir and Saqqara in the Year 2010 (2 vols.; Prague), II, 747-66.

to appear Les autobiographies de l'Ancien Empire égyptien: étude sur la naissance d'un genre.

Stowell, Tim

1993 "Subject across Categories," The Linguistic Review 2, 285-312.

Strudwick, Nigel

2005 Texts from the Pyramid Age. Writings from the Ancient World 16. Atlanta.

Sybesma, Rint P. E.

2007 "Whether We Tense-agree Overtly or Not," Linguistic Inquiry 38, 580-87.

Tacke, Nikolaus

2013 Das Opferritual des ägyptischen Neuen Reiches, 2 vols. Orientalia Lovaniensia Analecta 222. Leuven, Paris and Walpole.

Tenny, Carol

1994 Aspectual Roles and the Syntax-Semantics Interface. Dordrecht.

Thacker, Thomas W.

1954 The Relationship of the Semitic and Egyptian Verbal Systems. Oxford.

Thompson, Sandra A., Robert E. Longacre, and Shin Hwang

2007 "Adverbial clauses," in Timothy Shopen, ed., Language Typology and Syntactic Description, vol. II: Complex Constructions (Cambridge), 237-300.

TLA Thesaurus Linguae Aegyptiae and Das digitalisierte Zettelarchiv (DZA), ed. by Berlin-Brandenburgische Akademie der Wissenschaften (Berlin): http://aaew2.bbaw.de/tla/.

Toivonen, Ida

2000 "The Morphosyntax of Finish Possessives," Natural Language and Linguistic Theory 18, 579-609.

TPPI Jacques J. Clère and Jacques Vandier, Textes de la Première Période Intermédiaire et de la Xlème dynastie. Bibliotheca Aegyptiaca 10. Brussels, 1948.

Uljas, Sami

2004 "Cairo Bowl Lines 7-8," Göttinger Miszellen, 201, 95-104.

2007a The Modal System of Earlier Egyptian Complement Clauses: a Study in Pragmatics in a Dead Language. Probleme der Ägyptologie 26. Leiden and Boston.

2007b "The Earlier Egyptian Adjective Reconsidered," Lingua Aegyptia 15, 231-50.

2008 "Methodological Remarks on Defining 'Verb Forms' in Earlier Egyptian, with Specific Reference to the $s \underline{d}=f$ Formation," Lingua Aegyptia 16, 197-212.

2010 "Formally speaking. Observations on a Recent Theory of the Earlier Egyptian sdm.n=f," Lingua Aegyptia $18,253-61$.

2011 "Syncretism and the Earlier Egyptian $s \underset{d}{d m}=f$ : Speculations on Morphological Interconnections across Paradigms," Lingua Aegyptia 19, 155-74. 
2013 "Some Remarks on a Negated Earlier Egyptian Nominal Sentence and Related Constructions," Journal of Egyptian Archaeology 99, 241-51.

Urk.

I Kurt Sethe, Urkunden des ägyptischen Altertums, I. Urkunden des Alten Reichs. 2nd ed. Leipzig, 1933.

IV Kurt Sethe and Wolfgang Helck, Urkunden des ägyptischen Altertums, IV. Urkunden der XVIII. Dynastie. Leipzig and Berlin, 1906-1958.

VII Kurt Sethe, Urkunden des ägyptischen Altertums, VII. Historisch-biographische Urkunden des Mittleren Reiches, ed. by W. Erichsen. Leipzig, 1933.

van den Boorn, G. P. F.

1982 "On the Date of 'The Duties of the Vizier'," Orientalia 51, 369-81.

Vandekerckhove, Hans, and Renate Müller-Wollermann

2001 Elkab VI: Die Felsinschriften des Wadi Hilâl, 2 vols. Brussels.

Vandier, Jacques

1950 Mo'alla: la tombe d'Ankhtifi et la tomb de Sébekhotep. Bibliothèque d'étude 18. Cairo.

van Valin, Robert D., Jr.

2006 Exploring the Syntax-Semantic Interface. Cambridge.

Varille, Alexandre

1968 Inscriptions concernant l'architecte Amenhotep fils de Hapou. Bibliothèque d'étude 44. Cairo.

Vercoutter, Jean

1958 "Une épitaphe royale inédite du Sérapéum (contribution à l'histoire des Apis et Sérapéum de Memphis)," Mitteilungen des Deutschen Archäologischen Instituts, Abteilung Kairo 16, 333-45.

Vergote, Jozef

1969 "The Plural of Nouns in Egyptian and Coptic," Orientalia 38, 77-96.

Verhagen, Arie

2005 Constructions of Intersubjectivity. Discourse, Syntax, and Cognition. Oxford.

Vernus, Pascal

1978 "Littérature et autobiographie: les inscriptions de $s$ 3’-mwt surnommé Kyky," Revue d'Égyptologie 30, 115-46.

1981 “Formes 'emphatiques' en fonction non 'emphatique' dans la protase d'un système corrélatif," Göttinger Miszellen 43, 73-88.

1984a "Sujet + sdm.fet sujet + pseudoparticipe avec les verbes de qualité: dialectique de l'aspect et de l'Aktionsart," in Friedrich Junge, ed., Studien zu Sprache und Religion Ägyptens zu Ehren von Wolfhart Westendorf, überreicht von seinen Freunden und Schülern (2 vols.; Göttingen), I, 197-212.

1984b "Le sdm.n.f des verbes de qualité: dialectique de l'aspect et de l'Aktionsart," Revue d'Égyptologie 35, 171-84.

1985 "Etudes de philologie et de linguistique IV: non-existence et définition du sujet: $b n$ prédicatif en néoégyptien," Revue d'Égyptologie 36, 153-68.

1986a "Aspect and Morphosyntactic Patterns in Middle Egyptian," in Gertie Englund and Paul Frandsen, ed., Crossroad: Chaos or the Beginning of a New Paradigm. Papers from the Conference on Egyptian Grammar, Helsingør 28-30 May 1986 (Carsten Niehbur Institute Publications 1; Copenhagen), 375-88.

1986b "Études de Philologie et de linguistique (V)," Revue d'Égyptologie 37, 141-44.

1988 “L'Egypto-copte," in Jean Perrot, ed., Les langues dans le monde ancien et moderne, troisième partie: Les langues chamito-sémitiques (Paris), 161-206.

1990a "La structure ternaire du système des déictiques dans les Textes des Sarcophages," Studi di Egittologia e di Antichità Puniche 7, 27-45. 
(Vernus, Pascal)

1990b Future at Issue. Tense, Mood and Aspect in Middle Egyptian: Studies in Syntax and Semantics. Yale Egyptological Studies 4. New Haven.

1994 "Un emploi particulier du démonstratif $n w$ dans les Textes des Sarcophages," in Catherine Berger et al., ed., Hommages à Jean Leclant (4 vols; Bibliothèque d'étude 106; Cairo), IV, 411-15.

1996a "Langue littéraire et diglossie," in Antonio Loprieno, ed., Ancient Egyptian Literature, History and Forms (Probleme der Ägyptologie 10; Leiden and New York), 555-64.

1996b "La position linguistique des Textes des Sarcophages," in Harco Willems, ed., The World of the Coffin Texts (Egyptologische Uitgaven 9; Leuven), 143-96.

1997 Les parties du discours: autopsie d'une théorie. Cahiers de la Société d'égyptologie de Genève 5. Genève.

1998 "La grammaticalisation en égyptien ancien: phrase nominale et morphogenèse de l'inaccompli et du futur," Mémoires de la Société de Linguistique de Paris 5, 63-83.

2000 "Situation de l'égyptien dans les langues du monde," in François-Xavier W. Fauvelle-Aymar et al., ed., Afrocentrisme: l'histoire des Africains entre Égypte et Amérique (Paris), 169-208.

2001 "Le nom d'action et étymologique comme modificateur du verbe: une construction égyptienne proche du maf 'ül mutlaq," in Josep Cervelló Autuori and Alberto J. Quevedo Álvarez, ed., Ir a buscar leña: estudios dedicados al Prof. Jesús López (Aula Aegyptiaca Studia 2; Barcelona), 193-202.

2003 "La piété personnelle à Deir el-Médineh: la construction de l'idée de pardon," in Guillemette Andreu, ed., Deir el-Médineh et la Vallée des Rois: la vie en Egypte au temps des pharaons du Nouvel Empire. Actes du colloque organisé par le Musée du Louvre, les 3 et 4 mai 2002 (Paris), 309-47.

2004 "Le syntagme de quantification en égyptien de la première phase: sur les relations entre Textes des Pyramides et Textes des Sarcophages," in Susanne Bickel and Bernard Mathieu, ed., D'un monde à l'autre: Textes des Pyramides et Textes des Sarcophages, actes de la table ronde internationale "Textes des Pyramides versus Textes des Sarcophages, ” IFAO 24-26 septembre 2001 (Bibliothèque d'étude 139; Cairo), 279-311.

2006 "Pronoms interrogatifs en égyptien de la première phase," Lingua Aegyptia 14, 145-78.

2009 "Le préformant $n$ et la détransitivité Formation $n \mathrm{C}_{1} \mathrm{C}_{2} \mathrm{C}_{1} \mathrm{C}_{2}$ versus $\mathrm{C}_{1} \mathrm{C}_{2} \mathrm{C}_{1} \mathrm{C}_{2}$ : à propos de la racine $\sqrt{g m}$ 'notion de trituration'," Lingua Aegyptia 17, 291-317

2009-10 “Comment l'élite se donne à voir dans le programme décoratif de ses chapelles funéraires: stratégie d'épure, stratégie d'appogiature et le frémissement du littéraire," in Juan C. Moreno García, ed., Élites et pouvoir en Égypte ancienne: actes du colloque Université Charles-de-Gaulle-Lille 3, 7 et 8 juillet 2006 (Cahier de Recherches de l'Institut de Papyrologie et d'Égyptologie de Lille 28; Lille), 67-116.

2010a "Du moyen égyptien au néo-égyptien, de $m$ à $m$-jr: l'auxiliation de l'impératif négatif à la dix-huitième dynastie," in Zahi Hawass and Jennifer H. Wegner, ed., Million of Jubilees: Studies in Honor of David P. Silverman (2 vols.; Annales du Service des Antiquités de l’Égypte, Supplément 39; Cairo), II, 315-35.

2010b Sagesses de l'Égypte pharaonique, 2nd ed. Paris.

2010-11 “'Littérature,' 'littéraire' et supports d'écriture: contribution à une théorie de la littérature dans l'Égypte pharaonique," Egyptian and Egyptological Documents, Archives, Libraries 2, 19-145.

2012 "Le verbe gm(j): essai de sémantique lexicale," in Stéphane Polis and Jean Winand, ed., Lexical Semantics in Ancient Egyptian (Lingua Aegyptia Studia Monographica 9; Hamburg), 387-438.

2013a “The 'Royal Command' (wd-nsw): a Basic Deed of Executive Power," in Juan C. Moreno García, ed., Ancient Egyptian Administration (Handbuch der Orientalistik I, 104; Leiden and Boston), 259-340.

2013b "L'acte fondamental du pouvoir dans l'Égypte pharaonique: l' ordre royal' (oudj nesou)," in Silvia Bussi, ed., Egitto dai Faraoni agli Arabi: atti del convegno Egitto: amministrazione, economia, società, cultura dai faraoni agli arabi, Milano, Università degli studi, 7-9 gennaio 2013 (Pisa and Rome), 21-35. 
2013c "La datation de l'Enseignement d'Aménemopé: le littéraire et le linguistique," in Gerald Moers et al., ed., Dating Egyptian Literary Texts. "Dating Egyptian Literary Texts," Göttingen, 9-12 June 2010, vol. I (Lingua Aegyptia Studia Monographica 11; Hamburg), 191-236.

2014 "Élaboration littéraire et affectation archaïsante: comment Sinohé sait se mettre en avant en se mettant à l'écart," in Harold M. Hays et al., ed., Interpretations of Sinuhe: Inspired by Two Passages (Proceedings of a Workshop held at Leiden University, 27-29 November 2009) (Egyptologische Uitgaven 27; Louvain), 189-213.

to appear a "Autobiographie et scènes dites 'de la vie quotidienne': de la parodie à la fiction du paysan prototypique," in Philippe Collombert, ed., Cinquantenaire de la Mission Archéologique Française de Saqqara.

to appear b "Modelling the Relationship between Reproduction and Production of 'Sacralized' Texts in Pharaonic Egypt," in Todd Gillen, ed., Tradition and Innovation.

von Deines, Hildegard

1954 "Die Demonstrativa im Wundenbuch des Pap. Edwin Smith," Mitteilungen des Instituts für Orientforschung 2, 1-29.

Vycichl, Werner

1955 "Gab es eine Pluralendung -w im Ägyptischen?," Zeitschriften der Deutschen Morgenländischen Gesellschaft $105=$ NF 30, 261-70.

1990 La vocalisation de la langue égyptienne, I: La phonétique. Bibliothèque d'étude 16. Cairo.

Wälchli, Bernhard, and Michael Cysouw

2012 "Lexical Typology through Similarity Semantics: Toward a Semantic Map of Motion Verbs," Linguistics 50, 671-710.

Wb. $\quad$ Adolf Erman and Hermann Grapow, ed. Wörterbuch der ägyptischen Sprache im Auftrage der deutschen Akademien, 9 vols. Berlin, 1971.

Walenta, Edeltraud

1998 "Das Pseudopartizip der Sargtexte als Perfekt und Stativ." Doctoral Dissertation, University of Vienna (unpublished).

Wente, Edward F.

1975-76 "A Misplaced Letter to the Dead," Orientalia Lovaniensia Periodica 6-7, 595-600.

1990 Letters from Ancient Egypt. Writings from the Ancient World 1. Atlanta.

Werning, Daniel

2007 Table "Transkriptionssysteme wichtiger ägyptologischer Werke" in "Ägyptische Hieroglyphen," "Wikipedia, Die freie Enzyklopädie” http://de.wikipedia.org/w/index.php?title=Ägyptische_Hieroglyphen \&oldid=33311049 (18. June 2007, 14:00 UTC; accessed: 27 Jan. 2013).

2008a "Aenigmatische Schreibungen in Unterweltsbüchern des Neuen Reiches: gesicherte Entsprechungen und Ersetzungsprinzipien," in Carsten Peust, ed., Miscellanea in honorem Wolfhart Westendorf (Göttingen), 124-52.

$2008 \mathrm{~b} \quad$ "Aspect vs. Relative Tense, and the Typological Classification of the Ancient Egyptian $s d m . n=f$," Lingua Aegyptia 16, 261-92.

2011 Das Höhlenbuch: textkritische Edition und Textgrammatik, 2 vols. Göttinger Orientforschungen IV, 48. Wiesbaden.

2012 "Ancient Egyptian Prepositions for the Expression of Spatial Relations and Their Translations, a Typological Approach," in Eitan Grossman et al., ed., Lexical Semantics in Ancient Egyptian (Lingua Aegyptia Studia Monographica 9; Hamburg), 293-346. 
(Werning, Daniel)

2013 "Linguistic Dating of the Netherworld Books Attested in the New Kingdom," in Gerald Moers et al., ed., Dating Egyptian Literary Texts. "Dating Egyptian Literary Texts, " Göttingen, 9-12 June 2010, vol. I (Lingua Aegyptia Studia Monographica 11; Hamburg), 237-81.

2014 "Uninflected Relative Verb Forms as Converbs and Verbal Rhemes: the Two Schemes of the Emphatic Construction as a Detached Adjectival Phrase Construction and as a Truncated Balanced Sentence," in Eitan Grossman et al., ed., On Forms and Functions in Ancient Egyptian: Studies in Ancient Egyptian Grammar (Lingua Aegyptia Studia Monographica 15; Hamburg), 309-38.

Westendorf, Wolfhart

1953 "Dynamischer Gebrauch des passivisch-intransitiven Pseudopartizips," Mitteilungen des Instituts für Orientforschung 1, 38-56.

Wierzbicka, Anna

1996 Semantics. Primes and Universals. Oxford.

Wild, Henri

1953 Le tombeau de Ti, II: la chapelle (première partie). Mémoires de l'Institut français d'archéologie orientale 65, 2. Cairo.

Willems, Harco

1990 "Crime, Cult and Capital Punishment (Móalla Inscription 8)," Journal of Egyptian Archaeology 76, 27-54.

Willis, David, Christopher Lucas, and Anne Breitbarth

2013 "Comparing Diachronies of Negation," in David Willis et al., ed., The History of Negation in the Languages of Europe and the Mediterranean, I: Case Studies (Oxford Studies in Diachronic and Historical Linguistics 5; Oxford), 1-50.

Winand, Jean

1991 "Iy et iw: unité morphologique et sémantique," Lingua Aegyptia 1, 357-88.

1992 Études de néo-égyptien 1: La morphologie verbale. Ægyptiaca Leodiensia 2. Liege.

1998 "Prolégomènes à une théorie unifiée de la temporalité en égyptien," in Christopher J. Eyre, ed., Proceedings of the Seventh International Congress of Egyptologists (Orientalia Lovaniensia Analecta 82; Leuven), 1235-45.

1999 "Un dictionnaire des verbes néo-égyptiens," in Stefan Grunert and Ingelore Hagemann, ed., Textcorpus und Wörterbuch: Aspekte zur ägyptischen Lexikographie (Probleme der Ägyptologie 14; Leiden, Boston, and Köln, 1999), 136-49.

2000 "La progression au sein de la narration en égyptien: éléments d'une grammaire du texte," Bulletin de l'Institut Français d'Archéologie Orientale 100, 403-35.

2003 "A Dictionary between Lexicon and Grammar: Interplay of Verbal Aktionsart and Grammatical Aspects," in Ingelore Hafemann, ed., Wege zu einem digitalem Corpus Ägyptischer Texte: Akten der Tagung 'Datenbanken im Verbund' (Berlin, 30. September - 2. Oktober 1999) (Thesaurus Linguae Aegyptiae 2; Berlin), 252-70.

2004 "La non-expression de l'objet direct en égyptien ancien: études valentielles, I," Lingua Aegyptia, 12, 205-234.

2006a Temps et aspect: une approche sémantique. Probleme der Ägyptologie 25. Leiden and Boston.

2006b "A Semantic Approach to the Egyptian Language: the Case of Time and Aspect. Towards a New Paradigm," Lingua Aegyptia 14, 451-472.

2007 "Les formes nominalisées en égyptien ancien," Faits de langue 30, 69-82.

2013 Les hiéroglyphes égyptiens. Que sais-je? Paris.

2015 "Direct Object Marking (DOM) in Ancient Egyptian," in Eitan Grossman et al., ed., Egyptian-Coptic Linguistics in Typological Perspective (Empirical Approaches to Language Typology 55; Berlin), 533-60. 
Woods, Christopher

2008 The Grammar of Perspective: the Sumerian Conjugation Prefixes as a System of Voice. Cuneiform Monographs 32. Leiden and Boston.

Wright, William

1967 A Grammar of the Arabic Language, 3rd ed. Cambridge.

Wüthrich, Annik

2009 "Abracadabras méroïtiques dans le Livre des Morts?," in Burkhard Backes et al., ed., Ausgestattet mit den Schriften des Thot: Festschrift für Irmtraut Munro zu ihrem 65. Geburtstag (Studien zum Altägyptischen Totenbuch 14;Wiesbaden), 267-82.

Zakrzewska, Ewa D

1990 "Syntactic Characteristics of the Old Perfective in the Coffin Texts," Rocznik Orientalistyczny 47, 123-45.

Zanuttini, Raffaella

1997 Negation and Clausal Structure: a Comparative Study of Romance Languages. Oxford Studies in Comparative Syntax. Oxford.

Zeidler, Jürgen

1992 Review of Karel Petráček, Vergleichende Studien, Lingua Aegyptia 2, 189-222.

1999 Pfortenbuchstudien, 2 vols. Göttinger Orientforschungen IV, 36. Wiesbaden.

Zonhoven, Louis

1996 "Studies on the $s \underline{d}$ m.t $=f$ Verb Form in Classical Egyptian I: the Construction $\underline{d} r \underline{d} \underline{d} . t=f$," Bibliotheca Orientalis 3, $614-43$.

1997a "Studies on the $s \underline{d}$ m.t $f$ Verb Form in Classical Egyptian II: the Active $r$ s $\underline{d} m . t=f$ Construction," Orientalia Lovaniensia Periodica 28, 5-31.

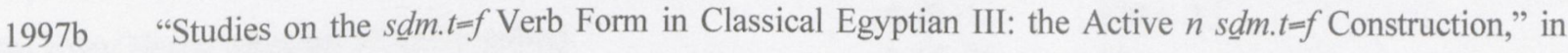
Jcabus van Dijk, ed., Essays on Ancient Egypt in Honour of Herman Te Velde (Egyptological Memoirs 1; Groeningen), 383-400. 